\title{
Nationales Reformprogramm Österreich
}

Bundeskanzleramt 
Wien, April 2012 


\section{Inhalt}

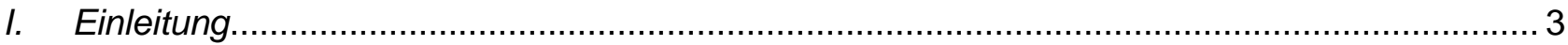

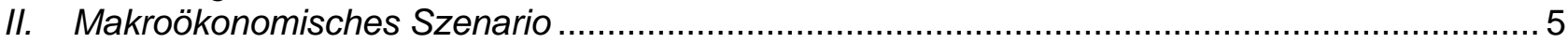

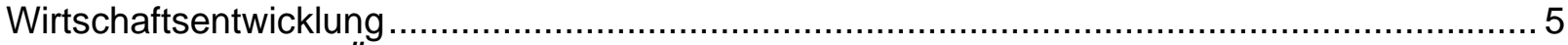

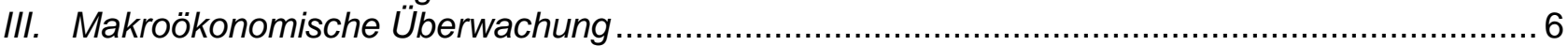

Stabilität, Qualität und Nachhaltigkeit in den öffentlichen Finanzen...................................... 6

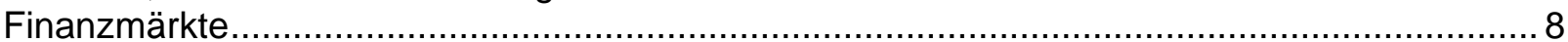

Wettbewerbsfähigkeit und Auslandsverschuldung ........................................................ 10

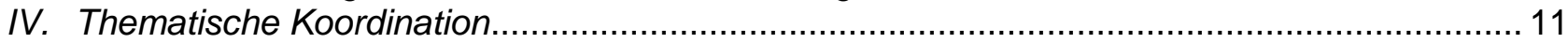

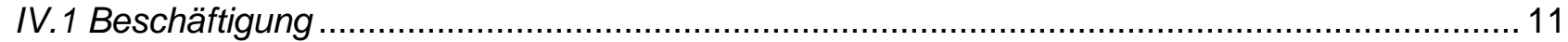

Arbeitsmarktbeteiligung älterer Arbeitnehmerinnen und Arbeitnehmer................................ 12

Steigerung der Erwerbsbeteiligung bestimmter Zielgruppen ........................................... 15

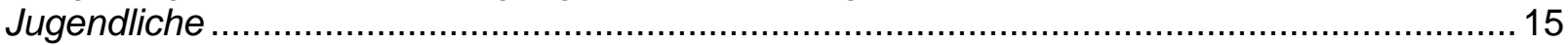

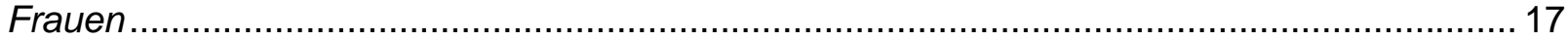

Personen mit Migrationshintergrund ……………………………………………….... 20

Reduzierung der Steuer- und Abgabenlast von Arbeitnehmerlnnen..................................... 21

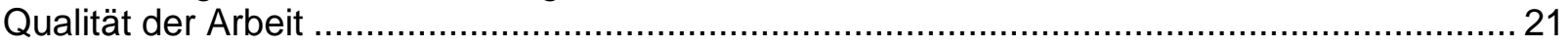

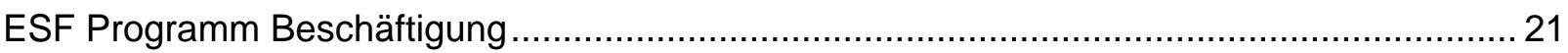

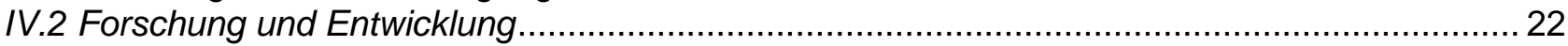

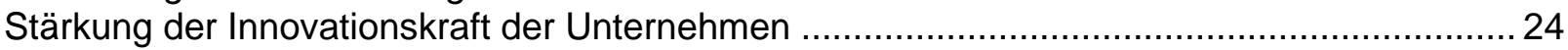

Strategische Ausrichtung und Umsetzung bestehender Maßnahmen ...................................26

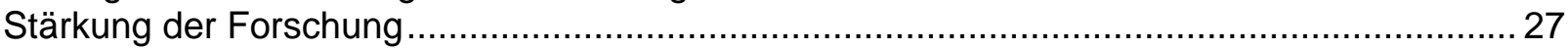

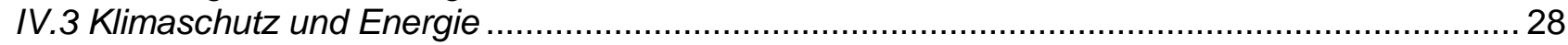

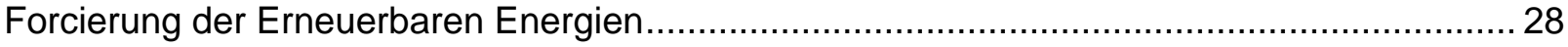

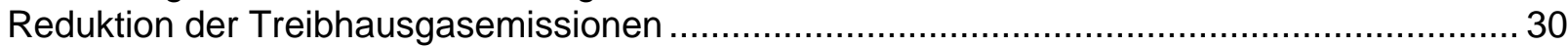

Erhöhung der Energieeffizienz ………………………………………………………. 30

Effizienter Umgang mit natürlichen Ressourcen - Ressourceneffizienz................................. 32

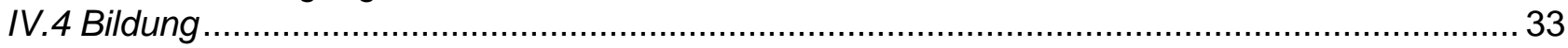

Steigerung der Bildungsbeteiligung, Vorbereitung auf das Studium und Erhöhung der

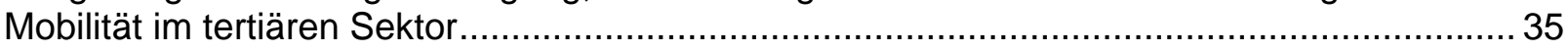

Anzahl der Absolventinnen und Absolventen naturwissenschaftlich-technischer

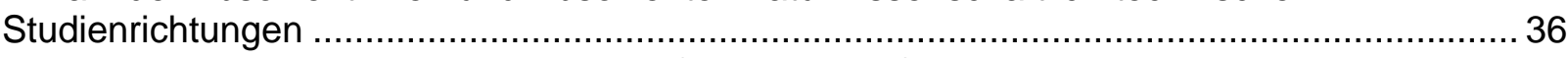

Verbesserung des Bildungsniveaus und Senkung der Schulabbrecherinnen- und

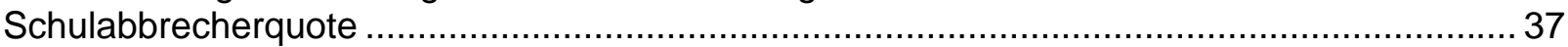

Attraktivität, Qualität und Durchlässigkeit der beruflichen Bildung erhöhen .............................. 40

IV.5 Verminderung von Armut und sozialer Ausgrenzung ....................................................... 40

Bekämpfung der Langzeitarbeitslosigkeit durch eine Verbesserung der

Erwerbsbeteiligung armuts- und ausgrenzungsgefährdeter Gruppen im erwerbsfähigen

Alter

Maßnahmen zur Gesundheitsprävention am Arbeitsplatz und verstärkte

Arbeitsmarkt(re)integration von Menschen mit gesundheitlichen Einschränkungen und

Menschen mit Behinderungen.

Reduktion der frauenspezifischen Benachteiligungen in den Bereichen Einkommen und

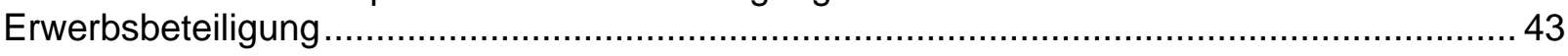

Bekämpfung der Kinder- und Jugendarmut und der Vererbung von Armut ......................... 44

IV.6 Wettbewerb und unternehmerisches Umfeld ………….................................................. 45

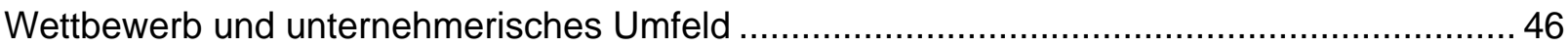

Förderung von Unternehmertum / Gründungdynamik..................................................... 47 
Annex 1: Beiträge der Sozialpartner, Länder und Gemeinden.

Annex 2: Reporting table on national Europe 2020 targets and other key commitments

Table 1. Standard table for the assessment of CSRs and key macro-structural reforms in the NRPS 


\section{Einleitung}

I.1 Das Europäische Semester 2012 wendet erstmals die verstärkte wirtschaftspolitische Steuerung an, wie sie im Rahmen des sogenannten „Six-Packs“ vereinbart wurde. Dieses verpflichtet die Mitgliedstaaten, ihre jährliche Haushaltsplanung stärker mit der Europäischen Kommission abzustimmen. Darüber hinaus sind in den Nationalen Reformprogrammen die Mitgliedstaaten dazu aufgefordert, die vom Europäischen Rat ${ }^{1}$ auf seiner Märztagung bestätigten Reformprioritäten des Jahreswachstumsberichts $2012^{2}$ und des Gemeinsamen Beschäftigungsberichts ${ }^{3}$ umzusetzen.

I.2 Es gilt festzuhalten, dass die fünf nationalen Europa 2020 Ziele nach wie vor uneingeschränkt gültig sind, ebenso die - im Zuge der nationalen Zieldefinition für jedes Kernziel identifizierten wichtigsten - Wachstumshemmnisse. Die Ratsempfehlungen werden in der Politikgestaltung berücksichtigt und spiegeln in wesentlichen Bereichen auch die nationalen Schwerpunkte wider, um ein intelligentes, nachhaltiges und teilhabeförderndes Wachstum, Wettbewerbsfähigkeit, Beschäftigung und sozialen Zusammenhalt zu stärken und gleichzeitig die Klima- und Energieziele zu erreichen.

I.3 Der Euro-Plus-Pakt dient insbesondere dem Ziel die österreichische Wettbewerbsfähigkeit zu stärken. Inhaltlich tragen die Maßnahmen dazu bei, die Ziele der Europa 2020 Strategie zu erreichen.

I.4 Die Staats- und Regierungschefs haben bei ihrer Zusammenkunft zum informellen Europäischen Rat Ende Jänner 2012 vereinbart, mehr Gewicht auf beschäftigungspolitische Impulse, insbesondere für junge Menschen, zu legen. Und die Mitgliedstaaten wurden aufgefordert, die konkreten Maßnahmen in „,nationalen Beschäftigungsplänen“ darzulegen. Die österreichische Bundesregierung hat bereits im Rahmen des Euro-Plus-Pakts einen beschäftigungspolitischen Schwerpunkt gesetzt. Im nationalen Kontext wird der Arbeitsmarkt- und Beschäftigungspolitik für Jugendliche im Jahr 2012 höchste Priorität eingeräumt.

I.5 Die Österreichische Bundesregierung ist bemüht, die Europa 2020 Strategie in enger Einbindung der Länder, Regionen und Gemeinden sowie aller relevanten Interessenvertreter als einen breit angelegten Beteiligungsprozess umzusetzen.

I.6 Die Österreichischen Sozialpartner ${ }^{4}$ haben ihre Stellungnahmen zum Nationalen Reformprogramm übermittelt und eine Dokumentation einschlägiger Aktivitäten zur Umsetzung von Europa 2020 zur Verfügung gestellt.

I.7 Auch die Bundesländer, Städte und Gemeinden haben aus ihren vielfältigen Maßnahmen, die die Erreichung der nationalen Europa 2020 Ziele unterstützen, jeweils ein bis zwei Vorzeigeprojekte ausgewählt. Diese Dokumentation kann in einem eigenen Anhang eingesehen werden.

I.8 Nichtregierungsorganisationen werden über ihre zivilgesellschaftlichen Dachverbände im Wege der jeweils federführenden Ressorts eingebunden. Im Bereich der Armutsbekämpfung

\footnotetext{
${ }^{1}$ Europäischer Rat (2012), Tagung des Europäischen Rates 1./2. März 2012, Schlussfolgerungen (EUCO4/12);

2 Europäische Kommission (2012), Mitteilung der Kommission. Jahreswachstumsbericht 2012 (KOM(2011) 815 endg.)

${ }^{3}$ Gemeinsamer Beschäftigungsbericht 2012 (5899/2/12)

${ }^{4}$ siehe Annex 1 und nähere Informationen zu den österreichischen Sozialpartnern unter http://www.sozialpartner.at
} 
beispielsweise hat sich im Kontext der Offenen Methode der Koordinierung (OMK) ein Informations- und Konsultationsmechanismus entwickelt, der sich um ein hohes Beteiligungsausmaß bemüht. Ähnlich dichte Kooperationsprozesse existieren im Bereich der Strukturpolitik und auch in der Klima- und Umweltpolitik, bei der Überarbeitung der nationalen Nachhaltigkeitsstrategie oder etwa im Rahmen der Stakeholder-Initiative „Wachstum im Wandel“.

I.9 Im Anschluss an die Übermittlung des Nationalen Reformprogramms im April 2011, welche gemeinsam mit dem Stabilitätsprogramm erfolgte, nahm die Europäische Kommission eine integrierte Beurteilung vor und formulierte Vorschläge für spezifische Empfehlungen für Österreich. Nach eingehenden Diskussionen dieser Empfehlungen im Wirtschafts- und Finanzausschuss, Wirtschaftspolitischen Ausschuss und Beschäftigungsausschuss verabschiedeten die jeweils zuständigen Fachministerräte ECOFIN und BESOGEKO - die Länderspezifischen Empfehlungen des Jahres 2011, die dem Europäischen Rat im Juni ${ }^{5}$ zur Bestätigung vorgelegt wurden. Die formelle Verabschiedung der Empfehlungen erfolgte im Juli durch den Rat in seinen Formationen ECOFIN und BESOGEKO.

I.10 Diese Ratsempfehlungen sind Teil der österreichischen Reformagenda 2012. In den jeweiligen Kapiteln zu den sechs Reformbereichen wird über die Fortschritte und Umsetzung der Maßnahmen und der länderspezifischen Empfehlungen vom Juni 2011 berichtet sowie eine Bestandsaufnahme der Euro-Plus-Pakt Maßnahmen vorgenommen.

I.11 Die länderspezifischen Empfehlungen ${ }^{6}$ im Wortlaut:

Der Rat empfiehlt, dass Österreich im Zeitraum 2011-2012 Maßnahmen ergreift, um:

1. die derzeitige Konjunkturerholung zu nutzen, um die Korrektur des vor allem auf der Ausgabenseite geplanten übermäßigen Defizits zu beschleunigen und dadurch den hohen öffentlichen Schuldenstand auf einen Abwärtspfad zu bringen; zu diesem Zweck, einschließlich auf subnationaler Ebene, die erforderlichen Maßnahmen anzunehmen und umzusetzen, um für den Zeitraum 2011 bis 2013 in Übereinstimmung mit den Empfehlungen des Rates im Rahmen des Verfahrens bei einem übermäßigen Defizit eine jährliche Konsolidierungsanstrengung von durchschnittlich $0,75 \%$ des BIP zu gewährleisten; nach der Korrektur des übermäßigen Defizits die Maßnahmen anzugeben, die erforderlich sind, um im Einklang mit dem Stabilitäts- und Wachstumspakt (SWP) einen angemessenen Fortschritt hinsichtlich des mittelfristigen Ziels zu gewährleisten.

2. Schritte zu unternehmen, um den nationalen Budgetrahmen durch eine Angleichung der legislativen und administrativen Zuständigkeitsbereiche sowie der Zuständigkeiten für die Einnahmenerhebung und die Ausgabenbefugnis zwischen den verschiedenen Regierungsebenen zu stärken, insbesondere im Bereich des Gesundheitswesens.

3. in Abstimmung mit den Sozialpartnern und gemäß den nationalen Praktiken Schritte einzuleiten, um den Zugang zur derzeitigen Frühpensionsregelung für Langzeitversicherte weiter $\mathrm{zu}$ begrenzen und Schritte einzuleiten, um die Übergangszeit für die Harmonisierung des gesetzlichen Pensionsantrittsalters für Frauen und Männer zu verkürzen, damit die langfristige Tragfähigkeit und die Angemessenheit des Pensionssystems sichergestellt werden kann; die Voraussetzungen für den Zugang zur Invaliditätspension streng anzuwenden.

4. die Erwerbsbeteiligung zu steigern, wozu auch gehört, dass es die effektive Steuerund Sozialversicherungsbelastung der Arbeit, insbesondere für Niedrig- und

\footnotetext{
${ }^{5}$ Europäischer Rat (2011), Schlussfolgerungen des Europäischen Rates 23./24. Juni 2011 (EUCO 23/1/11rev) http://www.consilium.europa.eu/uedocs/cms data/docs/pressdata/de/ec/123098.pdf

${ }^{6}$ Empfehlungen des Rates vom 12. Juli 2011 zum nationalen Reformprogramm Österreichs 2011 und Stellungnahme des Rates zum aktualisierten Stabilitätsprogramm Österreichs für 2011-2014, Brüssel 2011 (http://eur-lex.europa.eu/LexUriServ/LexUriServ.do?uri=OJ:C:2011:210:0008:0011:DE:PDF)
} 
Durchschnittslohnempfänger, budgetneutral reduziert; den nationalen Aktionsplan zur Gleichbehandlung von Frauen und Männern auf dem Arbeitsmarkt umzusetzen, einschließlich Verbesserungen in Bezug auf das Angebot an Ganztagesbetreuungseinrichtungen und Pflegediensten, um die Möglichkeiten für ganztags arbeitende Frauen auszuweiten und den hohen geschlechtsspezifischen Lohnunterschied zu reduzieren; weitere Schritte zu unternehmen, um die Ergebnisse im Bildungsbereich zu verbessern und Schulabbrüche zu verhindern.

5. weitere Schritte zur Förderung des Wettbewerbs zu unternehmen, insbesondere im Dienstleistungssektor, indem die Markteintrittsschranken gelockert, ungerechtfertigte Beschränkungen von Gewerbe und Berufen beseitigt sowie die Befugnisse der Wettbewerbsbehörden gestärkt werden; die Annahme der ausstehenden „horizontalen Rechtsvorschriften“ zur Umsetzung der Dienstleistungsrichtlinie zu beschleunigen.

\section{Makroökonomisches Szenario}

Siehe Österreichisches Stabilitätsprogramm für die Jahre 2011 bis 2016

\section{Wirtschaftsentwicklung}

II.1 Die österreichische Wirtschaft ist im Jahr 2011 kräftig gewachsen. Das reale BIP legte um 3,1\% gegenüber dem Vorjahr zu (nach 2,3\% im Jahr 2010) - höhere Wachstumsraten wurden im abgelaufenen Jahrzehnt nur in der Hochkonjunkturphase 2006-2007 erreicht. Die gute Wachstumsperformance ist auf einen deutlichen Wachstumsüberhang aus dem Jahr 2010 und auf das sehr starke erste Halbjahr 2011 zurückzuführen. Hohe Exportzuwächse im Gefolge des kräftigen Aufwinds der Weltwirtschaft und Aufholbedarf bei Unternehmensinvestitionen beflügelten die Konjunktur. Im zweiten Halbjahr ließ die Exportdynamik spürbar nach, stabilisierend wirkten Ausrüstungsinvestitionen und privater Konsum.

II.2 Fehlende Impulse vom Außenhandel dürften auch im ersten Halbjahr 2012 das Wachstum lähmen. Ein Ende der globalen Abkühlung ist jedoch in Sicht. Die meisten nationalen und internationalen Frühindikatoren erreichten im November 2011 einen Tiefpunkt und verbesserten sich seither kontinuierlich. Auch in Österreich dürfte die Bodenbildung bei den Erwartungen zu Jahresende erreicht worden sein; wichtige Vorlaufindikatoren deuten seither kontinuierlich nach oben und signalisieren einen moderaten Aufschwung ab Jahresmitte. Die Prognosen für Österreich für das Jahr 2012 reichen von einer Wachstumsrate des realen BIP von $+0,4 \%$ (WIFO März 2011) bis $+0,7 \%$ (EK Feb. 2012) im heurigen Jahr.

II.3 Die Konjunktureinschätzung verbesserte sich insbesondere in der Sachgütererzeugung. 2011 gab es einen Exportrekord in der Höhe von 122,1 Mrd. € an Ausfuhren (im Vergleich: bisheriger Rekord 2008 bis 117,5 Mrd. €) und auch die Aussichten im Investitions- und Konsumgüterbereich sind durchwegs positiv. Die Bereitschaft der Unternehmen zu Erweiterungsinvestitionen wächst angesichts der verbesserten Stimmungslage und der zuletzt merklich höheren Kapazitätsauslastung.

II.4 Gestützt wird die Entwicklung auch von einem stetigen Zuwachs des privaten Konsums, der auch im zweiten Halbjahr 2011 mit zunehmenden Raten expandierte. Für das Jahr 2012 können die Arbeitnehmerinnen und Arbeitnehmer dank sinkender Teuerungsraten (von zuletzt deutlich über 3\%) mit Reallohnzuwächsen rechnen. Die Lohn- und Gehaltssumme steigt 2012 um 4,1\% gemäß WIFO, wodurch bei einem erwarteten Rückgang der Teuerungsrate heuer auf 2,5\% (HVPI) im Vergleich zum Vorjahr eine Stärkung der Kaufkraft und eine robuste Inlandsnachfrage zu erwarten ist. Dazu prognostiziert das WIFO eine leichte Verringerung der Sparquote dem Konjunkturzyklus folgend von 7,5\% auf 7,3\%. 
II.5 Der Arbeitsmarkt entwickelte sich 2011 ausgesprochen gut. Die Zahl der aktiv Erwerbstätigen stieg im Jahr 2011 um etwa 71.000 (+1,9\%) auf rund 3.757.000 Personen und lag damit über der Zahl der Erwerbstätigen vor der Wirtschaftskrise. Bei einem Rückgang um etwa 4.000 Personen gegenüber 2010 lag die Zahl der arbeitslos gemeldeten Personen mit rund 247.000 in 2011 um 35.000 höher als vor der Krise (2008). Die Arbeitslosenquote ging 2011 gegenüber dem Vorjahr um 0,2 Prozentpunkte auf 4,2\% zurück und erreichte den niedrigsten Stand in der gesamten EU, wo der Durchschnitt nahe bei $10 \%$ liegt. Im Jahr 2012 erwartet das WIFO einen leichten Anstieg der Arbeitslosenquote in Österreich. Der gesamtwirtschaftliche Produktivitätsfortschritt betrug 2010 und 2011 jeweils rund 1,5\%. Aufgrund der Konjunktureintrübung wird 2012 mit einer leicht negativen Produktivitätsentwicklung gerechnet, da Unternehmen kaum Arbeitsplätze abbauen.

\section{Makroökonomische Überwachung}

III.1 Basierend auf der Governance-Struktur der Europa 2020-Strategie und den Vorgaben zur Erstellung der Nationalen Reformprogramme werden im folgenden Abschnitt für die makroökonomische Überwachung relevante Informationen im Lichte der horizontalen Politikorientierung durch den Europäischen Rat, der integrierten Leitlinien, der länderspezifischen Empfehlungen an Österreich des Jahres 2011 sowie den Analyseergebnissen des „Frühwarnberichts“ 2012 der Europäischen Kommission im Rahmen des Verfahrens bei Makroungleichgewichten dargestellt.

\section{Stabilität, Qualität und Nachhaltigkeit in den öffentlichen Finanzen}

III.2 Die Wirtschafts- und Finanzkrise und die notwendige wirtschaftspolitische Reaktion mittels umfangreicher konjunkturstabilisierender Maßnahmen hatten den bereits eingeschlagenen Kurs der Sicherstellung der finanziellen Nachhaltigkeit und der über den Konjunkturzyklus anvisierten Ausgeglichenheit des gesamtstaatlichen Haushalts in Österreich stark unter Druck gesetzt. Um frühzeitig mit der einsetzenden Konjunkturerholung dem Anstieg der Schuldenquote gegenzusteuern, wurde schon mit dem Budget 2011 ein ausgabenseitig orientiertes Bündel an Konsolidierungsmaßnahmen in der Höhe von kumuliert rd. 13,6 Mrd. Euro (2011-2014) beschlossen (Details siehe NRP 2011) und bis dato konsequent umgesetzt. Die im Zuge der Steuerreform 2009 umgesetzte Absenkung der Tarife wurde zudem bewusst nicht zurückgenommen. Auch der überwiegende Teil der Erleichterungen für Familien wurde beibehalten. Diese Maßnahmen leisten somit weiterhin einen wichtigen Beitrag zur steuerlichen Entlastung des Faktors Arbeit.

III.3 Das Jahr 2011 stand daher wesentlich im Zeichen einer starken Rückführung des gesamtstaatlichen Budgetdefizits. Aufgrund einer straffen Umsetzung des ersten Konsolidierungspakets, einer guten Konjunkturlage und der Sicherstellung von Ausgabendisziplin konnte dieses deutlich von 4,4\% (2010) auf 2,6\% des BIP (2011) reduziert werden. Die budgetären Gesamtausgaben lagen dabei 2011 unter und die Gesamteinnahmen über den Werten, welche für dieses Jahr vorveranschlagt waren. Für heuer ist ein Defizitwert von 3\% des BIP vorgesehen. Dieser „rebound“ ist in erster Linie auf die schlechtere Konjunkturlage sowie auf eine zeitlich verschobene Buchung von bestimmten Ausgabenströmen ${ }^{7}$ zurückzuführen Österreich bekennt sich zu den Vorgaben im Rahmen des aktuellen Verfahrens wegen eines übermäßigen Defizits nach Art. 126 AEUV und plant sein übermäßiges Budgetdefizit bis spätestens $2013 \mathrm{zu}$ korrigieren. Insgesamt ist vorgesehen, im Jahr 2013 ein gesamtstaatliches Defizit von 2,1\% des BIP sowie eine Schuldenquote von $75,3 \%$ des BIP zu realisieren. Weiters wird das gesamtstaatliche strukturelle Defizit bis 2016 im Einklang mit den neuen Bestimmungen des

\footnotetext{
${ }^{7}$ Details siehe Stabilitätsprogramm für die Jahre 2011 bis 2016
} 
„Six-Packs" und des „Vertrags über Stabilität, Koordinierung und Steuerung in der Wirtschafts - und Währungsunion" (VSKS) auf 0,4\% des BIP rückgeführt. Die Schuldenquote soll bis 2016 auf rund $70 \%$ des BIP zurückgehen. In diesem Sinne wurde der Konsolidierungspfad im Budget 2012 fortgeführt, mit dem Stabilisierungspaket 2012 wurden im Frühjahr zusätzliche Reformschritte mit einem Gesamtvolumen von rd. 27,8 Mrd. Euro (2012-2016) beschlossen. Gesamtstaatlich werden zwei Drittel aus Reduktion von Ausgaben und ein Drittel aus Erhöhung von Einnahmen finanziert. Im Steuerbereich werden vor allem Steuerlücken geschlossen und keine Erhöhung von Massensteuern vorgenommen (Details zu Defizit, Schulden, Ausgaben und Einnahmen siehe Stabilitätsprogramm für die Jahre 2011 bis 2016).

III.4 Neben einer notwendigen ambitionierten Konsolidierung wird - entsprechend der horizontalen Orientierung durch den Europäischen Rat - auch dem Ausbau des Wachstumspotentials Rechnung getragen. Im Rahmen der Maßnahmen zur Realisierung der Europa 2020 Ziele leisten dazu die fortgeführten budgetären Offensivmaßnahmen mit jährlich rund 400 Mio. Euro für die Bereiche Bildung, F\&E und Ressourceneffizienz einen wichtigen Beitrag. Für Universitäten werden zudem ab 2013 zusätzlich 250 Mio. Euro pro Jahr und für Schulen ab 2012 im Schnitt rund 300 Mio. Euro zusätzlich pro Jahr zur Verfügung gestellt.

III.5 Die straffe Umsetzung der Haushaltsrechtsreform in ihren zwei Schritten 2009 und 2013 hat bereits mit ihrer Hauptelementen wie dem bindenden mehrjährigen Ausgabenrahmen, Flexiklauseln und der Performance-Orientierung den Grundstein für die langfristige Nachhaltigkeit der öffentlichen Haushalte in Österreich gelegt. Der Beschluss der "Österreichischen Schuldenbremse“ im Parlament vom 7. Dezember 2011 führt diese stabilitätsorientierte Ausrichtung konsequent fort. Mit diesem Konzept wird der strukturelle Budgetsaldo zur neuen Zielgröße der Wirtschaftspolitik. Bei Abweichungen sind zudem rasch greifende Korrekturmechanismen vorgesehen. Dabei werden Abweichungen auf einem Kontrollkonto erfasst und müssen bei Überschreiten eines Schwellenwertes konjunkturgerecht zurückgeführt werden. Durch diesen Ansatz wird gewährleistet, dass Schulden in konjunkturellen Aufschwungsphasen abgebaut werden und die stabilisierende Funktion öffentlicher Finanzen im Konjunkturabschwung gewahrt wird. Zudem stellt eine präzisere Zuweisung von budgetärer Verantwortung an alle Regierungsebenen eine gesamtstaatliche Orientierung zur budgetären Zielsetzung sicher. Als weitere Stütze ist im Gesetzestext explizit festgeschrieben, dass Rechtsakte der Europäischen Union zur Einhaltung der Haushaltsdisziplin erfüllt werden müssen. Die Schuldenbremse entspricht mit einem vorgeschriebenen strukturellen Defizit des Bundes von maximal $-0,35 \%$ des BIP im Jahr 2017 einer ambitionierten Umsetzung der Vorgaben des europäischen Fiskalpakts.

III.6 Der Österreichische Stabilitätspakt (ÖStP) 2011 ist rückwirkend mit 1.1.2011 in Kraft getreten und hat u.a. ${ }^{8}$ auch eine „Rendez-vous-Klausel“ bei Änderung von EU-rechtlichen Vorgaben (Verhandlungen zur Anpassung) vorgesehen. Aufgrund der verstärkten wirtschaftlichen Governance der EU-Mitgliedstaaten ergibt sich bereits jetzt die Notwendigkeit, Verhandlungen zur Anpassung des ÖStP 2011, der bis 2014 abgeschlossen wurde, zu führen und durch strengere Ziele als bisher die Umsetzung des neuen Konsolidierungspfades und damit die Erreichung eines strukturell ausgeglichenen Haushaltes für ganz Österreich ab 2017 sicherzustellen. Der Österreichische Stabilitätspakt ist insbesondere auch Anker für die Umsetzung einer „Schuldenbremse“ für Bund, Länder und Gemeinden. Mit den Ländern und Gemeinden wurde dazu am 29. November 2011 in

\footnotetext{
${ }^{8}$ Der ÖStP 2011 hat eine Reihe an Neuerungen gebracht: ambitionierte, realistische Stabilitätsbeiträge von Bund, Ländern und Gemeinden, Verschärfungen der Sanktionen bei Zielverfehlung, Verbesserungen der Haushaltskoordinierung und mittelfristigen Ausrichtung der Haushaltsführung (die erste Datenlieferung auf Basis der neuen Formulare erfolgt erstmals im Juni 2012), die Festlegung von autonomen Haftungsobergrenzen für Bund, Länder und Gemeinden, erhöhte Transparenz und eine „Rendez-vous-Klausel“ bei Änderung von EU-rechtlichen Vorgaben (Verhandlungen zur Anpassung).
} 
Salzburg eine Einigung über wesentliche Inhalte erzielt, die nun im Detail ausverhandelt werden.

III.7 Die Nachhaltigkeit in den öffentlichen Finanzen Österreichs wird vor allem über die rasche Implementierung der gesamtstaatlichen „Schuldenbremse“ sichergestellt. Dies erhöht auch künftige Spielräume in den öffentlichen Finanzen. Gleichzeitig gilt es aber, die Wachstumskräfte und Wettbewerbsfähigkeit zu stärken und schon heute Ausgabendynamiken in den Bereichen Pensions-, Gesundheits- und Alterspflege, welche vor allem künftig durch die kommende Alterung der Bevölkerung zu erwarten sind, einzubremsen. Aus diesem Grund werden ein hohes Beschäftigungsniveau älterer ArbeitnehmerInnen sowie die rasche Annäherung des faktischen Pensionsantrittsalters an das gesetzlich vorgegebene und damit eine geringere Anzahl an Frühpensionierungen und Invaliditätspensionen mit Nachdruck verfolgt. Neben einer Reihe von in dieser Richtung wirkenden Reformen der letzten Jahre (siehe NRP 2011) wurde auch im jüngsten Stabilisierungspaket ein besonderes Augenmerk auf diese Aspekte gelegt. Dies umfasst u.a. die Anhebung der Anspruchsvoraussetzungen und Abschläge in der Korridorpension und der vorzeitigen Alterspension bei langer Versicherungsdauer oder die schrittweise Anhebung des Lebensalters, ab welchem der Tätigkeitsschutz greift und damit ein Übertritt in die Invaliditätspension möglich wird, auf 60 Jahre. $^{9}$ Zudem wird im Gesundheitsbereich ein Kostendämpfungspfad umgesetzt, welcher die Ausgabensteigerungen bis 2016 auf 3,6\% jährlich begrenzen soll.

III.8 Ein effektiver und effizienter öffentlicher Sektor, vor allem auch über einen größtmöglichen Einsatz von e-government Lösungen, ist seit längerer Zeit ein wesentliches Reformziel in Österreich. Erfolgreiche Instrumente wie ein Unternehmensserviceportal als one-stop-shop für gesetzliche Meldeverpflichtungen, Finanzonline als intuitive Plattform für die Behördenkommunikation in Steuerangelegenheiten oder die Initiative zur Senkung der Verwaltungslasten für Bürger und Unternehmen unterstreichen diese Ambitionen. Eine weitere umfangreiche Verwaltungsreform wird in den nächsten Jahren Schritt für Schritt umgesetzt. Gestraffte Aufgaben- und Kompetenzverteilungen konnten bereits im Pflegebereich sowie der Verwaltungsgerichtsbarkeit realisiert werden. Ein zwischen Bund, Ländern und Sozialversicherung gemeinsam vereinbartes Zielsteuerungssystem für eine integrative Gesundheitsplanung soll zu signifikanten Einsparungen in der Sozialversicherung von rd. 1,4 Mrd. Euro (2012-2016) und zu einer besseren Aufteilung der budgetären Verantwortung führen. Auch im Pensionsbereich wurde die Verwaltungsreform vorangetrieben: Um eine Invaliditätspension zu erlangen, ist beispielsweise ein ärztliches Gutachten über die Arbeitsfähigkeit der Antragssteller nötig, diese Begutachtung wird künftig vereinheitlicht und zentralisiert erfolgen.

\section{Finanzmärkte}

III.9 Die Schuldenkrise in der Eurozone beeinflusste den Geschäftsverlauf der österreichischen Banken im Jahr 2011 deutlich.Im Vergleich zum Vorjahr reduzierte sich das unkonsolidierte Betriebsergebnis um 7,9\% auf 7,5 Mrd. Euro. U.a. aufgrund höherer Risikovorsorgen ist für 2011 mit einer konsolidierten Gesamtkapitalrentabilität (RoA) des österreichischen Bankensektors von rund 0,13\% zu rechnen. Der Ausstieg aus den staatlichen Hilfsmaßnahmen für den Bankensektor wird bereits umgesetzt. Mit 15. Februar 2012 beliefen sich die Garantien auf Wertpapieremissionen von Finanzinstitutionen noch auf 12 Mrd. Euro, das von fünf österreichischen Bankengruppen gezeichnete Partizipationskapital hatte ein Volumen von 4,8 Mrd. Euro. Da sich die österreichischen Banken stark auf die CESEE Region konzentrieren, sind sie auf den derzeit schwierigen

\footnotetext{
${ }^{9}$ Erste Schätzungen zeigen, dass durch das Stabilitätspaket 2012-2016 das durchschnittliche Pensionsantrittsalter bis zum Jahr 2017 um bis zu 6 Monate ansteigen wird. Eine weitere Erhöhung des effektiven Pensionsantrittsalters soll durch die geplanten Maßnahmen im Bereich der „Invaliditätspensionen unter 50“, die bis Ende 2012 finalisiert sein sollen, erreicht werden.
} 
Märkten Irland, Spanien, Griechenland und Portugal kaum exponiert. Die konsolidierten Auslandsforderungen der mehrheitlich im Inlandsbesitz befindlichen österreichischen Banken gegenüber CESEE-Ländern betrugen Ende Dezember 2011 etwa 220 Mrd. Euro und haben sich somit im vergangenen Jahr etwa auf diesem Niveau stabilisiert. Die CESEEAuslandsforderungen aller österreichischen Banken lagen zeitgleich bei rd. 330 Mrd. Euro. Etwa 70\% der gesamten Auslandsforderungen gegenüber dieser Region bestehen dabei gegenüber EU-Mitgliedsstaaten. Das Exposure in CESEE ist regional stark diversifiziert, womit die Gefahr einer länderspezifischen oder subregionalen Klumpenbildung überschaubar ist.

III.10 Die Finanzmarktaufsichtsbehörde (FMA) und die Oesterreichische Nationalbank (OeNB) haben am 14. März 2012 die „Leitlinie zur Stärkung der Nachhaltigkeit der Geschäftsmodelle international aktiver österreichischer Großbanken" veröffentlicht. Dieses gemeinsam mit der Oesterreichischen Nationalbank (OeNB) erarbeitete Maßnahmenpaket zielt darauf $a b$, die Eigenkapitalbasis dieser Bankengruppen mittel- und langfristig zu erhöhen und die Refinanzierungsstruktur exponierter Tochterbanken ausgewogener zu gestalten. Zudem soll sichergestellt werden, dass die Banken künftig für etwaige Krisensituationen über adäquate Sanierungs- und Abwicklungspläne verfügen. Aufgrund ihrer Größe, systemischen Relevanz und der Komplexität ihrer Geschäftsmodelle (mit einer Vielzahl von Tochterbanken) ist diese Leitlinie derzeit auf drei österreichische Bankengruppen anzuwenden. Die Leitlinie sieht folgende Eckpunkte vor:

- Um die Eigenkapitalbasis nachhaltig zu stärken, sind die neuen Regeln für hartes Kernkapital (CET1) unter Basel III ohne Übergangsfristen bereits mit 1. Jänner 2013 zu erfüllen. Das im Rahmen des Bankenhilfspakets gezeichnete, voll verlusttragfähige, staatliche und private Partizipationskapital wird aber im Rahmen der Übergangsvorschriften angerechnet. Zudem ist ab 1. Jänner 2016 ein zusätzlicher harter Kernkapitalpuffer - je nach Risikogehalt des Geschäftsmodells von bis zu 3 Prozentpunkten zu halten.

- Um die Refinanzierungsstruktur der Tochterbanken zu stärken und auf ihre ausgewogene Gestaltung zu achten, wird die Aufsicht die Nettoneukreditvergabe der Tochterbanken im Verhältnis zur Aufbringung lokaler stabiler Refinanzierungen ${ }^{10}$ laufend beobachten und analysieren. Das Monitoring wird regelmäßig mit Quartalsdaten erfolgen. Die Ergebnisse und Analysen werden im Rahmen der Aufsichtskollegien evaluiert und diskutiert, um gegebenenfalls Aufsichtsmaßnahmen zu ergreifen.

- Damit ein Institut im Krisenfall rasch, effektiv und effizient saniert oder eine eventuelle Insolvenz geordnet ablaufen kann, haben die Mutterinstitute bis Ende 2012 der Aufsicht als Vorsorge für die Bewältigung etwaiger Krisen angemessene Sanierungsund Abwicklungspläne vorzulegen.

III.11 Bei den im Frühjahr 2010 von FMA und OeNB veröffentlichten „Guiding Principles“ zur Vergabe von Fremdwährungskrediten in CESEE-Ländern, verpflichten sich die österreichischen Bankengruppen, auf die riskantesten Formen von Fremdwährungskrediten zu verzichten. Daran halten sich die österreichischen Banken, wie Analysen der OeNB bestätigen. $\mathrm{Zu}$ den besonders riskanten Fremdwährungskreditformen zählen Konsum- und Hypothekarkredite in Schweizer Franken oder Japanischen Yen an private Haushalte bzw. kleine und mittlere Unternehmen ohne währungskongruente Einkommen sowie von Konsumkrediten in Euro an private Haushalte, die nicht über höchste Bonität verfügen. Alle Maßnahmen und Leitlinien der österreichischen Aufsicht sind auf die Stärkung der Finanzmarktstabilität ausgerichtet, die Wachstumsperspektiven sowie der Zugang zu finanziellen Ressourcen sollen dabei so gering wie möglich beeinflusst werden.

\footnotetext{
${ }^{10}$ Die stabilen Rahmenbedingungen umfassen Einlagen von Nichtbanken, von den Tochterbanken außerhalb des eigenen Konzerns begebene Anleihen mit einer (urspr.) Laufzeit von über einem Jahr, supranationales Funding und Kapital von Dritten.
} 
III.12 Im Februar 2012 erstmals vorgelegten „Frühwarnbericht“ der EK (AMR) zu den makroökonomischen Ungleichgewichten wurde zu Österreich u.a. die Überschreitung des Schwellenwertes der Privatsektorverschuldung thematisiert. Die Verschuldung (Kredite und Wertpapiere ohne Anteilsrechte) des Privatsektors belief sich in Österreich 2010 auf rund 474 Mrd. Euro (165,7\% des BIP). Dabei sind seit 2001 vor allem Wohnbaukredite (+92\%) bzw. bei nichtfinanziellen Unternehmen seit 2000 die langfristigen verzinslichen Wertpapiere $(+175 \%)$ angestiegen. Der Anstieg der Verschuldung des Privatsektors hat sich jedoch im Laufe des Jahres 2011 im Vergleich zu den Vorjahren stark eingebremst. Den Verpflichtungen stehen zudem auf der Aktivseite entsprechend hohe Vermögen gegenüber. $\mathrm{Da}$ in Österreich keine Preisblasen mit anschließenden Wertabstürzen evident bzw. zu erwarten sind, gefährdet die Verschuldung des Privatsektors die finanzielle Stabilität nicht. Insgesamt belief sich das gesamte Geldvermögen des Privatsektors in Österreich zuletzt (2010) auf 941 Mrd. Euro, demgegenüber standen Gesamtverpflichtungen in der Höhe von 827 Mrd. Euro. Im Gesamtbild weist der Privatsektor in Österreich damit eine positive Bilanz aus.

III.13 Unter Vermeidung überschießender sowie nicht nachhaltiger Entwicklung ist die Aufnahme und Verfügbarkeit von Fremdkapital ein wesentlicher Faktor für den unternehmerischen Erfolg. Die Kreditkanäle zur Finanzierung der Realwirtschaft funktionieren diesbezüglich in Österreich weiterhin ungestört, die Neukreditvergaben an Unternehmen lagen zuletzt (Q3/2011) mit 17,5 Mrd. Euro auf stabilem Niveau.

\section{Wettbewerbsfähigkeit und Auslandsverschuldung}

III.14 Die Orientierung am EU-Binnenmarkt und die bestmögliche Nutzung internationaler Absatzmöglichkeiten sind für Österreich als kleine, offene Volkswirtschaft wirtschaftspolitisch unverzichtbar. In diesem Sinn weisen die Ergebnisse der letzten Jahre deutlich auf eine zunehmende Wettbewerbsfähigkeit hin: Seit 2002 wurden Leistungsbilanzüberschüsse von durchschnittlich 2,7\% des BIP erzielt. 2011 verringerte sich der Überschuss aufgrund der geringeren Auslandsnachfrage etwas auf 1,7\% des BIP. Mit $+1,4 \%$ (2012) und $+1,8 \%$ (2013) erwartet das WIFO in nächster Zeit aber eine anhaltend stabile Entwicklung.

III.15 Ein Blick auf die Zusammensetzung der Leistungsbilanz in den ersten drei Quartalen 2011 zeigt, dass ein Überschuss von 4,5 Mrd. Euro (2,0\% des BIP Q1-Q3 2011) in erster Linie auf einen positiven Saldo des Reiseverkehrs von 5,4 Mrd. Euro zurückzuführen ist. Deutliche Impulse kamen mit einem Überschuss von 4,8 Mrd. Euro auch aus der Kategorie der unternehmensbezogenen Dienstleistungen. In der Kapitalbilanz nahmen die Direktinvestitionsflüsse in Österreich von 6 Mrd. Euro (Q1-Q3 2010) auf 9,3 Mrd. Euro (Q1Q3 2011) zu, das Engagement im Ausland stieg von 6,2 Mrd. Euro (Q1-Q3 2010) auf 14,8 Mrd. Euro (Q1-Q3 2011).

III.16 Die gemäßigte Entwicklung der nominellen Lohnstückkosten der letzten Jahre wird sich fortsetzen und damit die preisliche Wettbewerbsfähigkeit Österreichs weiterhin stützen. Für 2012 und 2013 werden Anstiege von 3,6\% und 1,3\% erwartet. Auch ein Blick auf den realen effektiven Wechselkurs lässt auf eine relativ hohe Wettbewerbsfähigkeit der österreichischen Wirtschaft schließen. Nach einer geringfügigen Aufwertung $2009(+0,4 \%)$ war für 2010 eine deutliche Abwertung von 2,7\% und für 2011 wieder eine leichte Aufwertung $(+0,7 \%)$ zu verzeichnen.

III.17 Der Außenhandel entwickelte sich in Österreich 2011 dynamisch, die Wareneinfuhren legten im Jahresvergleich um 15,1\% und die Ausfuhren um 11,7\% zu. Der Exportmarktanteil wird im dem AMR zugrundeliegenden Scoreboard für das jeweilige Jahr mit jenem vor fünf Jahren verglichen, weshalb sich die negativen Kriseneffekte im entsprechenden Indikator derzeit vollständig niederschlagen. In Österreich kam es im Krisenjahr 2009 zu einem Einbruch der Exporte von über 20\%. Bereits 2010 konnten die Ausfuhren wieder um 16,7\% 
gesteigert werden, neben dem Zuwachs 2011 ist laut WIFO auch für 2012 trotz Eintrübung der globalen Nachfrage mit einem weiteren beständigen Wachstum zu rechnen.

III.18 Die internationale Vermögensposition hat sich in Österreich in den letzten Jahren grundsätzlich verbessert (2005 - 2010: +12,7 PP), getrieben wurde diese positive Entwicklung in erster Linie durch einen stetigen Aufbau an Aktiva. Im Jahr 2010 lag der Wert bei $-9,8 \%$ des BIP, d.h. deutlich unter der im AMR ausgewiesenen Referenzgröße. Das Fremdwährungsrisiko der öffentlichen Verschuldung ist sehr gering, die Gesamtverschuldung ist zu 99,5\% in Euro denominiert. Insgesamt wird beim Schuldenmanagement auf eine risiko-averse und stabilitätsorientierte Strategie gesetzt, die Aufnahme finanzieller Mittel erfolgt beinahe ausschließlich zu fixer Verzinsung, und das „debt rollover" ist mit zuletzt (2011) 4,9\% des BIP vergleichsweise gering.

\section{Thematische Koordination}

IV.1 Dieser Abschnitt informiert über die Fortschritte hinsichtlich der Erreichung der nationalen Europa 2020 Ziele, die Umsetzung der länderspezifischen Empfehlungen sowie über die Umsetzung der Prioritäten des Jahreswachstumsberichts 2012.

\begin{tabular}{|c|c|c|c|}
\hline & EU-Gesamtziel & $\begin{array}{l}\text { Nation } \\
\text { Öste }\end{array}$ & $\begin{array}{l}\text { les Ziel } \\
\text { reich }\end{array}$ \\
\hline Beschäftigungsquote in \% & $75 \%$ & $\begin{array}{l}2020 \\
77-78 \% \\
\end{array}$ & \begin{tabular}{|l|} 
Stand 2010 \\
$74,9 \%$
\end{tabular} \\
\hline F\&E-Investitionen in \% des BIP & $3 \%$ & $3,76 \%$ & $2,76 \%$ \\
\hline $\begin{array}{l}\text { Emissionsreduktionsziele in den } \\
\text { Nicht-Emissionshandelssektor } \\
\text { (verglichen mit 2005) }\end{array}$ & $\begin{array}{l}-10 \% \text { (gegenüber 2005, } \\
\text { Non ETS) }\end{array}$ & $\begin{array}{l}-16 \% \\
\text { (Basisjahr 2005) }\end{array}$ & $-9,1 \%$ \\
\hline Erneuerbare Energien & $20 \%$ & $34 \%$ & $30,8 \%^{(2)}$ \\
\hline $\begin{array}{l}\text { Energieeffizienz - Reduktion des } \\
\text { Energieverbrauchs in Mio.t ROE }\end{array}$ & $\begin{array}{l}\text { Steigerung der } \\
\text { Energieeffizienz um } \\
20 \% \text { (= 368 Mio.t ROE) }\end{array}$ & 7,16 Mio. t ROE ${ }^{(3)}$ & n.v..$^{(4)}$ \\
\hline Schulabbrecherquote in \% & $10 \%$ & $9,5 \%$ & $8,3 \%$ \\
\hline Anteil der Hochschulabsolventen in \% & $40 \%$ & $\begin{array}{l}38 \% \text { (einschl. } \\
\text { ISCED } 4 a \text {, dessen } \\
\text { Anteil derzeit bei } \\
12 \% \text { liegt) }\end{array}$ & $35,2 \%$ \\
\hline $\begin{array}{l}\text { Senkung der Zahl der von Armut / } \\
\text { sozialer Ausgrenzung bedrohten } \\
\text { Bevölkerung (in Personen) }\end{array}$ & 20000000 & 235.000 & 159.000 \\
\hline
\end{tabular}

(1) Stand 2010 (BMLFUW, Höchstmengen von Treibhausgasemissionen für Österreich im Zeitraum 2013 bis 2020 nach $^{3}$ 4 Abs. 3 Klimaschutzgesetz (KSG) und im Einklang mit der Entscheidung Nr. 406/2009/EG des Europäischen Parlaments und des Rates, 24.2.2012)

(2) Statistik Austria (Energiebilanz 2010)

(3) AEA Berechnung

(4) Nicht verfügbar, da auf EU Ebene noch keine einheitliche Berechnungsbasis vereinbart wurde

\section{IV.1 Beschäftigung}

IV.1.1 Österreich nimmt im internationalen Vergleich eine gute Position im Beschäftigungsbereich ein. Die Beschäftigungsquote der 20- bis 64-Jährigen betrug 2010 74,9\% (5. Rang im EU-Vergleich). Im Vergleich zu 2009 (74,7\%) zeigt sich somit ein leichter 
Anstieg, anhand der Quartalsdaten für 2011 zeichnet sich eine weitere positive Entwicklung ab. ${ }^{11}$

IV.1.2 Die österreichische Bundesregierung hatte bereits in ihrem Nationalen Reformprogramm 2011 die wichtigsten Problemfelder und Herausforderungen für die Arbeitsmarktpolitik identifiziert, die nach wie vor uneingeschränkt Gültigkeit haben. Der Arbeitsmarktbeteiligung älterer ArbeitnehmerInnen und der Steigerung der Erwerbsbeteiligung von Frauen, Jugendlichen und Personen mit Migrationshintergrund kommt höchste Priorität in der österreichischen Arbeitsmarktpolitik zu.

\section{Meilensteinprojekte und neue Initiativen}

- Arbeitsmarktoffensive für ältere und gesundheitlich eingeschränkte Arbeitnehmerlnnen im Rahmen des Stabilitätspakets: 750 Mio. € $2012-2016$

- Einheitliches Pensionskonto (Kontogutschriftspension)

- Frühpension ab dem 62. Lebensjahr (Korridorpension) - Verschärfung der Zugangsvoraussetzungen und Erhöhung des Abschlages

- Schwerpunkt Jugendbeschäftigung

- Pflegefonds: 1,335 Mrd. € $2011-2016$

- Schaffung von Kinderbetreuungsplätzen: 110 Mio. € 2011 - 2014

- Frauenquote für Aufsichtsräte von Unternehmen mit mindestens 50\% staatlicher Beteiligung

- Verpflichtende Einkommensberichte

- Gehaltsrechner

- Lohn- und Sozialdumping-Bekämpfungsgesetz (LSDB-G)

- Kriteriengeleitetes Zuwanderungssystem „Rot-Weiß-Rot Karte“

\section{Arbeitsmarktbeteiligung älterer Arbeitnehmerinnen und Arbeitnehmer}

IV.1.3 In den letzten fünf Jahren konnten bei der Beschäftigungsquote älterer Arbeitnehmerinnen und Arbeitnehmer Steigerungen von mehr als 10 Prozentpunkten erzielt werden.

Beschäftigungsquoten älterer ArbeitnehmerInnen (55-64-jährige) in \% 2010

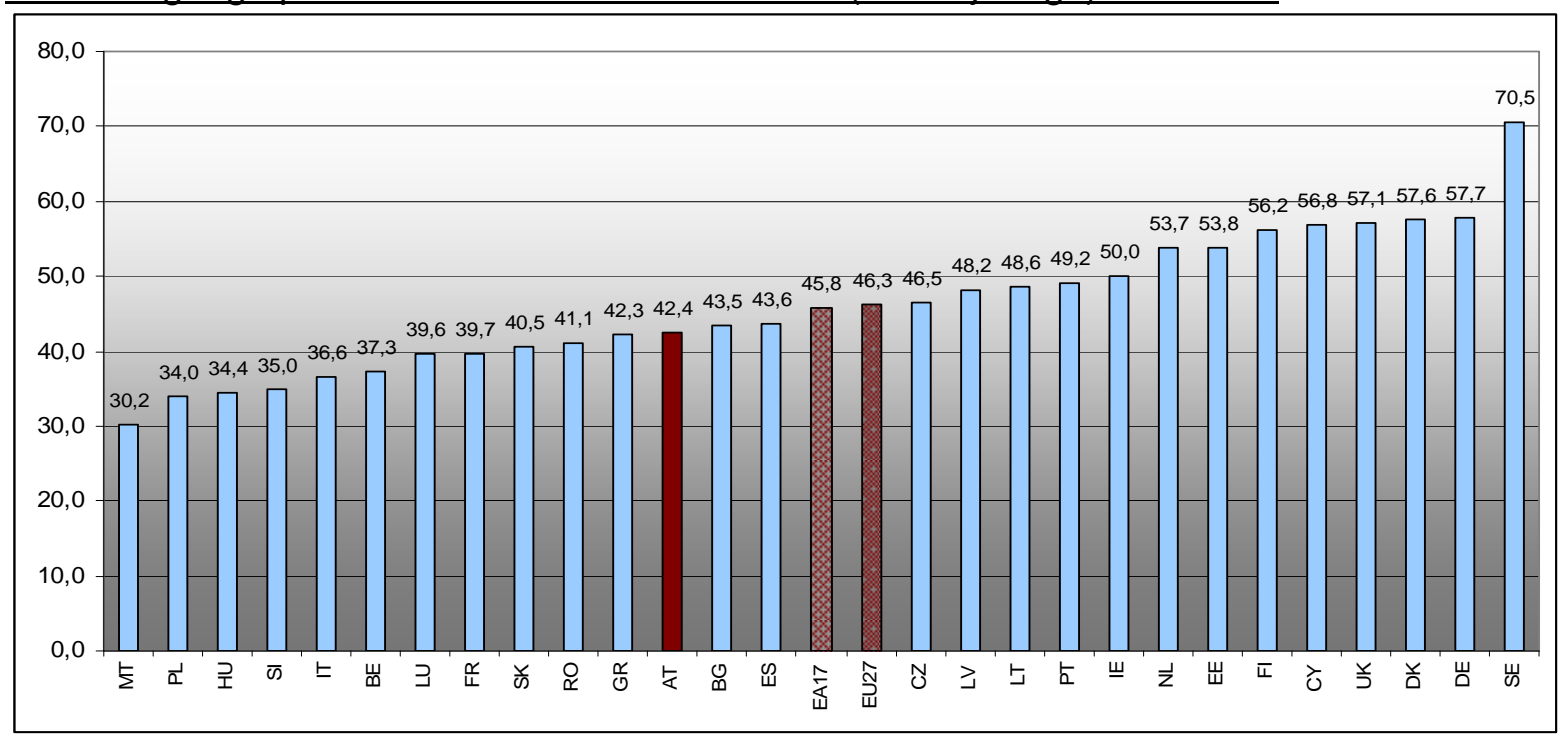

Quelle: Eurostat

\footnotetext{
${ }^{11}$ letztverfügbare Werte von EUROSTAT für Q4/2011 bei 75,3\%
} 
IV.1.4 Das Stabilitätspaket 2012-2016 der Bundesregierung ${ }^{12}$ zielt auch darauf ab, bis zu 195.000 gesundheitlich beeinträchtigte und ältere Menschen länger im Erwerbsleben zu halten. Es setzt auf die Erhöhung des faktischen Pensionsantrittsalters durch

- Fortführung der Reform der Invaliditätspension - Ausweitung von beruflicher Rehabilitation $^{13}$ (siehe auch Armutsziel),

- Verschärfung der Anspruchsvoraussetzungen für die Korridorpension (Pensionsantritt ab dem 62. Lebensjahr mit jährlichen Abschlägen) - diese Pensionsart kann künftig erst nach 40 anstatt von 37,5 Versicherungsjahren in Anspruch genommen werden,

- Erhöhung des Abschlags pro Jahr des vorzeitigen Pensionsantritts (d.h. vor dem Regelpensionsantrittsalter) von $4,2 \%$ auf $5,1 \%$.

IV.1.5 Weitere Anreize zu einem längeren Verbleib im Erwerbsleben soll ab 2014 das so genannte Kontogutschriftmodell setzen. Damit wird ein einheitliches Pensionskonto für alle Versicherten geschaffen. Somit ist die Pensionsberechnung klar nachvollziehbar und der Pensionsanspruch aus der Kontomitteilung ablesbar.

IV.1.6 Im Bereich Arbeitsmarkt ist mit dem Stabilitätsgesetz 2012 beabsichtigt, die Inanspruchnahme des Altersteilzeitgeldes bei kontinuierlicher Arbeitszeitverkürzung nicht nur (wie derzeit) bis zur frühestmöglichen Erfüllung der Anspruchsvoraussetzungen für eine Alterspension, sondern darüber hinaus bis zum gesetzlichen Pensionsalter (max. fünf Jahre) zu ermöglichen. Gleichzeitig mit dieser für Jänner 2013 geplanten Änderung soll die geblockte Variante der Altersteilzeit nur mehr bei Einstellung einer Ersatzkraft (einer vormals arbeitslosen Person oder eines Lehrlings) möglich sein.

IV.1.7 Geplant ist auch, zur Finanzierung von Förderangeboten die Arbeitslosenversicherungs-Beitragspflicht bis 63 Jahre bzw. bis zum Erreichen des Pensionsanspruches auszuweiten. Durch Beschäftigungsförderung für ältere Arbeitslose sollen bis 2016 zusätzlich zu den bestehenden Eingliederungsbeihilfen 40.000 über 50Jährige einen neuen Arbeitsplatz bekommen.

IV.1.8 Rund 70.000 Personen sollen jährlich zusätzlich im Rahmen von „fit2work“ beraten werden, 45.000 ältere und gesundheitlich beeinträchtigte Personen, sollen zusätzlich in AMS-Förderungen einbezogen werden und 32.000 stärker beeinträchtigte, aber noch arbeitsfähige Personen sollen von beruflichen Rehabilitationsmaßnahmen profitieren. In Summe ist geplant, für die Arbeitsmarktoffensive über $€ 750$ Mio. zur Verfügung zu stellen.

\footnotetext{
${ }^{12}$ siehe auch Österreichisches Stabilitätsprogramm. Fortschreibung für die Jahre 2011 bis 2016, Bundesministerium für Finanzen, April 2012

${ }^{13}$ Für Menschen unter 50 Jahren, die unter Berücksichtigung ihrer gesundheitlichen Entwicklung eine Chance auf Wiedereingliederung in den Arbeitsmarkt haben, sollen Invaliditätspensionen durch Leistungen des Arbeitsmarktservice ersetzt und eine Wiedereingliederung in den Arbeitsmarkt angestrebt werden. Sind die Voraussetzungen für eine Pension aus dem Versicherungsfall der geminderten Arbeitsfähigkeit zumindest „wahrscheinlich“ erfüllt, so besteht ein Rechtsanspruch auf berufliche Rehabilitation. Diese Neuregelungen werden im Laufe des Jahres 2012 verhandelt und abgeschlossen.
} 


\section{Strategische Ausrichtung und Umsetzung bestehender Maßnahmen}

IV.1.9 Der Erhöhung des effektiven Pensionsantrittsalters kommt oberste Priorität zu. Die im NRP 2011 angeführten Maßnahmen betreffend Invaliditätspension und besondere Pensionsbestimmungen für Langzeitversicherte (Anpassung der geltenden Regelung) werden konsequent umgesetzt. Um Frühpensionierungen Schritt für Schritt zurückzudrängen, liegt der Schwerpunkt der Maßnahmen hier auf der besseren Prävention während der aktiven Zeit.

IV.1.10 Im Rahmen des Programms „fit2work ${ }^{14}$ " findet

Länderspezifische Empfehlung Nr. 3: „... Zugang zur derzeitigen Frühpensionierungsregelung für Langzeitversicherte begrenzen ...." unter anderem Beratung zur Prävention sekundärer Berufskrankheiten statt. Anhand dieses Programms soll bis 2013 der Antritt des vorzeitigen Ruhestands aufgrund von Krankheiten massiv hinausgezögert werden.

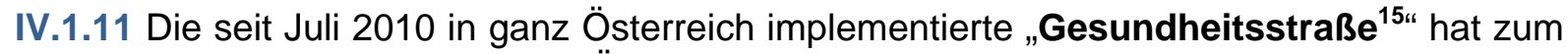
Ziel, die Beschäftigungsquote der Älteren anzuheben und die Zahl der Invaliditätspensionen abzubauen. Das Projekt wird begleitend evaluiert.

IV.1.12 "Check 4 Chances“ ist ein von September 2011 bis August 2012 laufendes Pilotprojekt, das Arbeitsfähigen nach Durchlaufen der Gesundheitsstraße konkrete Schritte für eine berufliche Integration aufzeigt. Nach erfolgter Bewertung soll ein weiterer Ausbau überlegt werden.

IV.1.13 Um ältere Personen länger in Beschäftigung zu halten bzw. ihre Arbeits- und Beschäftigungsfähigkeit zu erhalten und zu verbessern, wurde im Jahr 2011 im Budgetbegleitgesetz der Paradigmenwechsel „Rehabilitation vor Pension“ verankert.

IV.1.14 Mit dem Forschungsprojekt Prototyp „Wanderkarte“ für gesunde Berufswege wird dem vorzeitigen Ausscheiden aus dem Berufsleben durch das Aufzeigen beruflicher Alternativen für Personen in Berufen mit begrenzter Tätigkeitsdauer (bei anhaltend hoher physischer und psychischer Belastung) entgegengewirkt.

IV.1.15 Durch eine alter(n)sgerechte Arbeitsgestaltung und Präventionsmaßnahmen nach dem Arbeitnehmerlnnenschutzgesetz (ASchG) werden die Arbeitsbedingungen für ältere Arbeitnehmerlnnen verbessert - zur Förderung und zum Erhalt der Arbeitsfähigkeit der Beschäftigten und im Sinne eines menschengerechten Generationenmanagements in der Arbeitswelt. Die Präventionsziele der österreichischen Arbeitsschutzstrategie 2007-2012 (Folgestrategie bis 2020) sollen unter Berücksichtigung von Gender- und Altersaspekten erreicht werden. Die Arbeitsinspektion führt dazu seit 2007 Beratungs- und Kontrollschwerpunktaktionen in Klein- und Mittelbetrieben durch, um die Unternehmen zu motivieren eine „Altersstrukturanalyse" durchzuführen, alterskritische Arbeitsvorgänge und Einwirkungen $\mathrm{zu}$ ermitteln und alter(n)sförderliche Arbeitsbedingungen zu schaffen. Der Arbeitsinspektionsfolder „Alter(n)sgerechte Arbeitsgestaltung - Mit Arbeit gesund älter werden" informiert über die Umsetzung der Gefährdungsbeurteilung zum Sicherheits- und Gesundheitsschutz für ältere Beschäftigte. Für die Durchführung einer Altersstrukturanalyse im Rahmen der Evaluierung hat die Arbeitsinspektion ein Erhebungstool entwickelt, das den

\footnotetext{
${ }^{14}$ Siehe auch Maßnahmenübersicht zum Nationalen Reformprogramm 2011

${ }^{15}$ Siehe auch Maßnahmenübersicht zum Nationalen Reformprogramm 2011
} 
Betrieben zur Verfügung gestellt wird und wie der Folder auf der Arbeitsinspektionswebsite ${ }^{16}$ verfügbar ist. Im Rahmen der Qualifizierungsberatung für Betriebe und Flexibilitätsberatung erhalten Unternehmen kostenlos Beratungsleistung zur Unterstützung der Personalentwicklung, Bildungsplanung und Umstrukturierung. Seit 2011 wird ein Schwerpunkt auf alternsgerechte Gestaltung der Arbeitsplätze gelegt.

\section{Steigerung der Erwerbsbeteiligung bestimmter Zielgruppen}

IV.1.16 Die Anhebung der Erwerbsbeteiligung von bestimmten Zielgruppen, insbesondere von Frauen, Jugendlichen und Personen mit Migrationshintergrund stellt nach wie vor eine zentrale beschäftigungspolitische Herausforderung für die österreichische Arbeitsmarktpolitik dar.

IV.1.17 Im Rahmen des Euro-Plus-Pakts hat Österreich einen Schwerpunkt auf die Jugendbeschäftigung gelegt.

\section{Nationaler Beschäftigungsplan - „National Job Plan“ und Maßnahmen im Rahmen des Euro-Plus-Paktes}

\section{Jugendliche}

Österreich unterstützt die verstärkten Anstrengungen zur Bekämpfung der Jugendarbeitslosigkeit auf EU-Ebene im Sinne einer europäischen Jugendinitiative, wie sie in der Mitteilung der Europäischen Kommission "Chancen für junge Menschen“, in der Erklärung der Staats- und Regierungschefs vom 30. Jänner 2012, im Gemeinsamen Beschäftigungsbericht und im Synthesebericht zur Umsetzung des Europäischen Semesters dargestellt werden ${ }^{17}$, und hat bereits mit den im Anhang II des Nationalen Reformprogramms 2011 genannten Euro-Plus-Pakt-Maßnahmen zur Beschäftigungsförderung einen deutlichen Schwerpunkt auf junge Menschen gesetzt.

Im nationalen Kontext wird der Arbeitsmarkt- und Beschäftigungspolitik für Jugendliche im Jahr 2012 erneut höchste Priorität eingeräumt. Im Jahr 2011 standen für die Förderung von Lehrverhältnissen, Beschäftigung und Qualifizierung Jugendlicher mit mehr als 600 Mio. $€$ aus Mitteln des Arbeitsmarktservice, des Insolvenz-Entgelt-Fonds und des Bundessozialamtes zur Verfügung - damit sind Jugendliche die am intensivsten unterstützte Zielgruppe der österreichischen Arbeitsmarktpolitik. Der Fördermitteleinsatz bleibt auch 2012 auf stabilem Niveau.

Mit der Umsetzung der Maßnahme Jugendcoaching (siehe auch Bildungs- und Armutsziel) wird ab 2012 vorerst in den Bundesländern Wien und Steiermark ein Beratungs- und Betreuungsangebot zur Begleitung von ausgrenzungsgefährdeten SchülerInnen im 9 . Schulbesuchsjahr etabliert. Der Vollausbau in allen Bundesländern ist stufenweise bis zum Jahr 2014 geplant. Ziel des Jugendcoaching ist es vor allem,

- ausgrenzungs-und schulabbruchgefährdete SchülerInnen zu beraten und zu begleiten, sodass der individuell richtige Bildungsweg gefunden wird,

- verstärkt Orientierung in Bezug auf die Bildungs- und Berufslaufbahn zu geben, auch für Jugendliche mit sonderpädagogischem Förderbedarf (die bis 2011 durch die Maßnahme Clearing betreut wurden),

- psychosoziale Probleme aufzufangen, die den Bildungsweg gefährden könnten,

\footnotetext{
${ }^{16}$ Siehe http://www.arbeitsinspektion.gv.at/Al/Gesundheit/Arbeitswelt/default.htm

${ }^{17}$ Insbesondere die Verweise auf eine „Jugendgarantie“, mit der "gewährleistet werden soll, dass alle jungen Menschen innerhalb von vier Monaten nach Verlassen der Schule eine Arbeitsstelle oder einen Ausbildungs- oder Weiterbildungsplatz erhalten“ (KOM(2011) 933 endg, S. 11)
} 
- Weitervermittlung zu anderen Beratungsstellen bzw. auch zu Praktikumsplätzen.

Beim Erfolgsfaktor duale Berufsausbildung wurden in der betrieblichen Lehrausbildung neue Akzente zur Absicherung des individuellen Ausbildungserfolgs gesetzt. Das mit der Novelle zum Berufsausbildungsgesetz 2011 verankerte Lehrlingscoaching ermöglicht ab 2012 den Aufbau eines Beratungs-, Begleitungs- und Unterstützungsangebots für Lehrlinge und Unternehmen u.a. zur Vermeidung von Lehrabbrüchen. Dabei sollen basierend auf den positiven Erfahrungen mit der Berufsausbildungsassistenz Informationen bereitgestellt, regelmäßig Kontakt $\mathrm{zu}$ Lehrling, Betrieb und Berufsschule gehalten und die Qualitätssicherung gefördert werden.

Die bereits im Nationalen Reformprogramm 2011 beschriebenen Maßnahmen „Aktion Zukunft Jugend" und Ausbildungsgarantie ${ }^{18}$ werden weitergeführt. Die Umsetzung der Überbetrieblichen Lehrausbildung (ÜBA) erfolgt auch im Ausbildungsjahr 2011/2012 auf hohem Niveau. Damit wird jenen Jugendlichen, die keine betriebliche Lehrausbildung absolvieren können, die Chance auf eine hochwertige Fachausbildung eröffnet. Eine Evaluierung der arbeitsmarktpolitischen Effekte der überbetrieblichen Lehrausbildung bestätigt, dass die ÜBA einen nachhaltigen Beitrag zur Verbesserung der Arbeitsmarktsituation jener Jugendlichen leistet, die durch sie einen Lehrabschluss erreichen.

Die Unterstützungsinstrumente für die Lehrlingsausbildung in den Betrieben sollen mit folgenden Maßnahmen weiterentwickelt werden:

- Gestaltung der betrieblichen Lehrstellenförderung entsprechend den Ausbildungserfordernissen der Unternehmen (laufender Prozess)

- Einheitliche Standards bei der Lehrabschlussprüfung durch Überprüfung von Prüfungsbeispielen durch „Clearingstelle“ (ab 2012)

- Praxisrelevante Ausbildungsmaterialien für Ausbilder, ergänzend zu den Berufsbildern (ab 2012)

Folgende Maßnahmen werden in der außerschulischen Jugendförderung fortgeführt:

- Bundes-Jugendförderung: Schwerpunktförderung Berufsorientierung

- Jobtalks 2.0

Die Jugend-Implacementstiftung „Just NEU“ sowie die zwanzig Produktionsschulen ${ }^{19}$ werden auch im Jahr 2012 fortgeführt.

Im Sinne einer zukunftsorientierten Integrationspolitik sieht der Nationale Aktionsplan für Integration die Forcierung der Arbeitsmarktintegration von Jugendlichen mit Migrationshintergrund vor. Junge MigrantInnen, insbesondere bildungsferne Jugendliche, sollen verstärkt in Qualifizierungsmaßnahmen für Berufe einbezogen werden, für die am Arbeitsmarkt eine starke Nachfrage besteht. Eine gerade im Hinblick auf die Zielgruppe der jugendlichen MigrantInnen wichtige Rolle spielen die zur Unterstützung des Berufseinstiegs auf- und ausgebauten Programme wie die Förderung der überbetrieblichen Lehrausbildung oder niederschwelligere Angebote in diesem Bereich wie die „Produktionsschulen“. Profitieren sollen Jugendliche mit Migrationshintergrund darüber hinaus aber auch von den zur Dropout-Vermeidung eingesetzten Maßnahmenprogrammen des Jugend- und Lehrlingscoachings.

\footnotetext{
18 Siehe auch Maßnahmenübersicht Nationales Reformprogramm 2011

${ }^{19}$ Siehe auch Maßnahmenübersicht Nationales Reformprogramm 2011
} 


\section{Pensionen}

Im Zusammenhang mit der Sicherstellung der langfristigen Finanzierbarkeit des öffentlichen Pensionssystems wird eine Reihe von Maßnahmen zur Erhöhung des effektiven Pensionsantrittsalters durchgeführt.

- Weiterführung der Reform des Invaliditätspensionsrechtes: Ausbau der gesundheitlichen und beruflichen Rehabilitation;

- Stufenweise Anhebung des für den Tätigkeitsschutz (Schutzbestimmung für die Inanspruchnahme einer Invaliditätspension) maßgeblichen Alters vom 57. auf das 60 . Lebensjahr.

\section{Frauen}

IV.1.18 Die Beschäftigungsquote der 20- bis 64-jährigen Frauen lag im Jahr 2010 mit 69,6\% deutlich über dem EU-Schnitt von 62,1\%. Gleichzeitig war die Teilzeitquote mit 43,8\% ebenfalls weit überdurchschnittlich. ${ }^{20}$ Ziel der Arbeitsmarktpolitik ist, die Erwerbsbeteiligung von Frauen durch gezielte Maßnahmen und Angebote zu erhöhen und Frauen besonders nach Phasen der Kinderbetreuung beim Wiedereinstieg zu unterstützen. Generell werden Frauen überproportional zu ihrem Anteil an den registrierten Arbeitslosen gefördert, indem innen 50\% des aktiven Förderbudgets zukommen. 2011 wurden rund 193.600 Frauen gefördert, der Großteil (82\%) nahm an Qualifizierungen teil. Insgesamt wurde für diese Zielgruppe knapp 454 Mio. € ausgegeben.

\section{Neue Initiativen und geplante Maßnahmen}

IV.1.19 Der 2011 eingerichtete Pflegefonds (siehe auch Armutsziel) unterstützt den bedarfsgerechten Auf- und Ausbau sozialer Dienstleistungen für ältere und pflegebedürftige Personen. Durch die

Länderspezifische Empfehlung Nr. 4: „,... hohe Arbeitsplatzintensität hat er eine Verbesserungen in Bezug auf das Angebot an (...) starke Beschäftigungswirkung von 29.000 Pflegediensten vorzunehmen ...." Pflege-Arbeitsplätzen und entfaltet somit auch starke volkswirtschaftliche und konjunkturelle Effekte. Mit dem Pflegefondsgesetz wird u.a. die Weiterentwicklung der bedarfsgerechten Versorgung pflegebedürftiger Personen und ihrer Angehörigen mit bedürfnisorientierten und leistbaren Betreuungs- und Pflegedienstleistungen im Bereich der Langzeitpflege unterstützt.

\section{Strategische Ausrichtung und Umsetzung bestehender Maßnahmen}

IV.1.20 2011 wurden zusätzlich Mittel für das Programm „Frauen in Technik““21 aufgewendet, um Frauen verstärkt im handwerklich-technischen Bereich zu qualifizieren und dadurch ihre Einkommens- und Arbeitsmarktchancen nachhaltig zu verbessern. Das FiTProgramm wurde bis 2014 verlängert. Aktuell werden Frauenberufszentren ${ }^{22}$, welche die Programmschiene „Laufbahnberatung für Frauen“ anbieten, in drei Bundesländern pilotiert. Über einen flächendeckenden Ausbau wird nach der Evaluierung im Jahr 2012 entschieden.

IV.1.21 Mit dem bereits österreichweit angebotenen Programm „Wiedereinstieg mit Zukunft" werden Frauen nach einer Phase der Kinderbetreuung beim Wiedereinstieg unterstütz. Für 2012 ist geplant, dass rund 4.000 Frauen dieses Angebot nach der Babypause in Anspruch nehmen können.

\footnotetext{
${ }^{20}$ cf. Eurostat

${ }^{21}$ Siehe auch Maßnahmenübersicht des Nationalen Reformprogramms 2011

22 Siehe auch Maßnahmenübersicht des Nationalen Reformprogramms 2011
} 
IV.1.22 Das Projekt „Kompetenz mit System“ ermöglicht Personen, die keine durchgehende Ausbildung absolvieren können oder die häufig von Arbeitslosigkeit betroffen sind, Qualifizierungen für bestimmte Berufe bis hin zum Lehrabschluss in modularer Form zu absolvieren. Dadurch sollen fachliche Schulungen des Arbeitsmarktservice stärker in den Kontext formaler Ausbildungsabschlüsse gestellt werden. Nach Pilotierung in mehreren Bundesländern ist für 2012 die flächendeckende Einführung geplant. ${ }^{23}$.

IV.1.23 Die Verringerung der Segmentation am Arbeitsmarkt sowie des Verdienstunterschieds zwischen Frauen und Männern, die Schaffung der Rahmenbedingungen für die Vereinbarkeit von Beruf und Betreuungspflichten und die Beseitigung der „gläsernen Decke“, die Frauen von führenden Positionen

Länderspezifische Empfehlung Nr. 4: „,... den nationalen Aktionsplan zur Gleichbehandlung von Frauen und Männern auf dem Arbeitsmarkt umsetzen, (...) und den hohen geschlechtsspezifischen Lohnunterschied reduzieren ..." ausschließt, hängen eng miteinander zusammen und sind strategische Schwerpunkte. Dabei liegt ein besonderer Fokus auf qualitativ hochwertigen und existenzsichernden Arbeitsplätzen. Dies ist auch unter dem Aspekt der Armutsgefährdung zentral.

IV.1.24 Das Bundesministerium für Frauen und Öffentlicher Dienst hat den Nationalen Aktionsplan zur Gleichstellung von Frauen und Männern am Arbeitsmarkt im Jahr 2010 vorgelegt. Die Umsetzung der darin enthaltenen Maßnahmen wurde weiter vorangetrieben, $45 \%$ der Maßnahmen sind bereits umgesetzt. Die erste Analyse zur Umsetzung wurde 2011 publiziert, der nächste Bericht ist 2013 geplant.

IV.1.25 Die Bundesregierung hat sich 2011 zu einer Frauenquote für die Aufsichtsräte staatsnaher Unternehmen (Unternehmungen an denen der Bund mit 50\% und mehr beteiligt ist) verpflichtet. Bis Ende 2013 sollen 25\% und bis Ende $2018 \quad 35 \%$ der Aufsichtsratsmitglieder, die der Bund entsendet, Frauen sein. Es wird angestrebt, diese Quoten auch im gesamten Gremium zu erreichen. Aktuell wird die jährliche Fortschrittserhebung durchgeführt. Die anzustrebende Frauenquote im Bundesdienst wurde mit 1. Jänner 2012 auf 50\% erhöht. Aktuell ist ein Leitfaden zur betrieblichen Frauenförderung in Vorbereitung. Darüber hinaus werden das Führungskräfteprogramm Zukunft.Frauen und die Aufsichtsrätinnen-Datenbank, um hochqualifizierte Kandidatinnen für Aufsichtsratsfunktionen sichtbarer zu machen, fortgesetzt.

IV.1.26 Um den geschlechtsspezifischen Verdienstunterschied zu verringern, wird strategisch auf Transparenz der Einkommen gesetzt. Per Gesetz wurden 2011 verpflichtende Einkommensberichte für Unternehmen eingeführt, beginnend mit Unternehmen mit mehr als 1.000 Beschäftigten. Seit 1. Jänner 2012 sind auch Einkommensberichte ab über 500 MitarbeiterInnen verpflichtend, und bis 2014 gilt dies für alle Betriebe mit mehr als 150 MitarbeiterInnen. Ein, gemeinsam mit den Sozialpartnern erarbeiteter Leitfaden für die Erstellung des Einkommensberichts, wie auch Workshops zu diesem Thema, die vom Bundesministerium für Frauen angeboten werden, unterstützen die Unternehmen bei Erstellung des Einkommensberichts.

IV.1.27 In Stelleninseraten muss das zu erwartende Gehalt (zumindest der kollektivvertragliche Mindestlohn und gegebenenfalls die Möglichkeit der Überzahlung)

\footnotetext{
${ }^{23}$ Das oberösterreichische Projekt „Du kannst was!“ mit ähnlicher Zielsetzung ermöglicht Personen, die keine (österreichische) Berufsausbildung haben, aber im Laufe ihres Arbeitslebens praktische Erfahrungen gesammelt haben, einen anerkannten Abschluss in einem von neun Lehrberufen.
} 
angegeben werden, Verstöße werden seit 1. Jänner 2012 sanktioniert. Seit Inkrafttreten der Sanktionen halten $85 \%$ der Jobinserate diese Verpflichtung gesetzeskonform ein. ${ }^{24}$ Seit 3. Oktober 2011 steht das Online-Tool "Gehaltsrechner" (www.gehaltsrechner.at) zur Verfügung. Der Gehaltsrechner gibt den Durchschnittsverdienst für ein konkretes Profil (Alter, Ausbildung, Berufserfahrung, Tätigkeit, Branche) an. Er unterstützt damit die Orientierung bei Berufseinstieg und Gehaltsverhandlungen. Die Anwaltschaft für Gleichbehandlung und die Senate der Gleichbehandlungskommission können seit 2011 im Einzelfall bei vermuteter Entgeltdiskriminierung Einkommensdaten von Vergleichspersonen bei der Sozialversicherung einholen.

IV.1.28 Um Frauen eine Vollzeitbeschäftigung leichter ermöglichen zu können, ist es

Länderspezifische Empfehlung Nr. 4: „,... notwendig, die Rahmenbedingungen zu verbessern. Dies erfolgt einerseits über den Verbesserungen in Bezug auf das Angebot an Ganztagesbetreuungseinrichtungen vornehmen ...." Ausbau des institutionellen Kinderbetreuungsangebots, ${ }^{25}$ andererseits durch strukturelle Verbesserungen im Pflegeund Versorgungsbereich für ältere Menschen.

IV.1.29 Um in allen Regionen ein bedarfsgerechtes Angebot für Eltern und ihre

Kinder bieten zu können, stellte der Bund den Ländern und Gemeinden 2011 Zweckzuschüsse von 10 Mio. € und 2012 - 2014 in der Höhe von insgesamt 45 Mio. $€$ zur Verfügung. Die Bundesländer stellen für diesen Zweck im Zeitraum 2011 bis 2014 ebenfalls 55 Mio. $€$ zur Verfügung. Damit werden der Ausbau des institutionellen Betreuungsangebots für Kinder bis zum Schuleintritt sowie die Forcierung des Tageselternangebots unterstützt. Dabei liegt der Schwerpunkt auf der Schaffung von Betreuungsplätzen für unter-dreijährige Kinder. Damit wird das Erreichen der Betreuungsquote gemäß dem Barcelona-Ziel der EU (33\%) auch in dieser Altersgruppe weiter vorangetrieben. Da bei den 3- bis 6-Jährigen das Barcelona-Ziel mit 93,4\% bereits erreicht wurde, sollen hier künftig nur noch Betreuungsplätze zur Schließung von regionalen Betreuungslücken gefördert werden.

IV.1.30 Mit einer Offensivmaßnahme soll die Ganztagesbetreuung an Schulen bis 2016 deutlich ausgebaut werden. Die Angebote umfassen Ganztagsschulen, qualifizierte Lernbetreuung, Nachmittagsbetreuung, Mittagessen und Freizeitaktivitäten (siehe Bildungskapitel).

\footnotetext{
${ }^{24}$ Analyse der Arbeiterkammer, Februar 2012

${ }^{25}$ Siehe auch Maßnahmenübersicht Nationales Reformprogramm 2011
} 


\section{Personen mit Migrationshintergrund}

IV.1.32 Die Arbeitsmarktsituation von MigrantInnen ist von häufigen Wechseln zwischen Arbeitslosigkeit und Beschäftigung gekennzeichnet. Daher setzt die Arbeitsmarktpolitik in

Länderspezifische Empfehlung Nr. 4: „,... die Erwerbsbeteiligung steigern ..." den kommenden Jahren auf Maßnahmen, die eine möglichst nachhaltige Beschäftigungsintegration ermöglichen. Um eine optimale und bedarfsorientierte Unterstützung zu gewährleisten, wurden Migranten und

Migrantinnen als eigene Zielgruppe in die Zielvorgaben an das Arbeitsmarktservice (AMS) aufgenommen; dazu wird ab dem Jahr 2012 der Migrationshintergrund von arbeitslos oder arbeitssuchend vorgemerkten Personen ermittelt.

\section{Neue Initiativen und geplante Maßnahmen}

IV.1.33 Österreich hat mit 1. Juli 2011 ein neues kriteriengeleitetes Zuwanderungssystem („Rot-Weiß-Rot-Karte“) ${ }^{26}$ für Arbeitskräfte aus Drittstaaten eingeführt. Hochqualifizierte, Schlüsselarbeitskräfte, Arbeitskräfte in Mangelberufen sowie ausländische AbsolventInnen österreichischer Universitäten erhalten einen auf Dauer ausgerichteten Zugang zum Arbeitsmarkt. Dabei werden Zulassungskriterien wie Qualifikation, Berufserfahrung, Alter und Sprachkenntnisse nach einem Punktesystem geprüft und bewertet. Ziel der neuen Regelung ist es, mit Rücksicht auf die Arbeitsmarkt- und Wirtschaftsentwicklung qualifizierte Schlüsselund Fachkräfte, die am Arbeitsmarkt nicht ausreichend zur Verfügung stehen, für Österreich zu gewinnen, um so den Wirtschaftsstandort abzusichern und das Beschäftigungswachstum zu unterstützen.

IV.1.34 Die österreichische Bundesregierung hat sich zum Ziel gesetzt, die Anerkennung von im Ausland erworbenen Qualifikationen zu erleichtern und zu beschleunigen. In den Bundesländern sollen Anlaufstellen eingerichtet werden, welche InteressentInnen die individuell erforderlichen Informationen bereitstellen und sie bei allen weiteren Schritten begleiten. Diese neuen Stellen sollen in enger Kooperation mit dem AMS tätig werden, um die Arbeitsmarktintegration von MigrantInnen entsprechend ihrer Qualifikation bestmöglich zu unterstützen. ${ }^{27}$

\section{Strategische Ausrichtung und Umsetzung bestehender Maßnahmen}

IV.1.35 Neben der gesamten Förderpalette des AMS werden eigene Förderprogramme speziell für MigrantInnen angeboten. Um eine bedarfsgerechte Betreuung dieser Personengruppe zu gewährleisten werden Dolmetschdienste zugekauft, muttersprachliche Erstinformationsveranstaltungen angeboten sowie fremdsprachige Broschüren und Folder aufgelegt. Da knapp ein Drittel der MigrantInnen als höchste abgeschlossene Ausbildung maximal einen Pflichtschulabschluss aufweist, werden der Erwerb von Basisqualifikationen und Deutschkurse, die u.a. auf die Verbesserung der mündlichen Ausdrucksfähigkeit abzielen, gefördert. Bei Fachkräfteausbildungen werden fachspezifische begleitende Deutschkurse angeboten.

IV.1.36 Im Projekt „Mentoring für MigrantInnen“28 wurden bisher mehr als 500 Mentoringpaare gebildet, rund $40 \%$ der Mentees konnten am österreichischen Arbeitsmarkt Fuß fassen. Aktuell sind weitere Durchgänge am Laufen.

\footnotetext{
${ }^{26}$ Siehe auch Maßnahmenübersicht des Nationalen Reformprogramms 2011

${ }^{27}$ Zusätzlich zu einer neuen Broschüre können Personen, die im Ausland Ausbildungen absolviert und Berufe erlernt haben, ihre für die Anerkennung zuständige Behörde auf der neuen Website www.berufsanerkennung.at ermitteln.

${ }^{28}$ Siehe auch Maßnahmenübersicht des Nationalen Reformprogramms 2011
} 


\section{Reduzierung der Steuer- und Abgabenlast von ArbeitnehmerInnen}

IV.1.37 Mit dem vom Nationalrat beschlossenen Stabilitätspaket 2012-2016 wird die Anhebung von Massensteuern sowie

Länderspezifische Empfehlung Nr. 4: „....die effektive Steuer- und Sozialversicherungsbelastung für Niedrig- und eine zusätzliche Belastung von geringeren und mittleren Durchschnittslohnempfänger, budgetneutral reduzieren ..." Einkommen vermieden. Besserverdienende tragen über einen Solidarbeitrag zur Budgetkonsolidierung bei, für Unternehmerinnen und Unternehmer wird analog dazu der Gewinnfreibetrag eingeschränkt ${ }^{29}$.

\section{Qualität der Arbeit}

IV.1.38 Qualität der Arbeit ist der Schlüssel für mehr und bessere Arbeitsplätze. Gute Arbeit definiert sich über die persönliche Zufriedenheit der Menschen, über leistungsgerechte Entlohnung, aber auch über eine familienfreundliche Gestaltung der Arbeitswelt. Wesentlich für die Qualität der Arbeit ist die Gewährleistung und kontinuierliche Verbesserung der Arbeitsbedingungen (Sicherheit und Gesundheitsschutz am Arbeitsplatz - Prävention von Arbeitsunfällen, arbeitsbedingten Erkrankungen und Berufskrankheiten, Arbeitszeit und Verwendungsschutz, Bewusstseinsbildung).

IV.1.39 Das Lohn- und Sozialdumping-Bekämpfungsgesetz (LSDB-G) ${ }^{30}$ dient der Sicherung gleicher Arbeits- und Lohnbedingungen für alle in Österreich tätigen Arbeitnehmerlnnen. Mit dem LSDG-G wurde in Österreich erstmals eine behördliche Kontrolle des so genannten Grundlohns eingeführt. Die Neuerungen sind mit 1. Mai 2011 in Kraft getreten. Unterschreitungen des (gesetzlich, aufgrund der Verordnung oder Kollektivvertrag) zustehenden Grundlohns sind mit Verwaltungsstrafen bedroht. Damit dieses Niveau gehalten wird, stellt das LSDB-G neben Kontrollen und Verwaltungssanktionen weitere Durchsetzungsinstrumente zur Verfügung. Durch seine Zielrichtung ist das LSDG-B auch geeignet, einen Beitrag zur Armutsbekämpfung zu leisten, da mit diesem Gesetz sichergestellt werden soll, dass ArbeitnehmerInnen ihren Lohn auch tatsächlich erhalten.

IV.1.40 Durch die Bildungskarenz haben ArbeitnehmerInnen die Möglichkeit, sich im Rahmen ihres bestehenden Arbeitsverhältnisses für die Dauer von mindestens zwei Monaten bis zu einem Jahr freistellen zu lassen, um sich weiterzubilden. Die Bildungskarenz dient dem Erwerb von Zusatzqualifikationen und Fertigkeiten und ist ein wichtiges Instrument zur Förderung der Beschäftigungsfähigkeit. Die zuletzt eingeführte, befristete Erleichterung des Zugangs zur Bildungskarenz - nämlich die Herabsetzung des zugrundeliegenden Arbeitsverhältnisses auf sechs Monate - wurde nach erfolgter Evaluierung der Maßnahmenwirkungen seit Beginn des Jahres 2012 unbefristet verlängert.

\section{ESF Programm Beschäftigung}

IV.1.41 Auch das österreichische ESF-Programm „Beschäftigung“ weist eine hohe Übereinstimmung mit den Europa 2020-Politikfeldern auf. Österreich erhält allerdings als wirtschaftsstarker Mitgliedstaat nur einen relativ kleinen Anteil aus den EU-Strukturfonds. Das bedeutet, dass - trotz hoher Kohärenz der österreichischen Strukturfondsprogramme mit den EU-Zielen - nur ein kleiner Teil der Maßnahmen aus EU-Mitteln finanziert werden

\footnotetext{
${ }^{29}$ Details siehe Österreichisches Stabilitätsprogramm für die Jahre 2011 bis 2016

${ }^{30}$ Siehe auch Maßnahmenübersicht Österreichisches Reformprogramm 2011
} 
kann. Der ESF unterstützt in Österreich Maßnahmen für Jugendliche (mit Behinderung), für Personen mit Migrationshintergrund und Niedrigqualifizierte, für Wiedereinsteigerinnen, gehörlose und blinde bzw. hochgradig sehbehinderte Frauen, Frauen mit Kinderbetreuungspflichten sowie für Ältere (mit Behinderung) ${ }^{31}$.

\section{IV.2 Forschung und Entwicklung}

IV.2.1 Die wichtigsten Herausforderungen in diesem Bereich wurden im Österreichischen Reformprogramm 2011 dargelegt. Neben der Unterstützung der Innovationskraft der Unternehmen und der Stärkung der Forschung wurden auch im Euro-Plus-Pakt Schwerpunkte gesetzt, um die Agenda im Rahmen der Europa 2020 Strategie zu intensivieren.

\section{Meilensteinprojekte und neue Initiativen}

- Task-Force Forschung Technologie und Innovation

- Erhöhung des Deckels für Auftragsforschung bei der Forschungsprämie von $100.000 €$ auf 1 Mio. $€$

- Forschungskompetenzen für die Wirtschaft

- Intelligente Produktion

- Institute for Science and Technology Austria (ISTA)

- Vienna Biocenter - Vision 2020

- Young Science - Netzwerk und Service

- Offensivmittel für die Stärkung der Forschung

- Innovationsorientierte öffentliche Beschaffung

IV.2.2 Zentrale Maßnahme für den Zeitraum bis 2020 ist die im März 2011 von der Bundesregierung beschlossene Strategie für Forschung, Technologie und Innovation $(\mathrm{FTI})^{32}$. Österreich setzt sich darin zum Ziel, bis 2020 zu einem der innovativsten Länder der EU zu werden und Forschung, Technologie und Innovation für die gesellschaftlichen und wirtschaftlichen Herausforderungen (z.B. Klimawandel, Ressourcenknappheit, demographischer Wandel) zu mobilisieren. Darüber hinaus leistet Österreich mit der FTIStrategie auch einen wichtigen Beitrag zur Umsetzung der Leitinitiative "Innovation Union“ und trägt dem dort vorgesehenen Selbstbewertungsinstrument weitgehend Rechnung. ${ }^{33}$

IV.2.3 Zur systematischen Umsetzung der FTI-Strategie wurde 2011 unter dem Vorsitz des Bundeskanzleramtes gemeinsam mit den relevanten Bundesministerien auf hoher Verwaltungsebene die Task Force FTI eingerichtet. Darüber hinaus soll der Rat für Forschung und Technologieentwicklung - in Zusammenarbeit mit den Ressorts - ein strategisches Monitoring in Bezug auf die Themen und Handlungsfelder der FTI-Strategie übernehmen.

IV.2.4 Die vielfältigen Maßnahmen der FTI-Strategie setzen bei Maßnahmen an der Schnittstelle zum Bildungssystem an, verfolgen eine Stärkung der Forschungsstrukturen (Grundlagen und angewandte Forschung) mit Blickrichtung auf Exzellenz, nehmen

\footnotetext{
${ }^{31}$ Für eine detaillierte Darstellung aller durch den EFS finanzierten Maßnahmen und unterstützten Zielgruppen wird für das Burgenland auf das Programm „Phasing Out Burgenland 2007-2013 - EFS“ (www.phasing-out.at) und für alle anderen österreichischen Bundesländer auf das Programm „Beschäftigung Österreich 2077-2013“ (www.esf.at) verwiesen.

${ }_{32}$ Potenziale ausschöpfen, Dynamik steigern, Zukunft schaffen. Der Weg zum Innovation Leader. Strategie der Bundesregierung für Forschung, Entwicklung und Innovation, März 2011 (http://www.bmwf.gv.at/fileadmin/user upload/Broschuere zur FTI-Strategie 01.pdf) ${ }^{33}$ Aktuelle Information in englischer Sprache über Österreich im Europäischen Forschungsraum und der Innovationsunion unter www.era.gv.at
} 
thematische Schwerpunktsetzungen vor und forcieren die Stärkung der Innovationskraft der Unternehmen.

\section{Maßnahmen im Rahmen des Euro-Plus-Pakts}

Auch hier ist die Strategie für Forschung, Technologie und Innovation (FTI) als zentrales Element anzuführen. Wichtige Schritte waren im Berichtszeitraum insbesondere die Einrichtung der Task Force FTI, die Erweiterung der Förderung im Rahmen des FWF auf indirekte Kosten, die Erhöhung der Forschungsprämie und die Erhöhung des Deckels für Auftragsforschung.

Im aktuellen Reformpaket (1. Stabilitätsgesetz 2012) sind hinsichtlich der Forschungsförderung durch die Forschungsprämie folgende Maßnahmen vorgesehen:

1. Anhebung des Forschungsdeckels bei der Auftragsforschung: Der bisherige Deckel in Höhe von $100.000 €$ bei Forschungsaufwendungen, die für externe Aufträge vergeben werden, wird auf 1 Mio. € angehoben. Bei einem Satz von 10\% bedeutet dies ab 2012 eine Erhöhung der maximalen Forschungsprämie von bisher $10.000 €$ auf $100.000 €$.

2. Einbeziehung der Forschungsförderungsgesellschaft $\mathrm{mbH}$ (FFG) bei der jahresbezogenen Geltendmachung einer Prämie: Zur Verbesserung der Inhaltsprüfung soll anlässlich der Geltendmachung einer Prämie für eigenbetriebliche Forschung ein Gutachten der FFG beizubringen sein, welches das Vorliegen der Voraussetzungen für eine Prämie dem Grunde nach beurteilt. Dieses Gutachten dient dem Finanzamt als Entscheidungsgrundlage im Prämienverfahren.

3. Erhöhte Rechtssicherheit durch Forschungsbestätigung und Feststellungsbescheid:

3.1 Forschungsbestätigung: Um dem Steuerpflichtigen insbesondere bei mehrjährigen Forschungsprojekten erhöhte Rechtssicherheit in Bezug auf die jährlich geltend zu machende Forschungsprämie zu geben, wird die Möglichkeit geschaffen, vom Finanzamt eine bescheidmäßige Bestätigung darüber zu erhalten, dass ein bestimmtes Forschungsprojekt die Voraussetzungen für eine Prämie dem Grunde nach erfüllt Forschungsbestätigung gemäß $\S 118 \mathrm{a}$ BAO). Dazu bedarf es ebenfalls eines Gutachtens der FFG, welches das Vorliegen der Voraussetzungen einer Forschung und experimentellen Entwicklung bestätigt.

3.2 Feststellungsbescheid über die jährliche Bemessungsgrundlage: ein Steuerpflichtiger kann sich vom Finanzamt die Richtigkeit der Bemessungsgrundlage für die Prämie bestätigen lassen. Dazu bedarf es einer diesbezüglichen Bestätigung des Wirtschaftsprüfers. Folgt das Finanzamt der Bestätigung, erlässt es einen - auf das jeweilige Wirtschaftsjahr bezogene - Feststellungsbescheid über die Höhe der Bemessungsgrundlag für die Forschungsprämie (gemäß § 108c ABs- 8 EStG). Forschungsbestätigungen und Feststellungsbescheide können ab dem 1. Jänner 2013 beantragt werden.

Bereits mit Budgetbegleitgesetz 2011 wurde die Forschungsprämie von 8\% auf 10\% erhöht. Um die Rahmenbedingungen für exzellente Forschungsgruppen zu verbessern und die wettbewerbsorientierte Forschung an Universitäten zu stärken wurde der Fonds zur Förderung der wissenschaftlichen Forschung (FWF), Österreichs zentrale Einrichtung zur Förderung der Grundlagenforschung, ab 2011 in die Lage versetzt, 20\% der Overheadkosten auf alle vom FWF geförderten Einzelprojekte und auf Projekte des Programms zur Entwicklung und Erschließung der Künste (PEEK) zu finanzieren. 


\section{Stärkung der Innovationskraft der Unternehmen}

IV.2.5 Das Innovation Union Scoreboard 2011 weist Österreich weiterhin einen ausgezeichneten Platz bei seiner Innovationsentwicklung zu. Um allerdings wieder zu den schon erreichten Niveaus und in weiterer Folge zu den Spitzenländern aufzuschließen werden die im Nationalen Reformprogramm 2011 angesprochenen Maßnahmen auch 2012 verstärkt fortgesetzt und neue Initiativen ergriffen. Das bedeutet, dass der Fokus darauf gelegt wird, die Innovationskraft und damit das Wachstumspotenzial der Unternehmen zu erhöhen.

\section{$\underline{\text { Neue Initiativen und geplante Maßnahmen }}$}

IV.2.6 Mit dem neuen Programm „Forschungskompetenzen für die Wirtschaft", sollen durch gezielte strukturelle Fördermaßnahmen die Unternehmen im systematischen Aufbau und der Höherqualifizierung des vorhandenen Forschungs- und Innovationspersonals unterstützt werden. Fokussiert wird dabei auf kleine und mittlere Unternehmen (KMU). Weiters soll das Programm für die Zusammenarbeit zwischen Unternehmen und tertiären Forschungseinrichtungen unterstützen und $\mathrm{zu}$ einer stärkeren Verankerung unternehmensrelevanter Forschungsschwerpunkte führen. Die drei Programmlinien sind Qualifizierungsseminare, Qualifizierungsnetze und Lehrveranstaltungen mit tertiärem Charakter.

IV.2.7 Die im Juni 2011 gestartete FTI-Initiative Intelligente Produktion hat das Ziel die Wettbewerbsfähigkeit und Nachhaltigkeit der österreichischen Industrie zu stärken um auf internationalen Märkten weiterhin zu bestehen. Die erforderlichen Forschungskompetenzen im Bereich der Produktionsforschung werden aufgebaut und vorhandene Produktionsstrukturen werden gestärkt. Industrie und Forschung kooperieren in gemeinsamen anwendungsorientierten Forschungsprojekten. Das Programm ist langfristig angelegt.

IV.2.8 Innovation fördern und öffentliche Mittel effizient einsetzen - dieses Ziel verfolgt das Leitkonzept einer innovationsfördernden öffentlichen Beschaffung(ÖIB), dessen Erarbeitung 2011 vom Ministerrat beschlossen wurde. In Zukunft wird die öffentliche Hand als Kunde vermehrt innovative Produkte und Dienstleistungen nachfragen, dadurch deren Entwicklung in Österreich unterstützen und damit gleichzeitig öffentliche Dienstleistungen und Infrastrukturen verbessern sowie langfristig Energie- und Verwaltungskosten einsparen. Die bisher erarbeiteten Maßnahmenvorschläge reichen von der Erstellung von Beschaffungsbudgets für IÖB und Berücksichtigung von IÖB in Strategieplänen, der Einrichtung einer Servicestelle, der Durchführung von konkreten (Pilot)Projekten, einem strukturierten Informationsaustausch zwischen Bedarfsträgern, Beschaffern und Anbietern (Unternehmen, vor allem KMU) über eine Novellierung des Bundesvergabegesetzes bis zu regelmäßigen Awareness- und Stakeholdertreffen. Zwischenzeitlich wurden bereits konkrete Umsetzungsmaßnahmen gesetzt bzw. solche eingeleitet, so wurde im Oktober 2011 in Österreich eine Pilotausschreibung für Verkehrsinfrastrukturforschung gestartet, bei der das Instrument der vorkommerziellen Beschaffung (PCP) erstmals zum Einsatz gelangte (Budgetvolumen der PCP-Ausschreibung: 2 Mio. €). Die Burghauptmannschaft startet mit Unterstützung des Bundes ein PCP-Pilotprojekt zum Thema „innovative Kälte/WärmeLösungen in historischen Gebäuden.

IV.2.9 Um Jugendliche im Alter zwischen 15 und 20 für Forschung und Entwicklung zu begeistern bzw. interessieren und ihre Kreativität zu wecken wurde der Wettbewerb Jugend Innovativ konzipiert, der bereits erfolgreich seit vielen Jahren bundesweit durchgeführt wird und immer wieder an die gesellschaftspolitischen Änderungen und Herausforderungen angepasst wurde. So gibt es heute spezielle Kategorien wie Klimaschutz und IKT neben den herkömmlichen wie Business, Design, Engineering und Science, wo z.B. zu technischen, 
sozialen, oder wirtschaftlichen Problemstellungen von den Jugendlichen im Rahmen einer schriftlichen Projektarbeit innovative Lösungsvorschläge erarbeitet und von einer Expertenbzw. Expertinnenjury bewertet werden.

IV.2.10 Einen Beitrag zur Verbesserung des Zugangs zu Informationen über Einrichtungen, die F\&E betreiben sowie zur Erleichterung einer Kontaktaufnahme mit AkteurInnen in Wissenschaft und Forschung stellt die geplante „Österreichische Forschungsstättenevidenz" dar, die bis spätestens Ende 2. Quartal 2012 auf der Homepage der Statistik Austria öffentlich zugänglich sein wird. Die „Österreichische Forschungsstättenevidenz" ist eine Web-Version des 1994 zuletzt in Papierform erschienen Forschungsstättenkatalogs. In diesem finden sich alle F\&E betreibenden Einrichtungen, die im Zuge der alle zwei Jahre stattfindenden F\&E-Statistikvollerhebungen ihr Einverständnis für eine Veröffentlichung gegeben haben. Der aktuelle Datenstand beruht auf der F\&EErhebung 2009 und umfasst rund 2.990 Eintragungen. Die nächste Aktualisierung ist für 2013 auf Basis der Daten der F\&E-Erhebung 2011 geplant.

IV.2.11 Als weiteres wichtiges Zeichen erfolgreichen Wissens- und Technologietransfers von öffentlichen Forschungseinrichtungen in die Wirtschaft werden herausragende Verwertungs-Spin-Offs mit dem Preis „Phönix 2012“ ausgezeichnet. Prämiert werden junge Verwertungs-Spin Offs, innovative UnternehmerInnen und NewcomerInnen mit besonders aktuellen und innovativen Themen.

IV.2.12 Die Energiesysteme und Netze werden in Zukunft erhöhte Anforderungen zu erfüllen haben. Dazu gehört die Integration eines steigenden Anteils erneuerbarer Energien aus dezentralen Quellen, auch mit zunehmend fluktuierender Aufkommenscharakteristik, ebenso wie die Vorbereitung auf eine zunehmende Durchdringung mit Elektromobilität.

IV.2.13 Das Programm Leuchttürme der Elektromobilität ist in Österreich zentral für die systemische Entwicklung und Umsetzung von E-Mobilitätslösungen im Spannungsdreieck Nutzer-Fahrzeug-Infrastruktur. 2012 fokussieren diese verstärkt auf die bedarfsgerechte und systemintegrative Implementierung eines international interoperablen E-Mobilitätsangebotes, auf Basis erster funktionsfähiger prototypischer Lösungsbausteine in Fahrzeugen und Infrastrukturen. Organisatorische Innovationen und technologische Lösungen sollen dabei ihre Praxistauglichkeit im Alltag innerhalb und außerhalb Österreichs beweisen, um im Sinne der österreichischen Industrielandschaft zur Stärkung der Zulieferindustrie und damit zur Arbeitsplatzsicherung und dem Arbeitsplatzausbau beizutragen.

IV.2.14 Im Mittelpunkt der Initiative Smart Grids steht die Entwicklung aktiver, bidirektionaler Elektrizitäts-Verteilnetze. Entsprechende Lösungen werden entwickelt, im Rahmen von Demonstrationsprojekten erprobt und validiert. Darüber hinaus werden im Rahmen einer Begleitforschung relevante Aspekte der Sicherheit und Resilienz, des institutionellen und rechtlichen Rahmens und der Wirtschaftlichkeit für die mittelfristige Einführung der neuen Systemlösungen analysiert. Die Finanzierung entsprechender Forschungs- und Demonstrationsprojekte erfolgt u.a. über den Klima- und Energiefonds. Für 2012/2013 werden die ersten Pilot- und Demonstrationsvorhaben in österreichischen Modellregionen umgesetzt sein und vorgestellt werden.

IV.2.15 Die internationale Kooperation von Clustern hat sich als wichtiger Hebel für die Wirkung der Cluster, insbesondere bei der Unterstützung der transnationalen Kooperationen von Unternehmen, besonders KMU, sehr bewährt. Das Programm „ClusterInternationalisierung" unterstützt daher die internationale Vernetzung von österreichischen Clustern.

IV.2.16 Entscheidend zur Umsetzung der FTI-Strategie ist auch die Frage, wie effizient, transparent und effektiv öffentliche Fördermittel vergeben werden. 2011 wurde daher mit Nachdruck an der Vereinfachung und Standardisierung der direkten Forschungsförderung 
gearbeitet. Dazu wurde einerseits ein Themenmanagement eingeführt, das heißt, dass zur Umsetzung bestimmter inhaltlicher Schwerpunkte alle passenden Instrumente herangezogen werden: Neben der direkten FTI-Förderung wurde etwa auch die Bedeutung von Regulierung, öffentlicher Beschaffung oder fiskalische Maßnahmen berücksichtigt. Daneben wurden durch die Einführung von Qualitätsmanagement und Förderportfoliomanagement Förderangebote neu strukturiert und harmonisiert. Über einen effizienteren Mitteleinsatz hinaus ergeben sich dadurch auch für Fördernehmer zahlreiche konkrete Verbesserungen. Diese Reformen stellten sich in einer von gegenseitigem Austausch und voneinander Lernen geprägten Atmosphäre in weiterer Folge auch für andere Einrichtungen als nachahmenswertes best practice Beispiel heraus. Konkret nützen bereits jetzt auch andere Ressorts dieses standardisierte Instrumentenportfolio bei ihren Förderaktivitäten.

\section{Strategische Ausrichtung und Umsetzung bestehender Maßnahmen}

IV.2.17 Zur Stärkung universitärer Transfer- und Verwertungsstrukturen gemäß der IPRecommendation der Europäischen Kommission wurde eine nationale Kontaktstelle (NCP) für IP-Angelegenheiten zur Koordinierung der nationalen Wissenstransfertätigkeiten und Abstimmung mit Einrichtungen in anderen Mitgliedstaaten eingerichtet.

IV.2.18 Zur Stärkung öffentlicher Forschungseinrichtungen werden im Rahmen der nationalen Kontaktstelle (NCP, siehe oben) Maßnahmen zur Vereinheitlichung von Wissensschutz (insbesondere IPAG - Intellectual Property Agreement Guide der österreichischen Universitäten zur Erarbeitung von Vertragsmustern, Ausbildungsmaßnahmen, Workshops) durchgeführt.

IV.2.19 Um die Forschungs- und Innovationsbasis von Unternehmen auszuweiten wurde das bereits etablierte Innovationsscheck-Programm im Juni 2011 um das Programm Innovationsscheck Plus erweitert. Der Innovationsscheck Plus ist ein Förderprogramm für Klein- und Mittelunternehmen in Österreich, die ihre Forschungs- und Innovationsleistung noch weiter vertiefen und vom Know-how der Forschungseinrichtungen profitieren wollen. Mit dem Innovationsscheck Plus können sich die Unternehmen an Forschungseinrichtungen (außeruniversitäre Forschungseinrichtungen, Fachhochschulen und Universitäten) wenden und je nach Bedarf deren förderbare Leistungen bis zu einer Höhe von maximal $12.500 €$ nach Zahlung eines 20\%igen Selbstbehalts mit dem Scheck in der Höhe von $10.000 €$ (Förderintensität 80\%) bezahlen.

IV.2.20 Ein von allen internationalen Vergleichen, nicht zuletzt dem Innovation Union Scoreboard, untermauertes Manko des österreichischen Innovationssystems ist die mangelnde Verfügbarkeit von Risikokapital, speziell für Unternehmen in der Frühphase. Im Jahr 2011 wurden deshalb mehrere Venture Capital Initiativen (VC-Initiative, CleantechFonds) der öffentlichen Hand gestartet, die als Fund-in-Fund Modelle verstärkt Anreiz für private Investoren bieten. Öffentliche Mittel in Höhe von mehr als 20 Mio. $€$ und privates Kapital in mindestens derselben Höhe werden in den nächsten $2-3$ Jahren dabei in junge innovative Unternehmen investiert.

IV.2.21 Das Seedfinancing Programm wird nunmehr als ein horizontales Programm durchgeführt (Schwerpunkt LISA - Life Science Austria - wird wie andere thematische Schwerpunkte integriert, wobei gemeinsam die Vorgründung und Management auf Zeit als Unternehmen von der aws angeboten wird.

IV.2.22 Die Dienstleistungsinitiative (DL-I) dient der Förderung von innovativen Dienstleistungsprojekten. Ziel der Dienstleistungsinitiative ist es, durch verstärkte Förderung von Dienstleistungsinnovationen, die Produktivität, Wertschöpfung und Exporte von Dienstleistungsunternehmen sowie von jenen Unternehmen in produzierenden Sektoren, die sich mit produktbegleitenden Dienstleistungen beschäftigen, zu erhöhen. 


\section{Stärkung der Forschung}

IV.2.23 Im Rahmen der FTI-Strategie des Bundes verfolgt die österreichische Bundesregierung das Ziel, die Rahmenbedingungen für exzellente Forschungsgruppen an Hochschulen und außeruniversitären Forschungseinrichtungen so auszugestalten, dass sie im globalen Wettbewerb mithalten können. Die Bundesregierung hatte bereits im Zuge des Budgets 2011 für den Aktionszeitraum 2011-2014 zusätzliche Offensivmittel beschlossen (insgesamt 720 Mio. €), die vor allem in FTI-Unterstützung in Form einer Erhöhung der Forschungsprämie von $8 \%$ auf $10 \%$, in die Stärkung der Universitäten und zusätzliche F\&EMaßnahmen (vor allem KMU) fließen. Diese Offensivmaßnahmen werden fortgeführt, um den Wirtschaftsstandort Österreich zu stärken. ${ }^{34}$

\section{$\underline{\text { Neue Initiativen und geplante Maßnahmen }}$}

IV.2.24 Zur Vernetzung von Schulen mit universitären und außeruniversitären Forschungseinrichtungen wurden zwei Initiativen gestartet. Einerseits wurde im November 2011 durch Young Science beim OeAD ein Informations- und Service-Zentrum als OneStop-Shop eingerichtet. Dessen Ziel ist es, durch die Bündelung Informationsangebot Synergien zwischen den einschlägigen Initiativen zu stärken und die Kooperationen mit dem BMUKK im Bereich der verbesserten Studienwahlberatung und der frühen Nachwuchsförderung auszubauen. Zusätzlich wird ab 2012 ein neues Gütesiegel an Schulen vergeben, die als Forschungspartner kontinuierlich und strukturiert mit wissenschaftlichen Einrichtungen zusammenarbeiten. Andererseits werden im Rahmen von Sparkling Science wissenschaftliche Projekte gefördert, die Schülerinnen und Schüler aktiv in den Forschungsprozess einbeziehen und nachhaltige Beiträge zur Verbesserung der Schnittstelle zwischen Schulen und Hochschulen beitragen.

\section{Strategische Ausrichtung und Umsetzung bestehender Maßnahmen}

IV.2.25 Das Austrian Institute of Technology (AIT) wurde umfassend saniert und reformiert, um es zum führenden High Tech Forschungszentrum Österreichs weiterzuentwickeln. Mit der Zukunftspartnerschaft zwischen Industrie und öffentlicher Hand, die im November 2011 eingegangen wurde, wird privates Innovationspotenzial angeregt und gemeinsam an den großen Forschungsfragen der Gegenwart (Grand Challenges) wie Energiezukunft, Mobilität oder der Stadt der Zukunft geforscht.

IV.2.26 Um die institutionelle Stärkung der Forschung in Österreich voranzutreiben sowie die Exzellenz zu erhöhen, wird das Institut of Science and Technology Austria (IST Austria) auf- und ausgebaut. Derzeit arbeiten 207 Personen (Vollzeitäquivalente) am Campus. Bis dato konnten 8 vom Europäischen Forschungsrat vergebene Förderungen (sogenannte ERC Grants) eingeworben werden. Das IST Austria wurde 2011 international evaluiert und das Gesamturteil lautete, dass das IST Austria eine neue Institution am Wege zur weltweit anerkannten Exzellenz ist. Dem Institut ist es bereits gelungen, zu einem bemerkenswerten Ort für interdisziplinäre Forschungsaktivitäten an der Schnittstelle von Computerwissenschaften, Neurobiologie sowie Molekular- und Zellbiologie zu werden.

IV.2.27 Mit dem Aufbau der Vision 2020 - Vienna Biocenter sowie dem Ausbau des Instituts für Molekulare Biotechnologie de Akademie der Wissenschaften (IMBA) am selben Standort setzt Österreich Impulse zur Stärkung und Verbesserung der Forschungsinfrastruktur unter vertiefter Einbeziehung postgradualer Ausbildung (VIPS) im Bereich Life Sciences. Gleichzeitig adressiert Österreich damit große gesellschaftliche Herausforderungen und setzt die erfolgreiche Cluster-Politik in diesem Bereich fort.

\footnotetext{
${ }^{34}$ siehe auch Österreichisches Stabilitätsprogramm. Fortführung für die Jahre 2011-2016, Bundesministerium für Finanzen, April 2012
} 
IV.2.28 Gemeinsame Nutzung von Infrastruktur. Im Rahmen der Campus Support Facility (CSF), erfolgt die Anschaffung von Großgeräten und Services am Standort Campus Vienna Biocenter zur gemeinsamen Nutzung für alle Institutionen inklusive der Garantie des Betriebs durch qualifiziertes Personal auf höchstem wissenschaftlichem Standard. Für vier Einheiten wurden bereits Infrastrukturinvestitionen getätigt bzw. vorhandene Infrastruktur in die Campus Support Facility $\mathrm{GmbH}$ überführt. Diese Einheiten haben bereits seit Mitte 2011 bzw. nun mit Beginn 2012 den Betrieb aufgenommen.

IV.2.29 Am selben Standort entsteht im Rahmen des IMBA ein österreichisches Competence Centre for Disease Modeling. Mit diesem Kompetenzzentrum wird das vorhandene Know-how am Institut gestärkt und die funktionale Genomforschung verbreitert. Das IMBA ist eine 100\% Tochtergesellschaft der Österreichischen Akademie der Wissenschaften (ÖAW) und mit 160 MitarbeiterInnen das größte ÖAW-Institut. Mit der Etablierung des Competence Centre for Disease Modeling wird das Center im Endausbau über 30-40 MitarbeiterInnen verfügen.

IV.2.30 Strategie ACR+ der kooperativen Forschungseinrichtungen: Das Grundkonzept von ACR+ ist eine Stärkung des Verbandes ACR (Austrian Cooperative Research) durch interne Maßnahmen, die zu mehr Kooperation, Nutzung gemeinsamer Ressourcen und Verbesserung des Dienstleistungsangebotes (vor allem F\&E) führen sollen. Damit sollen einerseits KMU durch das verbesserte Dienstleistungsportfolio einen kompetenten Partner für FTE-Projekte erhalten und andererseits ACR-Institute selbst durch die verstärkte Zusammenarbeit Synergien erzielen, die Mittel für andere Tätigkeiten im Bereich FTI frei machen. Weiters sollen auch gemeinsame Infrastrukturinvestitionen zur Verbesserung der Dienstleistung und zu einem effizienteren Mitteleinsatz führen.

\section{IV.3 Klimaschutz und Energie}

IV.3.1 Im Kontext der nationalen Klimaschutz- und Energieziele werden die Maßnahmen in den vier Bereichen (i) Forcierung der Erneuerbaren Energien, (ii) Reduktion der Treibhausgasemissionen, (iii) Erhöhung der Energieeffizienz und (iv) Effizienter Umgang mit natürlichen Ressourcen - Ressourceneffizienz mit Nachdruck umgesetzt. ${ }^{35}$ Damit soll der Übergang zu einer ressourceneffizienten, nachhaltigen und resilienten Wirtschaft beschleunigt und die konsequente Verfolgung eines qualitativen Wachstumspfades sichergestellt werden.

Meilensteinprojekte und neue Initiativen

- Ökostromgesetz (ÖSG) 2012

- Förderoffensive Thermische Sanierung: 100 Mio. € jährlich. 2011 - 2016

- 2. Nationaler Energieeffizienzaktionsplan

- Klimaschutzgesetz 2011

- Klimaschutzinitiative klima:aktiv

- Klima- und Energiefonds

- Ressourceneffizienz Aktionsplan (REAP)

- Stakeholder-Initiative „Wachstum im Wandel“

\section{Forcierung der Erneuerbaren Energien}

IV.3.2 Gemäß Richtlinie des Europäischen Parlaments und des Rates vom 23. April 2009 zur Förderung der Nutzung von Energie aus erneuerbaren Quellen (2009/EG) hat Österreich seinen Anteil für erneuerbare Energie am Bruttoendenergieverbrauch bis 2020 auf 34\% zu

${ }^{35}$ cf. Europa 2020. Österreichisches Reformprogramm 2011 (April 2011), pp. $20 \mathrm{ff}$ 
erhöhen. Entsprechend der Berechnungsmethode dieser Richtlinie betrug der Anteil im Basisjahr 2005 24,84\% und im Jahr 2010 bereits 30,8\%. Im Juli 2011 wurde das neue Ökostromgesetz (ÖSG) 2012 verlautbart. Es bezweckt unter anderem, dass durch technologiepolitische Schwerpunktsetzung neue Technologien zur Ökostromerzeugung die Marktreife erlangen. Die meisten Bestimmungen treten mit 1. Juli 2012 in Kraft. Mit der ÖSGNovelle 2009 und der Ökostromverordnung 2010 wurden die Ökostromtarife angehoben, was vor allem im Bereich Windkraft und Photovoltaik zu einem starken Ausbau der Technologien führte. Im neuen ÖSG 2012 wird für Windkraft-Anlagen ein Einmalbetrag von 80 Mio. $€$ und für Photovoltaik-Anlagen ein Betrag von 28 Mio. $€$ zur Verfügung gestellt. Windkraftanlagen, die nach der Warteliste einen Vertrag im Jahr 2012 oder 2013 erhalten hätten, bekommen sofort einen Vertrag zum Tarif von 9,7 Cent/kWh, für Kontrahierungen die sonst im Jahr 2014 oder später stattfinden würden sind 9,5 Cent /kWh vorgesehen. Für Photovoltaikanlagen besteht eine ähnliche Regelung, die Abschläge - nach Zeitpunkt der Kontrahierung und Höhe des beantragten Tarifs - von 2,5\% bis 22,5\% vorsieht. Die Bestimmungen betreffend Abbau des Projektrückstaus im ÖSG 2012 traten bereits am 30.7.2011 in Kraft.

Abgesehen von Maßnahmen zum Abbau der bestehenden Wartelisten durch die genannten Einmal-Budgets werden die Kontingente der jährlichen Unterstützungsvolumina erhöht. Der jährliche Unterstützungsbetrag für neu zu kontrahierende Ökostromanlagen, der sich innerhalb der ersten 10 Jahre jährlich um 1 Mio. € reduziert, beträgt im Jahr 201350 Mio. € davon entfallen:

$\nabla \quad 8$ Mio. $€$ auf Photovoltaik

$\nabla 10$ Mio. $€$ auf feste und flüssige Biomasse sowie Biogas (3 Mio. $€$ davon für feste Biomasse mit einer Engpassleistung bis $500 \mathrm{~kW}$ )

$\nabla$ mindestens 11,5 Mio. $€$ auf Windkraft

$\nabla$ mindestens1,5 Mio. $€$ auf Kleinwasserkraft

$\nabla 19$ Mio. $€$ auf den Resttopf (Wind, Wasserkraft, Photovoltaik-Netzparität)

Durch die neue Aufteilung in Unterkategorien soll verhindert werden, dass sich - wie in der Vergangenheit - einzelne Technologien stärker entwickeln als andere. Solange die oben genannten Kontingente nicht ausgeschöpft sind, gilt für Kontrahierungsanträge aufgrund des ÖSG 2012 weiterhin eine Kontrahierungspflicht. Die für jedes Kalenderjahr gesondert zu bestimmenden Tarife können mittels ein- oder mehrjähriger Verordnung festgelegt werden im zweitgenannten Fall ist nach Maßgabe der Kostenentwicklung eine Degression vorzusehen. Bis zum Inkrafttreten einer neuen Verordnung gelten die für das jeweilige Vorjahr gültigen Tarife mit einem Abschlag von $8 \%$ bei Photovoltaik, $1 \%$ bei Windkraft und $1 \%$ bei den sonstigen Ökostromtechnologien.

IV.3.3 Die EU-Richtlinie über erneuerbare Energieträger ${ }^{36}$ setzt unter anderem das Ziel, dass bis zum Jahr 2020 der Anteil an erneuerbarer Energie im Verkehr (vor allem Biokraftstoffe und Strom aus Erneuerbaren für den Verkehr auf der Schiene und Straße) 10\% betragen soll. Die Beimischung von Biokraftstoffen wird derzeit durch Steuerbegünstigungen bei der Mineralölsteuer unterstützt. Zur Forcierung der Reinverwendung werden Flottenumstellungen auf hohe Beimischraten mit mehr als 40\% Biodiesel und Biogas sowie Superethanol E85 und Pflanzenöl durch das klima:aktiv mobil Förderprogramm unterstützt. Die Substitution fossiler Treibstoffe mit biogenen Treibstoffen erreichte im Jahr 2010 einen energiebezogenen Anteil von 6,6\%. Beim Einsatz von Elektrizität im Verkehr (Bahn, Straßenbahn, etc.) betrug der anrechenbare Anteil erneuerbarer Energieträger 2010 65,3\%.

\footnotetext{
${ }^{36}$ cf. Richtlinie des Europäischen Parlaments und des Rates vom 23. April 2009 zur Förderung der Nutzung von Energie aus erneuerbaren Quellen (RL 2009/28/EG)
} 


\section{Reduktion der Treibhausgasemissionen}

IV.3.4 Österreich ist gemäß Entscheidung 406/2009/EG des Europäischen Parlaments und des Rates („Effort Sharing“-Entscheidung verpflichtet seine Treibhausgasemissionen in Sektoren, die nicht dem Emissionshandel unterliegen, bis 2020 um mindestens 16\% bezogen auf die Emissionen des Jahres 2005 zu reduzieren. Die größten sektoralen Verursacher von Treibhausgasen in Sektoren außerhalb des Emissionshandels sind in Österreich die Bereiche Verkehr, Industrie und produzierendes Gewerbe, Energie, Landwirtschaft sowie Raumwärme und Warmwasser in Gebäuden. Das Parlament hat im November 2011 ein Klimaschutzgesetz (KSG) beschlossen (BGBI. I Nr. 106/2011). Damit wurde ein Kernelement des Regierungsprogramms im Bereich der Umweltpolitik umgesetzt. Das Klimaschutzgesetz sieht die nationale Umsetzung völkerrechtlicher und gemeinschaftsrechtlicher Zielvorgaben sowie die Zuweisung von Verantwortlichkeiten für die Zielerfüllung an Sektoren und Gebietskörperschaften vor. Für die Erarbeitung von Maßnahmenplänen sowie zum Zweck der Gestaltung der langfristigen Klimapolitik wurde zudem das hochrangige Nationale Klimaschutzkomitee geschaffen, in dem die relevanten Ministerien, die Bundesländer und Sozialpartner vertreten sind. Die verbindliche Aufteilung von Verantwortlichkeiten für die Umsetzung der Zielvorgabe für den Zeitraum 2013 bis 2020 erfolgt durch eine gesonderte Vereinbarung zwischen Bund und Ländern auf der Grundlage des $\$ 7$ des KSG („Klimaschutzverantwortlichkeitsmechanismus“). Ergänzend dazu bietet die Klimaschutzinitiative klima:aktiv Beratung, Bewusstseinsbildung, Ausbildungsangebote und Qualitätssicherung in den vier Schwerpunktbereichen Energieeffizienz, Bauen und Sanieren, Erneuerbare Energien und Mobilität (klima:aktiv mobil).

IV.3.5 Zu den bereits bestehenden Maßnahmen wie Ausbau der Infrastruktur, Attraktivierung der öffentlichen Verkehrsmittel und klima:aktiv mobil mit den Schwerpunkten Fuhrparkumstellungen auf alternative Antriebe und Elektromobilität, klimaschonendes Mobilitätsmanagement sowie Radverkehrsförderung und Spritsparinitiative wird im Gebäudebereich die Förderung der thermisch-energetischen Sanierung von Wohngebäuden verfolgt sowie die Errichtung von Niedrigstenergie- und Passivhäusern im Neubau unter Einhaltung von energietechnischen Standards, welche in einer Vereinbarung zwischen Bund und Ländern nach Art. 15a-B-VG festgeschrieben sind. Darüber hinaus erfolgt ein jährliches Monitoring der $\mathrm{CO}_{2}$-Reduktionserfolge.

IV.3.6 Im Rahmen des Förderprogramms „Modellregion Elektromobilität“ konnte die Zahl der Modellregionen von 5 auf 8 erweitert werden. Neben Vorarlberg, Salzburg, Wien, Graz und Eisenstadt sind nun auch NÖ Wien-Süd, E-Logistik Großraum Wien und Klagenfurt dazugekommen. Der Schwerpunkt im Jahr 2012 liegt auf der Konsolidierung bestehender Modellregionen.

\section{Erhöhung der Energieeffizienz}

IV.3.7 Ein wichtiges Instrument für Wachstum und Wettbewerbsfähigkeit ist die Erhöhung der Energieeffizienz. Die Erhöhung der Energieeffizienz ist darüber hinaus eine wichtige Maßnahme zur Bekämpfung von Energiearmut, weil dadurch die Energiekosten nachhaltig gesenkt werden. Wie bereits im Reformprogramm 2011 dargelegt, sollen in Entsprechung der Entschließung des Nationalrates 182/E vom 7.7.2011 und in Umsetzung der Maßnahmenvorschläge für eine Energiestrategie in den kommenden Jahren Energieeffizienz- und Einsparmaßnahmen, insbesondere im Gebäude-, Verkehrs- und Haushaltsbereich umgesetzt werden. Im produzierenden Bereich werden Energiemanagementprogramme forciert und der Einsatz energieeffizienter Technologien und Prozesse gefördert. Ein weiterer wichtiger Schritt ist die Bewusstseinsbildung in Richtung energieeffizientes Verhalten. Daher werden Energieaudits und Energieberatung für Haushalte, Gemeinden und Betriebe unterstützt und diesbezügliche Informationskampagnen durchgeführt. Öffentliche Forschungsprogramme sollen die Umsetzung des Nationalen Energieeffizienzaktionsplans (inklusive der Weiterentwicklung des Monitoringsystems) 
unterstützen. Einen wichtigen Beitrag hierzu wird das derzeit in Bearbeitung befindliche Energieeffizienzpaket leisten. ${ }^{37}$ Zusammenfassend können aus den bisher umgesetzten Maßnahmen Endenergieeinsparungen in Höhe von $21,7 \mathrm{PJ}$ pro Jahr bis $2011^{38}$ hochgerechnet werden. Im Jahr 2010 liegt der Endenergieverbrauch bezogen auf die Wirtschaftsleistung um 4\% niedriger als 1995.

IV.3.8 Im Rahmen der Förderoffensive Thermische Sanierung sind für den Zeitraum 2011 bis 2016 vom Bund jährlich 100 Mio. € für die thermisch/energetische Sanierung privaten Wohnraums und betrieblich genutzten Gebäuden vorgesehen - dies zusätzlich zu den Maßnahmen im Rahmen der Wohnbauförderung der Länder (siehe IV.3.6). Für 2012 stehen als zusätzliche Bundesförderung 100 Mio. € zur Verfügung, davon 70 Mio. € für den privaten Wohnbau und 30 Mio. $€$ für Betriebsgebäude. Mit der Förderaktion 2011 wurden durch die Förderung der Sanierung von privatem Wohnraum und Betriebsgebäuden Investitionen in einer Größenordnung von rd. 860 Mio. € ausgelöst. Über 12.000 Arbeitsplätze wurden damit zum Teil gesichert bzw. zum Teil auch neu geschaffen. Mit den 2011 genehmigten Projekten wird auch ein beachtlicher Umwelteffekt erreicht - eine Einsparung(bei einer Nutzungsdauer der Investition von 30 Jahren) von insgesamt 4,4 Mio. Tonnen $\mathrm{CO}_{2}$. Drei Viertel davon entfallen auf private Haushalte, ein Viertel auf Betriebsgebäude. Diese Ziele werden mit der Förderaktion 2012 ebenfalls wieder angestrebt. Die Förderung der thermischen Sanierung trägt sowohl zum Treibhausgasziel als auch zur Erhöhung der Energieeffizienz bei und schafft einen Mehrwert für Wachstum und Beschäftigung.

IV.3.9 Im Rahmen der Umweltförderung im Inland (UFI) werden Maßnahmen im Bereich der Energieeffizienz und der Nutzung regenerativer Energieträger (ausgenommen Strom) im betrieblichen Bereich (Energiebereitstellung, Produktionsprozess, Gebäudeeffizienz, Mobilität) gesetzt. Für die Förderungen im Rahmen der UFI stehen in den Jahren 2011 bis 2014 jährlich rund 90 Mio. € zur Verfügung; auf Basis der bisherigen $\mathrm{CO}_{2}$-Einspareffekte wird damit ein jährlicher $\mathrm{CO}_{2}$-Einspareffekt von über 380.000 Tonnen, über die Nutzungsdauer 7,8 Mio. Tonnen erzielt. Zusätzlich werden mit den jährlichen finanziellen Mitteln für thermische Sanierungsmaßnahmen im Wohn- und betrieblichen Bereich in Höhe von 143.000 Tonnen $\mathrm{CO}_{2}$ pro Jahr (= 4,3 Mio. Tonnen über die Nutzungsdauer) bewirkt. Darüber hinaus sind mit diesen Maßnahmen erhebliche volkswirtschaftliche Effekte (insbesondere Beschäftigung, Wertschöpfung, Budgeteinnahmen) verbunden.

IV.3.10 F\&E im Bereich nachhaltige Energietechnologien und Klimaforschung wird über den Klima- und Energiefonds (KLIEN) gefördert. Förderungsschwerpunkt ist neben Energietechnologien und Klimaforschung die Nutzung von Energieeinsparungspotenzialen in Betrieben sowie die regionale Ebene. Im Zeitraum 2007-2011 hat der Klima- und Energiefonds insgesamt fast 600 Mio. $€$ an Förderzusagen und Forschungsaufträgen erteilt (ca. 150 Mio. € davon im Jahr 2011). Zur Realisierung einer nachhaltigen Energienutzung bis hin zum langfristigen Saldoziel der Energieautarkie spielen die Klima- und Energiemodellregionen eine wichtige Rolle.

Die derzeit bestehenden 85 Klima- und Energiemodellregionen, in denen ca. 2 Mio. Menschen leben umfassen 884 der 2.357 österreichischen Gemeinden.

IV.3.11 Im Rahmen des neu initiierten Programms Fit for SET-smart energy demo wird der Aufbau von geeigneten Konsortien und die Errichtung von Smart CitiesDemonstrationsprojekten gefördert. Das Ziel ist die $\mathrm{CO}_{2}$-neutrale Stadt, welche durch den Einsatz und der intelligenten Vernetzung neuer Energie- und Verkehrstechnologien entsteht. Gleichzeitig sollen sich österreichische Forschungsinstitutionen, Betriebe und Kommunen international vernetzen und damit "fit" für europäische Ausschreibungen zur Smart Cities Initiative des SET-Plans machen.

\footnotetext{
${ }^{37}$ Siehe dazu insbesondere auch „Zweiter Nationaler Energieeffizienzaktionsplan der Republik Österreich 2011“ (NEEAP)

${ }^{38}$ cf. 2. NEEAP
} 
IV.3.12 Mit der Novellierung der Normverbrauchsabgabe (NoVA) wurde ein weiterer Schritt in Richtung Ökologisierung gesetzt. Für verbrauchsarme $\left(\mathrm{CO}_{2}\right.$-Emissionen unter $\left.120 \mathrm{~g} / \mathrm{km}\right)$ neue Pkw und alternative Antriebe kann ein Steuerbonus beansprucht werden. Gleichzeitig wird ab 2013 die Malusbemessungsgrenze auf 150g/km, 170g/km bzw. 210g/km verschärft. 2011 wurden die Steuersätze für Benzin und Diesel erhöht, eine Flugabgabe neu eingeführt und die Energieabgabevergütung auf Produktionsbetriebe beschränkt.

\section{Effizienter Umgang mit natürlichen Ressourcen - Ressourceneffizienz}

IV.3.13 In Umsetzung des Schwerpunktes der Europa 2020 Strategie „Ein ressourceneffizientes Europa“ wurde im Jänner 2012 der nationale „Ressourceneffizienz Aktionsplan" (REAP) veröffentlicht. Langfristiges Ziel des Aktionsplans ist es, die österreichische Wirtschaftsentwicklung vom Ressourcenverbrauch und den damit einhergehenden Umweltauswirkungen zu entkoppeln. Der REAP legt mittel- und langfristig nationale Ziele zur Steigerung der Effizienz bei der Nutzung natürlicher Ressourcen fest, er identifiziert vier wesentliche Aktionsfelder (ressourceneffiziente Produktion, öffentliche Beschaffung, Kreislaufwirtschaft und Bewusstseinsbildung) und schlägt Instrumente und erste Maßnahmen für die Periode 2012-2013 für eine konkrete Steigerung der Ressourceneffizienz in Österreich vor. Der Österreichische Rohstoffplan unterstützt die verstärkte Wiederverwertung aus Alt- und Abfallstoffen, die Entwicklung von Methoden zur Reduktion des Materialeinsatzes, optimale Berücksichtigung mineralischer Rohstoffe in der Raumordnung.

IV.3.14 Mit der Überarbeitung der Österreichischen Nachhaltigkeitsstrategie des Bundes (geplanter Beschluss im Juni 2012) wird darüber hinaus der strategische Rahmen für nachhaltiges Wirtschaften geschaffen, der einen qualitativen Wachstumspfad voraussetzt und neben Politik, Verwaltung und Wirtschaft alle gesellschaftlichen Gruppen adressiert. Ergänzend dazu will die Stakeholder Initiative „Wachstum im Wandel“, die von über zwanzig Institutionen (Ministerien, Landesregierungen, Interessenvertretungen, NGOs) mitgetragen wird, einen breiten öffentlichen Diskurs zur Frage anregen, wie ein zukunftsfähiges Wirtschafts- und Finanzsystem gestaltet werden kann und wie Wohlstand und Lebensqualität in Zukunft gemessen werden sollen. In Workshops und Konferenzen werden gemeinsam Lösungsansätze erarbeitet (www.wachstumimwandel.at). Ein wichtiger Maßnahmenschwerpunkt ist die gesellschaftliche Verantwortung von Unternehmen (Corporate Social Responsibility) sowie die Forcierung nachhaltiger Unternehmensführung in der Praxis.

IV.3.15 In enger Zusammenarbeit mit der Wirtschaft wird über den Masterplan green jobs schwerpunktmäßig F\&E mit Hinblick auf ressourcen-effiziente und kohlenstoffarme Wirtschaft unterstützt sowie die Ausbildung von „Energiemanagern“. Derzeit gibt es in Österreich rund 210.000 green jobs, die im Zusammenhang mit Umweltschutz und erneuerbarer Energie stehen. Diese Arbeitsplätze stehen für 11,8\% des BIP. Das bedeutet, dass sowohl der Umsatz als auch die Beschäftigung in der Umweltwirtschaft im Vergleich zur Gesamtwirtschaft überproportional angestiegen ist und damit jedenfalls positiv zur Beschäftigungsentwicklung in Österreich beigetragen hat. Der Aktionsplan Nachhaltige Beschaffung (naBe) definiert ökologische Mindestanforderungen für nachhaltige öffentliche Beschaffung (BVerG) und der Masterplan Umwelttechnologie (MUT) verfolgt die Forcierung von Umwelttechnologien als Ziel. Über die Initiative Green Events Austria werden Umwelt- und Nachhaltigkeitsaspekte in besonderem Maße in die Gestaltung der Organisationsabläufe von Großprojekten verankert (z.B. Schi-WM Schladming 2013). Durch Maßnahmen im Bereich der Bewusstseinsbildung (z.B. Wettbewerbe) werden Unternehmen wie Einzelpersonen dazu motiviert, Veranstaltungen als "green event" zu organisieren und dadurch eine hohe Breitenwirksamkeit und Einspareffekte von $\mathrm{CO}_{2}$ und natürlichen Ressourcen erreicht. 


\section{IV.4 Bildung}

IV.4.1 Das Österreichische Reformprogramm 2011 nannte als die wichtigsten Herausforderungen in diesem Bereich die Steigerung der Bildungsbeteiligung, die Erhöhung der Anzahl der AbsolventInnen naturwissenschaftlich-technischer Studienrichtungen, die Verbesserung des Bildungsniveaus sowie die Senkung der Schulabbrecherlnnenquote, die Verbesserung der Attraktivität und Durchlässigkeit der beruflichen Bildung.

IV.4.2 Im Rahmen der länderspezifischen Empfehlung wird Österreich aufgefordert, Schritte zu unternehmen, um die Ergebnisse im Bildungsbereich zu verbessern und die Schulabbrüche zu verhindern.

\section{Meilensteinprojekte und neue Initiativen}

- Hochschulraum-Strukturmittel 450 Mio. € 2013 bis 2015

- Erhöhung des Globalbeitrags der Universitäten 300 Mio. € 2013 bis 2015

- Verbesserung der Lehr- und Studiensituation durch Fortführung der Offensivmittel: 240 Mio. € 2013 bis 2015 (z.B. MINT/Masse Offensive an Universitäten, Ausbau der Fachhochschulen, FWF-Overheads)

- Bekämpfung der Dropoutproblematik (Schwerpunkt Prävention)

- Ausbau ganztägiger Schulformen Im Rahmen der „Offensivmittel“ sind im Finanzrahmen 2013-2016 jährlich 80 Mio. € vorgesehen (Teil des Euro-Plus-Pakts)

- Neue Mittelschule (als Schule der Zukunft eine zusätzliche Investition in die Qualität des Unterrichts, in Individualisierung und Teamteaching)

- PädagogInnenbildung NEU (Erhöhung der Qualität der Lehrerlnnenausbildung)

- Nationale Strategie zum Lebenslangen Lernen (Umsetzung von in der „Europa 2020 Strategie" verankerten Maßnahme im Bildungsbereich)

- Die neue Reife- und Diplomprüfung (Sicherstellung der nationalen und internationalen Vergleichbarkeit von Schulabschlüssen)

- Nachholen von Bildungsabschlüssen (Wiedereinstieg ins Bildungssystem für bildungsferne Personen: 54,6 Mio. € bis 2014)

- Berufsorientierung und Bildungsberatung (Verbindlicher Maßnahmenkatalog in der 7. und 8. Schulstufe)

- Qualitätsinitiative Berufsbildung (QIBB): Entwicklung und dauerhafte Einführung eines umfassenden Qualitätsmanagementsystems im berufsbildenden Schulwesen)

- Lehre mit Matura (Anhebung des Bildungsniveaus von Lehrlingen und Verbesserung der Arbeitsmarktchancen für diese Zielgruppe

- Verpflichtendes kostenloses Kindergartenjahr für alle 5-jährigen

IV.4.3 Die österreichische Bundesregierung hat im Stabilitätspaket 2012-2016 sorgfältig darauf geachtet, dass der Spielraum für Zukunftsinvestitionen erhalten bleibt und die eingegangen Verpflichtungen im Rahmen des Euro-Plus-Paktes erfüllt werden. ${ }^{39}$

\footnotetext{
${ }^{39}$ siehe auch Österreichisches Stabilitätsprogramm. Fortschreibung für die Jahre 2011-2016, Bundesministerium für Finanzen, April 2012
} 


\section{Maßnahmen im Rahmen des Euro-Plus-Pakts}

\section{Österreichischer Hochschulplan}

Die übergeordnete Zielsetzung des Hochschulplans liegt darin, den österreichischen Hochschulraum besser abzustimmen und dadurch erfolgreich weiterzuentwickeln, höchste Qualität in Lehre und Forschung sicherzustellen und die internationale Sichtbarkeit zu erhöhen.

Teilprojekte des Hochschulplans:

1. Bauleitplan: Ziel ist eine klare und mit den Universitäten vor Ort abgestimmte Reihung künftiger Bauvorhaben und -notwendigkeiten, die dem vereinbarten Zeitplan gemäß abgearbeitet wird.

2. Forschungsinfrastruktur/Internationales: Mittels des Forschungsinfrastrukturplans wird bestimmt, wo künftig Großanlagen für die Grundlagenforschung errichtet werden. Ziel ist es, die internationale Wettbewerbsfähigkeit durch nationale Koordination und eine stärkere Profilierung durch Prioritätensetzung zu erreichen.

3. Koordinationsmaßnahmen: Ziele sind:

- Entwicklung und Organisation eines institutionalisierten Koordinierungsgremiums („Hochschulkonferenz") und entsprechender Mechanismen unter Involvierung relevanter Stakeholder

- Entwicklung von Koordinierungsmaßnahmen insbesondere auch für Lehre und Forschung

- Schaffung neuer Instrumente bzw. neuer Kommunikationskulturen zur Steuerung des Hochschulraums

4. Kapazitätsorientierte Studienplatzfinanzierung: Die Universitätsfinanzierung soll künftig auf die drei Säulen Lehre, Forschung bzw. Entwicklung und Erschließung der Künste sowie Infrastruktur aufgeteilt werden. Die "Studienplatzfinanzierung" ist als neue Universitätsfinanzierung zu sehen, die künftig insbesondere die Grundfinanzierung der Lehre der Universitäten sicherstellen soll. Dies bedingt $u$. a. eine Kapazitätenregelung unter Berücksichtigung einer Studierendenplatzzahlorientierung und ggf. einer Steigerung. Mit der schrittweisen Einführung der neuen Instrumente soll nach und nach ab der nächsten Leistungsvereinbarungsperiode (2013-2015) begonnen werden, so dass ein reibungsloser Übergang und eine Umstellung mit den Leistungsvereinbarungen ab 2015 möglich ist.

\section{Erhöhung der Anzahl der Fachhochschulplätze}

Die qualitative Weiterentwicklung des Fachhochschulsektors ist unter anderem im sogenannten Fachhochschulplan angeführt, der die inhaltlichen Schwerpunkte des FHSektors für die nächsten jeweils drei Jahre festlegt (2010/11 bis 2012/2013). Demnach sind unter anderem Schwerpunkte im Bereich Technik/Naturwissenschaften, Ausbau der berufsbegleitenden Studien, Ausbau der angewandten Forschung sowie Internationalisierung vorgesehen. Weiters wurden durch die Implementierung des Hochschul-Qualitätssicherungsgesetzes (HS-QSG) und der damit verbundenen Novellierung des Fachhochschul-Studiengesetzes (FHSTG) erweiterte Qualitätskriterien für den Fachhochschulsektor geschaffen. Durch die Fortführung der Offensivmittel werden am $\mathrm{FH}$ Sektor im Vollausbau zusätzlich ca. 4.000 Studienplätze geschaffen.

\section{Verbesserung der forschungsgeleiteten Lehr- und Studiensituation}

Zur Stärkung der Lehre zur Erhöhung der Qualität in der Hochschulbildung bei gleichzeitiger Effizienzsteigerung wurden zusätzliche Offensivmittel den Hochschulen zur Verfügung gestellt. Besondere Schwerpunkte liegen abgesehen vom Ausbau des FH-Sektors 
- bei der Verbesserung der Studiensituation in den sog. „Massenfächern“ und in der Stärkung der wichtigen MINT-Fächer (Mathematik, Informatik, Naturwissenschaften und Technik).

- bei der Neustrukturierung der Kooperation von Universitäten mit außeruniversitären Forschungseinrichtungen und

- dem Neustart der universitären "Overhead-Finanzierung“ im Rahmen der Forschungsfinanzierung durch den FWF.

\section{Ausbau ganztägiger Schulformen}

2006 war die Ausgangslage ein Angebot von 62.700 Betreuungsplätzen. Im Schuljahr 2011/12 sind es bereits 105.000 Plätze. In zumindest einer Schule pro Bezirk wird schulische Tagesbetreuung angeboten. Insgesamt gibt es bereits an 25\% der Schulen der Primar- und Sekundarstufe I Betreuungsangebot. Im Jahr 2012/13 soll es schon eine Verdoppelung gegenüber 2006 auf rund 120.000 Plätze geben.

Bis 2016 werden jährlich 80 Mio. € Bildungs-Offensivmittel investiert. Ziel ist es, dort ein Angebot an Tagesbetreuung zu schaffen, wo eine entsprechende Nachfrage besteht. Dafür werden die derzeit bestehenden 105.000 Plätze auf 160.000 Plätze für die schulische Tagesbetreuung ausgeweitet. Inklusive der Hortplätze sollen ab 2015 insgesamt 210.000 Plätze angeboten werden, was eine Verdoppelung des Angebots darstellt.

Im Parlament wurde das Paket zum Ausbau der Ganztägigen Schulformen beschlossen:

- Neben klassen-, schulstufen-, und schulübergreifenden Angeboten wird schulartenübergreifende Tagesbetreuung ermöglicht.

- Schon ab 12 angemeldeten Schülerlnnen - falls keine schulübergreifende Form der Ganztagesbetreuung möglich ist - muss ein verpflichtendes Angebot zu schulischen Tagesbetreuung zustande kommen.

- Für den Bereich der Freizeitpädagogik gibt es eine Ausbildung als „akademischer Freizeitpädagoge“ (2 Semester, berufsbegleitend) an Pädagogischen Hochschulen.

Mit den Bundesländern wurden 15a-Vereinbarungen abgeschlossen, mit denen die Zuteilung der Offensivmittel, die als Anschubfinanzierung und zum Teil für den Ausbau der Infrastruktur eingesetzt werden, geregelt wird. Dabei werden auch einheitliche Qualitätskriterien verankert. Ziel ist insbesondere auch eine verstärkte Kooperation mit Sport- und Kulturvereinen sowie Musikschulen.

\section{Steigerung der Bildungsbeteiligung, Vorbereitung auf das Studium und Erhöhung der Mobilität im tertiären Sektor}

IV.4.4 Die folgenden genannten Maßnahmen tragen zur Erhöhung des Anteils der HochschulabsolventInnen bzw. zu einer bewussten Studienwahl bei und sind mehrjährig konzipiert. Die Ergebnisse können erst zu einem späteren Zeitpunkt evaluiert werden.

\section{Strategische Ausrichtung und Umsetzung bestehender Maßnahmen}

IV.4.5 Im Bereich Hochschulbildung ist es wesentlich, die Qualität der Studienwahlberatung und damit eine bewusste Studienwahl zu fördern sowie einen Beitrag zu einer besseren Verteilung der Studierendenströme zu leisten. Das bestehende Studienberatungsangebot soll künftig erweitert werden. Der Studienchecker findet im Schuljahr 2011/12 an ca. 190 Schulen (AHS und BHS) statt und ist ab diesem Schuljahr in allen Bundesländern vertreten. Für die nächsten Jahre ist bis zum flächendeckenden Ausbau 2014/15 eine schrittweise Ausweitung vorgesehen. Die Studieninformation wird durch einen Relaunch der Website www.studienwahl.at maßgeblich verbessert. Seit 1. Dezember ist die überarbeitete Version online. 
IV.4.6 Um die Effektivität zu steigern, wird das Angebot zur Vorbereitung auf das Studium verbessert und im Rahmen der Studienberatung NEU verpflichtend konzipiert. Zunächst werden jedoch bereits begonnene Maßnahmen wie der Studienchecker und die Studienberatung für Präsenzdiener vertieft, Kooperationen mit der Österreichischen HochschülerInnenschaft ausgebaut sowie Möglichkeiten der Online-Beratung sondiert. Erst in der Folge - nicht vor 2015 - ist an eine verpflichtende Studienberatung gedacht. Diese Maßnahmen werden bereits jetzt begleitend evaluiert. Langfristig kann die Wirksamkeit (insbesondere des Programms Studienchecker) erst aufgrund einer begonnenen Langzeitstudie beurteilt werden.

IV.4.7 Um eine gesamthafte Koordinierung des österreichischen Hochschulraums auch zum Zweck der Effizienzsteigerung des Systems zu erreichen, wurden die Eckpunkte des Hochschulplans im Herbst 2011 erarbeitet. Die einzelnen Teilprojekte (Bauleitplan, Forschungsinfrastruktur/Internationales, Koordinationsmaßnahmen und „Kapazitätsorientierte Universitätsfinanzierung/Studienplatzfinanzierung) werden weiterentwickelt und laufend umgesetzt.

IV.4.8 In Österreich wird eine qualitative und quantitative Weiterentwicklung und Konsolidierung des Fachhochschulsektors durchgeführt. Durch zusätzliche Mittel werden an den Fachhochschuleinrichtungen neue Studienplätze geschaffen.

IV.4.9 Um die Mobilität im tertiären Sektor zu erhöhen werden verschiedene Maßnahmen fortgesetzt. Darunter Erasmus back to school. Alleine im vergangenen Studienjahr kehrten mehr als 80 Erasmus Studierende an ihre ehemaligen Schulen zurück, um dort von ihrem Erasmus-Aufenthalt zu erzählen. Mehr als 3.500 Oberstufen-Schülerlnnen haben damit wertvolle Informationen und praktische Tipps erhalten. Im Oktober 2011 startete die Fortsetzung des erfolgreichen Projektes.

IV.4.10 Für Österreich ist die Förderung der internationalen Studierendenmobilität im Rahmen der Mobilitätsprogramme ebenso ein besonderes Anliegen.

Durch die Nutzung der europäischen internationalen und nationalen Programme wie z.B: ERASMUS und CEEPUS wurde eine erneute gezielte Steigerung der Studierenden- und Graduiertenmobilität erreicht. Neben der Österreichischen Datenbank für Stipendien und Forschungsförderung www.grants.at ist die europaweite EURAXESS-Initiative EURAXESS - Researchers in Motion ein fixer Bestandteil der Informationstätigkeit für mobile Forschende.

\section{Anzahl der Absolventinnen und Absolventen naturwissenschaftlich- technischer Studienrichtungen}

\section{Strategische Ausrichtung und Umsetzung bestehender Maßnahmen}

IV.4.11 Mit Informationsoffensive zu MINT-Fächern sollen StudieninteressentInnen und StudienanfängerInnen besser über die diversen Studienmöglichkeiten in den Fachbereichen Mathematik, Informatik, Naturwissenschaften und Technik an Unis und Fachhochschulen informiert sowie deren Berührungsängste abgebaut und über ihre Jobchancen aufgeklärt werden. Mit zusätzlichen Mitteln für MINT- und Massenfächer werden Verbesserungen in der Lehre erzielt und MINT-Projekte finanziert und umgesetzt. In den Jahren 2011 und 2012 werden dafür zusätzliche Mittel in der Höhe von insgesamt 40 Mio. $€$ aufgewendet.

IV.4.12 Erweiterung der Programme zur Frühförderung des wissenschaftlichen Nachwuchses. Um ein ausreichendes Angebot an AbsolventInnen mathematischnaturwissenschaftlicher und technischer Studiengänge zu erreichen, wird gezielt die Vernetzung von Schulen mit universitären und außeruniversitären Forschungseinrichtungen und Unternehmen aufgebaut. Dazu wurde im November 2011 die Plattform „Young Science" vorgestellt, welche die Kooperation zwischen Schule und Hochschule stärken soll. 
Die Initiative Young Science umfasst unter anderem die Kinderuniversitäten, das Projekt rio+20 sowie das Programm Sparkling Science. Diese wurde aufgrund des hohen Wirkungsgrades der Förderinitiative im Laufe des Jahres 2011 weiter ausgebaut und wird in den kommenden Jahren fortgesetzt. Zusätzlich wird im Jahr 2012 erstmals ein Gütesiegel an jene Schulen vergeben, welche intensiv mit Forschungs- und Hochschuleinrichtungen zusammenarbeiten.

IV.4.13 IMST - „Innovationen Machen Schulen Top“ ist ein flexibles Unterstützungssystem zur Stärkung, Etablierung und strukturellen Verankerung der Innovationskultur in Mathematik, Informatik, Naturwissenschaften, Deutsch und Technik (MINDT) an österreichischen Schulen. Durch IMST wurden in den letzten Jahren wichtige Entwicklungsimpulse in der strukturellen Weiterentwicklung des Bildungssystems, aber auch inhaltlich im Bereich der Unterrichts- und Schulentwicklung gesetzt.

IV.4.14 Vor allem die Herstellung von Schnittstellen zwischen dem Bildungs- und dem Innovationssystem ist zentral, um Kindern und Jugendlichen die entscheidenden Kompetenzen für eine aktive Partizipation an der immer innovationsorientierteren Innovationsgesellschaft zu vermitteln. Im Rahmen der Initiative Talente Praktika werden Forschungspraktika für SchülerInnen gefördert. Alleine im Jahr 2011 wurden 1387 qualitativ hochwertige FTI-Praktika gefördert und ein Drittel $(31,6 \%)$ davon wurde von Mädchen absolviert. Die Förderung beträgt hier $1.000 €$ pro Praktikumsplatz und das Unternehmen entlohnt die PraktikantInnen mit mindestens $700 €$ brutto.

\section{Verbesserung des Bildungsniveaus und Senkung der Schulabbrecherinnen- und Schulabbrecherquote}

IV.4.15 Die Datenlage betreffend die Dropoutproblematik ergibt folgendes Bild: 8,3\% der 18bis 24-Jährigen verfügten 2010 entsprechend den Berechnungen zum EU-Benchmark zu Early School Leaving über keinen Abschluss der Sekundarstufe II bzw. einer Lehre und befanden sich zum Zeitpunkt der Erhebung in keiner Weiterbildung. Österreich hat das Kernziel $(9,5 \%)$ bezüglich des Schulabbruches bereits erreicht, es werden aber weiterhin wichtige Maßnahmen in diesem zentralen Bereich gesetzt.

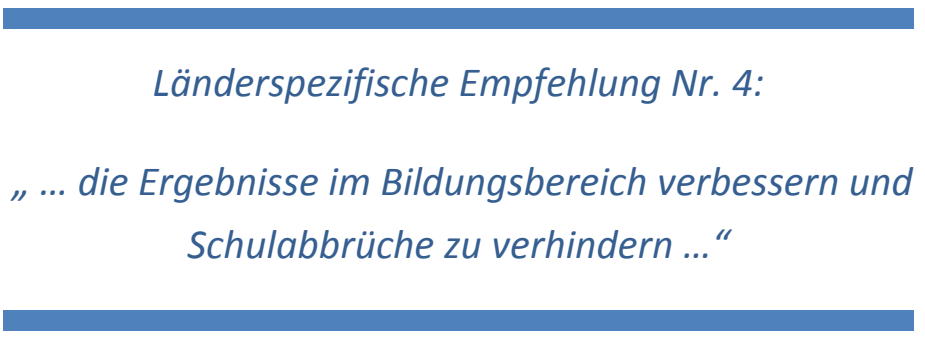
Berufsentscheidung" an alle 1.700 Schulleiter der AHS-Unterstufen, der Hauptschulen und der Allgemeinen Sonderschulen. Rundschreiben des BMUKK „Katalog verbindlicher Maßnahmen für die 7. Und 8. Schulstufe im Bereich Information, Beratung, Orientierung“ (schulische Schritte im Rahmen des Regelunterrichts und der verbindlichen Übung "Berufsorientierung“, verbindliches Ausmaß an Realbegegnungen (berufspraktische Tage, Betriebserkundigungen, Exkursionen), Besuch eines Berufsinformationszentrums, Verantwortung der Schulleitung - standortbezogenes Konzept, Reader zur Umsetzung (Informationen, Unterrichtsmaterialien und methodisches Hintergrundwissen) in Form eines Webportals.

IV.4.17 Oberstufe NEU: Individualisierung und gezielte Förderung: Seit mehreren Jahren gibt es Schulversuche an AHS- und BMHS-Standorten, deren Ziel eine effiziente 
Neugestaltung der Schullaufbahn ist. Seit diesem Schuljahr sind österreichweit ca. 40 Standorte beteiligt. Auf Basis der Erfahrungen wurde in einer Arbeitsgruppe des BMUKK ein Modell der Oberstufe NEU mit semesterweiser Lehrstoffverteilung (Kompetenzmodule) entwickelt. Im Zentrum stehen ein erweitertes Frühwarnsystem, individuelle Lernbegleitung und Fördermaßnahmen. Die semesterweise Lehrstoffverteilung bedeutet eine Verdichtung des Lern- und Arbeitsklimas und schafft einen besseren Überblick über etwaige Defizite von SchülerInnen. Ein erweitertes Frühwarnsystem mit konkreten Leistungsvereinbarungen wurde implementiert. Es geht um schrittweise und kontinuierliche Leistungserbringung und die verbesserte Vorbereitung auf eine universitäre Ausbildung. Es geht um das Fördern von Selbständigkeit und Eigenverantwortung und das Eingehen auf Interessen und Begabungen.

\section{Strategische Ausrichtung und Umsetzung bestehender Maßnahmen}

IV.4.18 Entsprechend den internationalen Empfehlungen (OECD, EU) sind das Hinausschieben der ersten Bildungswegentscheidung sowie die Individualisierung des Lernens von zentraler Bedeutung für die Verhinderung von frühzeitigem Schulabbruch.

IV.4.19 Der Ausbau der Neuen Mittelschule zur Regelschule ist ein zentrales Reformprojekt. Der Strukturwandel wird von einer Qualitätsoffensive begleitet, deren Ziel eine moderne Leistungsschule mit einer neuen Lernkultur ist. Eine entsprechende Novelle befindet sich derzeit in parlamentarischer Behandlung. Erstmals seit 50 Jahren wird mit der Neuen Mittelschule ein neuer Schultyp flächendeckend ins Regelschulwesen übernommen. Die Neue Mittelschule wird die Schülerinnen und Schüler je nach Interesse und Neigung, Begabung und Fähigkeit zum Besuch einer mittleren oder höheren Schule befähigen und sie auf das Berufsleben vorbereiten. In diese Reform werden unter anderem für die individuelle Förderung durch Team Teaching in den Pflichtgegenständen Deutsch, Mathematik und lebende Fremdsprache im Vollausbau rund 230 Mio. € pro Jahr investiert. Durch die sechs zusätzlichen Stunden pro Woche für die Individualisierung entstehen rund 4.000 neue Arbeitsplätze für Lehrerinnen und Lehrer.

Die Kernpunkte des Reformprojekts, etwa ein Lehrplan, der in der Vertiefung dem der AHS entspricht, klare Berechtigungen beim Übertritt in eine höhere Schule und vor allem eine neue Lehr- und Lernkultur durch Individualisierung, sowie gezielte Förderung bei Schwächen und bei Stärken werden umgesetzt. Ebenso wird es jeder Schule möglich sein, autonom eigene Schwerpunkte zu setzen. Für die Entwicklungsarbeit stehen Lerndesigner zur Verfügung. Aus dem Lehrer als Wissensvermittler wird der Lehrer als Lernbegleiter. Auch weitere AHS-Unterstufen sind eingeladen, sich an diesem zukunftsorientierten Modell zu beteiligen, in dem - allen modernen Erkenntnissen der Forschung entsprechend - eine Entscheidung über die weitere Bildungslaufbahn im Alter von 14 Jahren gezielt vorbereitet wird.

IV.4.20 Über die Bund-Länder-Initiative Erwachsenenbildung ist das kostenlose Nachholen von Bildungsabschlüssen möglich. Mit dem Gesetz zum Kurssystem in der Oberstufe wird das Wiederholen von Schulstufen eingeschränkt. Auch in der modularen Oberstufe sollen die individuelle Förderung sowie Frühwarnsysteme Schulabbruch vorbeugen helfen.

IV.4.21 Im Bereich der Berufsbildung werden zahlreiche Aktivitäten unternommen, um leistungsschwächeren SchülerInnen durch gezielte Förderangebote im ersten Jahr einer berufsbildenden mittleren oder höheren Schule zu unterstützen. Die LehrerInnen werden zunehmend für das Thema Drop-out und Schulabbruch sensibilisiert, dazu wird es ab Herbst eine neue Handreichung geben, in der entsprechende Forschungsergebnisse zur Professionalisierung von LehrerInnen einfließen.

IV.4.22 Für SchülerInnen mit einer anderen Erstsprache als Deutsch werden verschiedene Maßnahmen gesetzt: 
- Vorschulische Förderung: Sprachstandfeststellung und Deutschförderung im Kindergarten; Entwicklung und Implementierung des BESK-DaZ [Bogen zur Erfassung der Sprachkompetenz von Kindern mit Deutsch als Zweitsprache] (ab 2009/10)

- Schulische Förderung: Ausbau der Deutschförderung für Schülerlnnen mit anderen Erstsprachen als Deutsch an allgemeinbildenden und berufsbildenden Schulen (für 50.000 SchülerInnen)

- Ausweitung der „Sprachstandsförderkurse“ für außerordentliche SchülerInnen mit Deutsch als Zweitsprache )

- Ausbau des Erstsprachenunterrichts: 22 Sprachen, davon profitieren ca. 31.000 SchülerInnen

Ergänzend dazu gibt es laufende Maßnahmen in der Aus- und Fortbildung von PädagogInnen:

- Lehrerlnnenbildung: zunehmende Stärkung der Aspekte Mehrsprachigkeit, interkulturelle Kompetenz und Migrationspädagogik in der Aus- und Fortbildung an den Pädagogischen Hochschulen.

- Vielfalt in pädagogischen Berufen: Initiative zur interkulturellen Öffnung der Pädagogischen Hochschulen (PädagogInnen mit Migrationshintergrund, Deutsch als Zweitsprache, muttersprachlicher Unterricht, Diversität und Führung, Mehrsprachigkeit....)

IV.4.23 Weiters werden Maßnahmen in den Bereichen „Professionalisierung von LehrerInnen, SchulleiterInnen und der Schulaufsicht“, und „Unterstützung von Eltern mit Migrationshintergrund" gesetzt.

IV.4.24 Ein zentrales Instrument zur Verbesserung des Bildungsniveaus ist die Maßnahme PädagogInnenbildung NEU. Im Juni 2011 hat die Vorbereitungsgruppe zur PädagogInnenbildung NEU ihre Empfehlungen für die Struktur der Ausbildung der PädagogInnen vorgelegt, Eckpunkte der Curricula definiert und die Anforderungen bzw. den unmittelbaren Handlungsbedarf für die Trägerinstitutionen von PädagogInnenbildung dargestellt. Aus dem identifizierten Handlungsbedarf haben sich weitere Schritte im Reformprozess abgeleitet.

Im Oktober 2011 wurde der Schwerpunkt „Drei Reformpakete für die Pädagogischen Hochschulen“ gesetzt. Im Dezember 2011 wurde ein neues Dienstrecht für Lehrende an Pädagogischen Hochschulen vorgelegt, Doktorats-, und Habilitations- und Stipendienprogramme für die Pädagogischen Hochschulen im Bereich Pflichtschule, insbesondere Sekundarstufe I, eingerichtet und neue Studienprograme (Masterprogramme für MentorInnen, LerndesignerInnen und Schulmanagement) ausgeschrieben.

Am 29. Februar 2012 wurde zur Begleitung und Weiterentwicklung der Trägerinstitutionen von PädagogInnenbildung ein „Entwicklungsrat PädagogInnenbildung NEU“ eingerichtet.

IV.4.25 Die Nationale Strategie zum Lebenslangen Lernen wurde im Juli 2011 von der Bundesregierung beschlossen. Die Task Force bestehend aus Vertreterlnnen der 4 beteiligten Ministerien (BMUKK, BMWF, BMASK und BMWFJ) wurde konstituiert. Die Einrichtung der Nationalen Plattform erfolgt im Frühjahr 2012. Konkrete Umsetzungsergebnisse

- Die Neue Mittelschule

- Ausbau der schulischen Tagesbetreuung

- Oberstufe NEU

- $15 a$ B-VG Vereinbarung zum Nachholen von Bildungsabschlüssen

- Interministerielle Akkordierung der Eckpunkte und Grundlagen für die Etablierung des NQR 


\section{Neue Initiativen und geplante Maßnahmen}

IV.4.26 Seit der Übermittlung des letzten Beitrages zum NRP im Jahr 2011 wurden weitere neue Projekte ins Leben gerufen beziehungsweise entsprechend vertieft:

- Die neue Reife- und Diplomprüfung: diese stellt sicher, dass alle Schülerlnnen unabhängig vom Schulstandort - den besten Bildungsabschluss erhalten; ein einheitlicher Schulabschluss macht Maturazeugnisse national wie auch international vergleichbar. Die Ergebnisse der Schulversuche werden vom Bundesinstitut für Bildungsforschung (BIFI) und den universitären Vertragspartnern zusammengefasst und analysiert; die daraus gewonnenen Erkenntnisse fließen in künftige bildungspolitische Entscheidungen ein.

- Nachholen von Bildungsabschlüssen: von 2012 bis 2014 soll es 12.400 Menschen ermöglicht werden, kostenfrei im Rahmen der Initiative Erwachsenenbildung wieder ins Bildungssystem einzusteigen. Für diese Initiative für bildungsferne Personen werden 56,4 Mio. € investiert.

- Berufsorientierung und Bildungsberatung: Die Berufsorientierung an Schulen gab es schon bisher - neu ist, dass die Wirksamkeit von Information, Beratung und Orientierung für Bildung und Beruf in der 7. und 8. Schulstufe durch einen verbindlichen Maßnahmenkatalog verbessert werden soll.

- Jugendcoaching und Lehrlingscoaching: Diese neuen Maßnahmen bieten ab 2012 Beratung, Begleitung und Unterstützung für SchülerInnen bzw. Lehrlinge zur Dropout-Vermeidung (siehe Beschäftigungskapitel).

\section{Attraktivität, Qualität und Durchlässigkeit der beruflichen Bildung erhöhen}

IV.4.27 Ziel von QIBB (QualitätsInitiative BerufsBildung), ist die Entwicklung und dauerhafte Einführung eines umfassenden Qualitätsmanagementsystems im berufsbildenden Schulwesen zur Sicherung und Weiterentwicklung der Unterrichtsqualität und der Qualität der Verwaltungsleistungen auf den Steuerungsebenen Bund, Land, Schulen.

- Seit 2009: Implementierung Peer Review in QIBB - Evaluationsverfahren zur Qualitätsentwicklung am Schulstandort

- 2011-2013: Entwicklung von Kompetenzprofilen und eines Rahmencurriculums für Qualitätsverantwortliche in QIBB

- Gesetzliche Verankerung des Qualitätsmanagements als Aufgabe der Schulaufsicht bzw. des/der Schulleiter/in

IV.4.28 Lehre mit Matura: Seit Beginn des Schuljahres 2008/09 ist es möglich, parallel zur Lehre kostenfrei die Berufsreifeprüfung abzulegen. Seit April 2009 gibt es in allen Bundesländern Verträge mit Trägerorganisationen. Lehrgänge werden von Einrichtungen der allgemeinen und berufsorientierten Erwachsenenbildung (bfi, WIFI, VHS) und weiterführenden Schulen (Berufsschulen, höhere Schulen) angeboten. Waren es im Jahr 2008 noch 2.088 TeilnehmerInnen, so konnten im November 2011 bereits über 9.000 TeilnehmerInnen, verteilt über das ganze Bundesgebiet, verzeichnet werden.

\section{IV.5 Verminderung von Armut und sozialer Ausgrenzung}

IV.5.1 Österreich hat im Rahmen des fünften Kernziels der Strategie Europa 2020 die Bestrebung formuliert, die Anzahl der Personen in armuts- und ausgrenzungsgefährdeten Lagen innerhalb von zehn Jahren um mindestens 235.000 zu reduzieren. Die Zielgruppe wurde auf EU-Ebene mit den Indikatoren „Armutsgefährdung“, „erhebliche materielle Deprivation" und "Haushalte mit sehr niedriger Erwerbstätigkeit" erfasst. Aus der 
Indikatorenzusammensetzung ergibt sich hinsichtlich der Zielerreichung eine enge Verbindung zwischen diesem Europa 2020 Kernziel zur sozialen Eingliederung und jenen im Bereich Bildung wie auch mit den Zielsetzungen zur Beschäftigungssteigerung.

Meilensteinprojekte und neue Initiativen

- Pflegefonds: 1,335 Mrd. € 2011 - 2016

- Bedarfsorientierte Mindestsicherung

- Plattform Armutsbekämpfung

- Jugendcoaching

- Arbeitsmarktoffensive: 750 Mio. $€$

- Verbesserte Arbeitsmarkteinbindung von gesundheitlich beeinträchtigten Personen

- Bekämpfung der Kinderarmut: Kostenloses verpflichtendes Kindergartenjahr und Ausbau der Ganztagesbetreuung

\section{Neue Initiativen und geplante Maßnahmen}

IV.5.2 Mittels des durch das Pflegefondsgesetz im Juli 2011(siehe auch Beschäftigungskapitel und nationaler Sozialbericht) ${ }^{40}$ konstituierten Pflegefonds werden folgende Ziele verfolgt:

- Sicherung des bestehenden Angebots sowie Förderung des bedarfsgerechten Ausund Aufbaus des Angebots an Betreuungs- und Pflegedienstleistungen in der Langzeitpflege der Länder und Gemeinden;

- Weiterentwicklung der bedarfsgerechten Versorgung pflegebedürftiger Personen und ihrer Angehörigen mit bedürfnisorientierten und leistbaren Betreuungs- und Pflegedienstleistungen im Bereich der Langzeitpflege;

- Harmonisierung im Bereich Betreuungs- und Pflegedienstleistungen der Langzeitpflege;

- Verbesserung der Transparenz, Validität und Vergleichbarkeit von Pflegedienstleistungsdaten im Wege einer österreichweiten adäquaten Pflegedienstleistungsdatenbank und -statistik (ab Mitte 2012).

Der Pflegefonds wird Zweckzuschüsse von insgesamt 685 Mio. $€$ an die Länder zur teilweisen Abdeckung des Aufwands für die Sicherung sowie den bedarfsgerechten Aus- und Aufbau des Betreuungs- und Pflegedienstleistungsangebots in der Langzeitpflege für die Jahre 2011 bis 2014 gewähren. Im Zuge des Konsolidierungspaketes wurden nun für die Jahre 2015 und 2016 insgesamt weitere 650 Mio. € vereinbart, um diesen Weg fortzusetzen. Außerdem wird einer Strukturarbeitsgruppe entsprechende Vorschläge für weitere Reformen zur nachhaltigen Sicherung und Weiterentwicklung der Pflegedienste und die Fortführung des Pflegefonds unter Einbeziehung aller Stakeholder bis Ende 2012 erarbeiten.

IV.5.3 Die Einbeziehung der Akteure und Betroffenen bei der Ausarbeitung und Umsetzung von Armutsbekämpfungs- und Eingliederungsmaßnahmen ist ein zentrales Element. Um der Multidimensionalität der Armuts- und Ausgrenzungsbekämpfung Rechnung zu tragen und zur Begleitung der Umsetzung des Kernzieles in diesem Bereich sowie zur konkreten Ausgestaltung der korrespondierenden Leitinitiative wurde mit allen relevanten Akteurinnen in Österreich ein dauerhafter Dialog etabliert. Dieser Diskussionsprozess zur vertieften Flankierung des Europa 2020 Prozesses ist in Form von regelmäßigen Plattformtreffen

\footnotetext{
${ }^{40}$ Ab 2012 sollen die Mitgliedstaaten jährlich ergänzend zu den NRPs über die aktuellen Entwicklungen und Strategien im Bereich der OMK Sozialschutz und soziale Eingliederung berichten und vertiefende Informationen zu den Themenbereichen der OMK - soziale Eingliederung, Renten und Gesundheit/Langzeitpflege - vorlegen. Für Österreich wird dieser Bericht im Mai 2012 vorgelegt werden und einen Schwerpunkt im Bereich Langzeitpflege umfassen.
} 
konzipiert, die mindestens halbjährlich stattfinden und durch weitere Treffen zu spezifischen Themen in Unterarbeitsgruppen ergänzt werden.

\section{Strategische Ausrichtung und Umsetzung bestehender Maßnahmen}

IV.5.4 Armut und Ausgrenzungsgefährdung rückte in besonderem Maße seit Beginn der Wirtschafts- und Finanzkrise im Jahr 2008 in das Zentrum politischen Handelns in Österreich. Vor diesem Hintergrund ist es daher ein vorzeigbares Ergebnis, dass zwischen 2008 und 2010 eine Reduktion der Armuts- und Ausgrenzungsgefährdung in Österreich um nahezu 160.000 Personen erreicht werden konnte. Ermöglicht wurde dies u.a. mit politischen Maßnahmen zur Steigerung fairer Chancen für den Beschäftigungseinstieg Jugendlicher, mit Förderprogrammen im Bereich Bildung sowie mit anderen zielgruppenspezifischen Maßnahmen zur Förderung der Eingliederung erwerbsfähiger, arbeitsmarktferner Personen. Zusätzlich wurden die Bemühungen zur Verbesserung der Arbeitsplatzqualität verstärkt und finanzielle Anreize zur Beschäftigungsaufnahme bzw. zum Verbleib in Beschäftigung gesetzt. Die dadurch erreichte Verbesserung der Einkommenssituation der Haushalte wirkte sich in der Folge auch positiv auf die Bekämpfung der Kinderarmut aus (siehe Beschäftigungskapitel).

IV.5.5 Diese erfolgreiche Strategie, die umfassend das Prinzip der aktiven Eingliederung verfolgt, wird 2012 fortgesetzt, wobei insbesondere vor dem Hintergrund einer alternden Gesellschaft neben der sozialpolitischen Fokussierung auf die Zielgruppen Langzeitarbeitslose, WiedereinsteigerInnen, Jugendliche und Kinder nun noch verstärkt auf die arbeitsmarktpolitischen Bedürfnisse von Personen mit gesundheitlichen Beeinträchtigungen und Menschen mit Behinderung eingegangen wird.

IV.5.6 Ein weiterer Schwerpunkt, der 2012 fortgesetzt wird, besteht in der Prävention der Vererbung von Armut. Hier sollen an der Schnittstelle zwischen Bildung, Ausbildung und Beruf Betreuungsangebote für sozial benachteiligte Kinder- und Jugendliche ansetzen. Förderungen im Bildungsbereich betreffen zum einen den Ausbau des ganztägigen Schulangebots, zum anderen sollen zielgruppenspezifische Programme (z.B. für MigrantInnen) die Situation armutsgefährdeter Gruppen nachhaltig und generationenübergreifend verbessern (siehe auch Bildungskapitel).

\section{Bekämpfung der Langzeitarbeitslosigkeit durch eine Verbesserung der Erwerbsbeteiligung armuts- und ausgrenzungsgefährdeter Gruppen im erwerbsfähigen Alter}

IV.5.7 Im Bereich Bekämpfung der Langzeitarbeitslosigkeit setzt die österreichische Bundesregierung auf Maßnahmen zur Verbesserung der Erwerbsbeteiligung armuts- und ausgrenzungsgefährdeter Gruppen im erwerbsfähigen Alter (siehe auch Beschäftigungsziel). Einige der Maßnahmen werden im Rahmen des bestehenden operationellen Programms Beschäftigung des Europäischen Sozialfonds (ESF) umgesetzt. Einen guten Überblick über die Vielfalt der im Rahmen des ESF geförderten Projekte bietet die Website http://www.esf.at/esf/projekte/.

IV.5.8 Die Einführung der Bedarfsorientierten Mindestsicherung (BMS), wurde im Oktober 2011 bundesweit abgeschlossen. Sie harmonisiert die unterschiedlichen Sozialhilferegelungen der Bundesländer und ermöglicht eine gezielte Förderung der Erwerbsbeteiligung besonders gefährdeter Bevölkerungsgruppen. Durch die auf diesem Weg erreichte stärkere Einbeziehung der früheren SozialhilfebezieherInnen in das aktive arbeitsmarktpolitische Angebot des Arbeitsmarktservice (AMS) wird die soziale Eingliederung langzeitarbeitsloser Risikogruppen nachhaltig durch aktivierende Initiativen gefördert. 
IV.5.9 Vor allem auch für ältere Langzeitbeschäftigungslose werden in Gestalt von Lohnsubventionen und Eingliederungsbeihilfen verstärkt finanzielle Mittel verwendet; darüber hinaus soll eine umfassende Informationsoffensive die Bereitschaft von Unternehmen fördern, ältere ArbeitnehmerInnen zu beschäftigen.

IV.5.10 Qualifizierungsmaßnahmen und die laufende Verbesserung der Arbeitsqualität sind weitere Ansatzpunkte, die eine Aufnahme von bzw. einen Verbleib in Beschäftigung fördern sollen. Auch das langfristige Zurückdrängen diverser Formen prekärer Beschäftigung sowie die Bekämpfung von Einkommensarmut ermöglichen hier ergänzende Synergieeffekte.

\section{Maßnahmen zur Gesundheitsprävention am Arbeitsplatz und verstärkte Arbeitsmarkt(re)integration von Menschen mit gesundheitlichen Einschränkungen und Menschen mit Behinderungen}

IV.5.11 Einen weiteren Schwerpunkt bilden die Gesundheitsprävention am Arbeitsplatz und die verstärkte Arbeitsmarkt(re)integration von Menschen mit gesundheitlichen Einschränkungen und Menschen mit Behinderungen. Damit ältere oder gesundheitlich beeinträchtigte Menschen länger im Erwerbsleben bleiben können, wird ein umfassendes Angebot zum Erhalt der Gesundheit bzw. beruflichen Wiedereingliederung geschaffen. So hat z.B. die Wirtschaftskammer Österreich mit Sozialversicherungen, Sportvereinen, und sonstigen Anbietern im Gesundheitsbereich die Initiative „proFITNESS: Gesunde MitarbeiterInnen - Gesundes Unternehmen“ ins Leben gerufen, um KMUs bei der Ergreifung von Maßnahmen der betrieblichen Gesundheitsförderung zu unterstützen, ihnen einen Überblick über die bestehenden Angebote zu geben und sie von den Vorteilen betrieblicher Gesundheitsförderung zu überzeugen. Mit dem Stabilitätspaket wurde u.a. eine große Arbeitsmarktoffensive vereinbart, um älteren Arbeitnehmerlnnen oder gesundheitlich eingeschränkten Menschen neue Chancen zu eröffnen. Damit wird angestrebt, die Zahl der Frühpensionierungen zu verringern, ohne im gleichen Maße die Kosten für Arbeitslosigkeit zu erhöhen. Bei allen Maßnahmen wird auf die soziale Ausgewogenheit geachtet.

IV.5.12 Gesundheitlich beeinträchtigte Personen unter 50 Jahren werden ab 2014 in die berufliche Rehabilitation beim AMS einbezogen. Grundsätzlich soll die berufliche Rehabilitation Ausbildungen zu einer Berufstätigkeit umfassen, die dem bisherigen Qualifikationsniveau entsprechen.

IV.5.13 Bei Maßnahmen zur Integration von Menschen mit Behinderungen in das Erwerbsleben bilden die begleitenden Hilfen einen besonderen Schwerpunkt. In Form einer individuellen Beratung und Begleitung von Jugendlichen an der Schnittstelle Schule / Beruf leisten sie einen wichtigen Beitrag zur nachhaltigen Erstintegration benachteiligter junger Menschen in den offenen Arbeitsmarkt.

\section{Reduktion der frauenspezifischen Benachteiligungen in den Bereichen Einkommen und Erwerbsbeteiligung}

IV.5.14 Eine langfristige Herausforderung besteht in der Verbesserung der Einkommenschancen, der Vereinbarkeit von Beruf und Familie sowie in der Erhöhung der Erwerbsbeteiligung von Frauen und der frauenspezifischen Benachteiligung in den Bereichen Einkommen und Erwerbsbeteiligung gegenzusteuern. Die verstärkte Erwerbsbeteiligung von Frauen führte trotz Krise zu einer verbesserten Einkommenssituation der Haushalte und zu einer Reduktion von Kinderarmut (Maßnahmen siehe Beschäftigungskapitel).

Allerdings sind Haushalte mit Frauen als primäre Einkommensbezieherinnen überdurchschnittlich armutsgefährdet. Alleinerzieherinnen bilden eine besonders gefährdete Risikogruppe und sind daher prioritäres Ziel geschlechtsspezifischer Maßnahmen. 
IV.5.15 Die Bundesregierung setzt Maßnahmen, um die Entwicklung des Gender-Pay-Gaps bis 2020 in Richtung des EU-27-Durchschnitts zu bewegen. Zu diesem Zweck wurden zum einen Schritte gesetzt, die eine Erhöhung der Einkommenstransparenz zur Folge haben, zum anderen sollen Bewusstseinskampagnen bereits bei jungen Mädchen ansetzen und diese bei ihren Berufsentscheidungen positiv unterstützen (siehe Beschäftigungskapitel).

IV.5.16 Auch die laufende Verbesserung des Betreuungsangebots für Kinder und pflegebedürftige Angehörige beschleunigt diese Bemühungen, da Frauen einen überproportionalen Anteil familiärer Arbeit übernehmen. Die Förderung der Väterkarenz soll in Hinblick auf diese Benachteiligung eine Verhaltensänderung unterstützen.

\section{Bekämpfung der Kinder- und Jugendarmut und der Vererbung von Armut}

IV.5.17 Das Risiko negativer Auswirkungen von Armut bei Kindern und Jugendlichen auf die weiteren Chancen im Leben stellt ein erhebliches Problem dar. Einerseits können Synergieeffekte aller bisher vorgestellten Maßnahmen das Risiko familiärer Armut verringern, andererseits werden konkrete Maßnahmen in den Bereichen Bildung, Arbeitsmarkt und Integration gesetzt, um den Übergang an der Schnittstelle Schule / Ausbildung und Beruf möglichst erfolgreich zu gestalten. Spezielle zielgruppenspezifische Förderung soll die intergenerationelle Vererbung von Armut unterbinden. Aktive Maßnahmen zur Senkung der Schulabbrecherlnnenquote sind an dieser Stelle ebenso wichtig wie die frühzeitige sprachliche Förderung von Kindern mit Migrationshintergrund (siehe Kapitel Beschäftigung und Bildung).

IV.5.18 Um der Multikausalität von Armutsentstehung bei Jugendlichen zu begegnen wird ein umfassender Ansatz verfolgt. Neben Maßnahmen aus dem Bildungs- und Arbeitsmarktbereich (z.B. Ausbildungsgarantie), wurde das Angebot des Jugendcoaching geschaffen, um Jugendliche bei der Wahl ihrer Ausbildung und ihres Berufsweges zu unterstützen (siehe auch Euro-Plus-Pakt). Das breit angelegte Konzept unterstützt die persönliche und soziale Stabilisierung von Jugendlichen und bietet Perspektiven und Lösungskompetenz vor allem auch vor dem Hintergrund besonders schwieriger familiärer und sozialer Verhältnisse.

\section{Förderung der Vereinbarkeit von Familie und Beruf}

IV.5.19 Auch die Einführung eines kostenlosen verpflichtenden Kindergartenjahres verbessert die Bildungschancen von u.a. benachteiligten Kindern und schafft gleichzeitig bessere Voraussetzungen für die Erwerbsbeteiligung von Eltern und unterstützt die Vereinbarkeit von Familie und Beruf.

IV.5.20 Eine wesentliche Voraussetzung für die Verbessrung der Einkommenschancen und Erwerbsbeteiligung von Frauen ist eine bessere Vereinbarkeit von Familie und Beruf durch einen Ausbau der Kinderbetreuungs- und (u.a. ambulanten) Pflegebetreuungseinrichtungen. Dafür werden zusätzliche Mittel vom Bund und den Bundesländern zur Verfügung gestellt. Neben dem Ausbau der Kinderbetreuungsangebote für die Unter-6-Jährigen wird auch die Ganztagesbetreuung in Schulen ausgeweitet, und ermöglicht aufgrund der besseren Ausgangsposition für die Aufnahme einer Erwerbsarbeit eine Verbesserung der Einkommenssituation der Haushalte.

IV.5.21 Ein-Eltern-Haushalte sowie kinderreiche Familien, die zu den Risikogruppen in Bezug auf Armutsgefährdung zählen, werden vom Ausbau von Kinderbetreuungseinrichtungen besonders profitieren. 


\section{IV.6 Wettbewerb und unternehmerisches Umfeld}

IV.6.1 Die Schwerpunkte in diesem Bereich liegen auf der Verbesserung des Finanzierungszugangs heimischer KMU, auf der Stärkung der Wettbewerbsfähigkeit und der weiteren Internationalisierung. Viele der Maßnahmen, die bereits im Österreichischen Reformprogramm 2011 genannt wurden, waren auf mehrere Jahre angelegt und werden dementsprechend fortgeführt.

Meilensteinprojekte und neue Initiativen

- Reform des Wettbewerbs- und Kartellrechts

- KMU Fitness Paket

- Unternehmensserviceportal

- Umsetzung der Dienstleistungsrichtlinie

\section{Neue Initiativen und geplante Maßnahmen}

IV.6.2 Mit der Umsetzung der EU-Dienstleistungsrichtlinie wurden wesentliche rechtliche und administrative Hindernisse im Handel mit Dienstleistungen beseitigt und der länderspezifischen Empfehlung vom Juni 2011 entsprochen.

Länderspezifische Empfehlung Nr. 5: „..... die Annahme der
ausstehenden „horizontalen Rechtsvorschriften“ zur

Umsetzung der Dienstleistungsrichtlinie beschleunigen ..."
Die Dienstleistungsrichtlinie (DL-RL) 2006/123/EG regelt die Liberalisierung des (grenzüberschrei-tenden) DLVerkehrs (Niederlassung und Erbrin-gung von Dienstleistungen) und war bis 28.12.2009 in nationales Recht umzusetzen. Ein Bundes- und neun Landesgesetze regeln die jeweils in ihre Zuständigkeit fallenden horizontalen Elemente. Das Dienstleistungsgesetz auf Bundesebene enthält folgende Regelungen:

- Es verweist auf die auf Landesebene einzurichtenden Einheitlichen Ansprechpartner (9 EAP). In Verfahren erster Instanz können schriftliche Anbringen beim Einheitlichen Ansprechpartner eingebracht werden (Fristenlaufbeginn 3 Werktage nach Einbringung beim EAP). Der EAP leitet diese dann an die zuständige Stelle weiter.

- Es legt die Informationspflichten der EAP gegenüber den Dienstleistungserbringern und -empfängern sowie die Mitwirkungspflichten der Behörden fest.

- Anträge können elektronisch bei der Behörde eingebracht werden. Die Behörde muss auch elektronisch zustellen können.

- Das DLG bietet den Materiengesetzgebern die Möglichkeit, in ihren Gesetzen mittels Opt-In auf das DLG und die dort vorgesehene Genehmigungsfiktion im Verfahren zu verweisen (= Anträge gelten als von Gesetzes wegen erteilt, wenn der Bescheid nicht innerhalb der vorgesehenen Frist von der Behörde erlassen wird).

- Das DLG regelt die grenzüberschreitende Zusammenarbeit zwischen Verwaltungsbehörden (via das e-Informationssystem IMI) und legt Verbindungsstellen für die Bundesebene fest.

- Weiters sind im DLG Informationspflichten des Dienstleistungserbringers gegenüber dem Dienstleistungsempfänger vorgesehen.

Das DLG auf Bundesebene trat am 21. November 2011 in Kraft. Die entsprechenden DLG auf Landesebene enthalten ähnliche horizontale Elemente, die in die Zuständigkeit der Länder fallen. Alle Bundesländer haben ihre horizontalen DLG bereits beschlossen. Eine vollständige Umsetzung der DL-RL wurde auch bereits nach Brüssel gemeldet. 


\section{Wettbewerb und unternehmerisches Umfeld}

IV.6.3 Mit der Reform des Wettbewerbs- und Kartellrechts soll die Wettbewerbspolitik und Innovationskraft in Österreich gestärkt werden. Dazu wurden Gesetzesentwürfe ausgearbeitet, die bis Sommer im Parlament beschlossen werden sollen. Die Novellierung des Wettbewerbsgesetzes soll insbesondere die weisungsfrei gestellte Bundeswettbewerbsbehörde (BWB) stärken. Ihre Ermittlungsbefugnisse sollen an jene der EU-Kommission angeglichen und Auskunftsverlangen künftig schneller -

Länderspezifische Empfehlung Nr. 5: „........ die nämlich per Bescheid - durchgesetzt Befugnisse der Wettbewerbsbehörde stärken....." werden. Zudem wird die Kronzeugenregelung verbessert. Parallel dazu sorgt eine Novelle des Nahversorgungsgesetzes dafür, dass die Wettbewerbsbehörden einen eventuellen Preismissbrauch durch marktbeherrschende Versorger im Strom- und Gasbereich künftig leichter nachweisen können. Vorbild für diese Neuregelung, die insbesondere eine erhöhte Mitwirkungspflicht der Energieversorgungsunternehmen vorsieht, ist Deutschland. Dies gilt auch für die Bestimmungen über die kollektive Marktbeherrschung, die neu im österreichischen Recht aufgenommen werden soll. Im Kartellrecht soll die Aufsicht wirksamer und transparenter gestaltet werden, indem insbesondere Schlupflöcher geschlossen werden. Mehr Transparenz für Konsumenten und Unternehmen soll geschaffen werden.

IV.6.4 Im Hinblick auf die länderspezifische Empfehlung, ungerechtfertigte Beschränkungen von Gewerbe und Berufen zu beseitigen, ist anzumerken, dass in den letzten Jahren verschiedene Schritte zum Abbau von Beschränkungen gesetzt wurden: So sind z.B. Interdisziplinäre Gesellschaften zwischen

Länderspezifische Empfehlung Nr. 5: „.... Förderung des indem die Markteintrittschancen gelockert, ungerechtfertigte Beschränkungen von Gewerbe und Berufen beseitigt werden ....."
Wettbewerbs (...) insbesondere im Dienstleistungssektor,

Bilanzbuchhaltern und Steuerberatern bereits seit einigen Jahren möglich, zwischen Baumeistern und Ziviltechnikern sind sie angedacht. Nachdem der Europäische Gerichtshof im Mai vergangenen Jahres erkannt hatte, dass die Zugangsbeschränkungen zum Beruf des Notars gegen EURecht verstoßen, wurde die

Notariatsordnung im November 2011 entsprechend novelliert. Im Bereich des Gewerberechts wird laufend darauf geachtet, die Bedingungen für Wirtschaftstreibende zu verbessern: Neben diversen Richtlinienumsetzungen, etwa der Richtlinie 2008/48/EG über Verbraucherkreditverträge im Bereich der Kreditvermittler, wurde etwa ein neues Gewerbe "Wertpapiervermittler" geschaffen. Außerdem entfielen die bis dahin bestehenden betriebsanlagenrechtlichen Regelungen der Gewerbeordnung über Einkaufszentren. Weitere Novellierungen sind in Vorbereitung. Die Berufsgruppe der Personenbeförderung mit PKW (Taxigewerbe) ist von den Liberalisierungen durch das Gemeinschaftsrecht (noch) nicht betroffen, diese Gruppe wurde allerdings in Österreich hinsichtlich des Markt- und Berufszugangs weitgehend parallel zum harmonisierten Bereich der Busbeförderung gestaltet. 


\section{Förderung von Unternehmertum / Gründungdynamik}

IV.6.5 Im abgelaufenen Jahr lag der Fokus auf der Förderung von jungen Unternehmen. Die Umstrukturierung der Jungunternehmerförderung der Austria Wirtschaftsservice GmbH (aws) wurde in geänderten Richtlinien und Programmdokumenten, die mit 1. Jänner 2012 in Kraft getreten sind, umgesetzt.

IV.6.6 Das KMU Fitness Paket, welches im Mai 2011 initiiert wurde richtet sich an Unternehmensgründer sowie kleinere und mittlere Unternehmen als Zielgruppe. Das Paket in Form eines Scheckbuches soll die Erneuerung des Unternehmensbestandes und den Strukturwandel unterstützen und bietet einen Überblick über bestehende Förderangebote. Mit dem Unternehmensgründungsprogramm bietet das AMS arbeitslosen Personen, die sich beruflich selbständig machen wollen, Unterstützung bei der Unternehmensgründung. Diese umfasst eine begleitende Unternehmensberatung, Weiterbildungsangebote und eine Gründungsbeihilfe.

IV.6.7 Um der österreichischen Eigenkapitalfinanzierungslandschaft weitere Impulse zu geben und innovativen Unternehmen den Zugang zu Beteiligungs- und Risikokapital zu ermöglichen, wurden neue marktkonform konzipierte Maßnahmen geschaffen. Dazu zählen die VC-Initiative, der Cleantech-Fonds und der Mittelstandfonds.

IV.6.8 Im Rahmen der Tourismusstrategie des Bundes wurden mit den Partnern auf Länderebene die Schwerpunkte für 2012 vereinbart. Zentrale Elemente sind neben der tourismuspolitischen Abstimmung zwischen Bund und Ländern und der verstärkten Zusammenarbeit im Tourismusmarketing die Förderung als Innovations- und Investitionsanreiz. Dieses Maßnahmenbündel soll dazu beitragen, die Wettbewerbsfähigkeit der österreichischen Tourismusbetriebe zu stärken.

IV.6.9 Mit dem Unternehmensserviceportal setzt Österreich eine Priorität des Jahreswachstumsberichts 2012 um. Das (USP, siehe www.usp.gv.at) ist bisher die größte einzelne Maßnahme im Rahmen der Initiative „Verwaltungskosten senken für Unternehmen“. Ziel ist es, das Portal als die elektronische Schnittstelle der Unternehmen zur öffentlichen Verwaltung zu positionieren. Das USP umfasst einen Informations- sowie einen Transaktionsbereich und soll es den Unternehmen möglichst einfach machen, ihre gesetzlichen Informationsverpflichtungen zu erfüllen. Das Informationsangebot wird nach relevanten Unternehmenssituationen wie Gründung, Steuern \& Finanzen, Umwelt \& Verkehr etc. aufgebaut. Die Umsetzung des USP erfolgt kontinuierlich. Das USP setzt in der ersten Ausbaustufe des Vollbetriebs auf zentrale Informationsbereitstellung und Verfahrenseinbindung mittels Single-Sign-On. In der zweiten Phase liegt der Schwerpunkt auf Prozessunterstützung für Unternehmen sowie die Vereinheitlichung und Harmonisierung von Meldepflichten. Dies soll dazu beitragen, den Kosten- und Zeitaufwand für Unternehmen zu senken und die Attraktivität des Wirtschaftsstandortes Österreich zu erhöhen und einen wesentlichen Beitrag zur Verwaltungsreform zu leisten. Im Laufe des Jahres 2011 wurde das USP sukzessive weiterentwickelt und um wesentliche Inhalte angereichert. Derzeit läuft ein Pilotbetrieb mit einigen ausgewählten Unternehmen, die im angemeldeten Bereich verschiedene Funktionalitäten testen. Der Pilotbetrieb dient zur Vorbereitung auf den Vollbetrieb des USP. Viele Behördenwege werden von Unternehmen dank des USP künftig deutlich einfacher und rascher als bisher erledigt werden können. Der Pilotbetrieb soll 2012 in einen Vollbetrieb münden.

Die Vorteile des Unternehmensserviceportals im Überblick:

- Einmal anmelden und verschiedene Anwendungen der Verwaltung nutzen (SingleSign-On)

- Informationen rund um die Uhr

- umfassende, auf die Unternehmen zugeschnittene Informationen 
- Kostenersparnis durch Online-Services bei Antragstellungen

- BenutzerInnen und deren Rechte in Verfahren zentral verwalten

einfache und einheitliche Zugangsvoraussetzungen

höchste Sicherheit

In den Erläuterungen zum USP Gesetz werden kurz- bis mittelfristige Entlastungen der Unternehmen in der Höhe von 100 Mio. € bzw. der langfristigen Entlastung um 300 Mio. € genannt.

IV.6.10 Mit der Initiative Verwaltungskosten senken für Unternehmen zur Unterstützung der Wirtschaft und Stärkung des Standortes setzt die Bundesregierung seit 2006 ein ambitioniertes Entbürokratisierungsprogramm für Österreichs Unternehmen um. Bis 2012 sollen Österreichs Unternehmen um mehr als $1 \mathrm{Mrd}$. € entlastet werden, 2010 konnte das erste Zwischenziel in Höhe von rd. 564 Mio. € erreicht werden.

Um das Gesamtziel zu erreichen sind nun alle für 2012 geplanten Maßnahmen konsequent umzusetzen. Die wichtigsten Maßnahmen sind

- e-Rechnung - die Gleichstellung der elektronischen Rechnung mit der Papierrechnung

- Unternehmensserviceportal Phase 2: Prozessoptimierung, Vermeidung Mehrfachmeldungen; Umsetzung 2012-2014

- Vereinfachungsmaßnahmen im Gewerberecht, Einrichtung Gewerberegister NEU, legistische Vereinfachungen, zentrale Infoplattform 


\section{Beiträge der Sozialpartner, Länder und Gemeinden}

Im österreichischen Reformprogramm 2011 wurden die Maßnahmen, die seitens der Sozialpartner, der Länder, Städte und Gemeinden zur Umsetzung und Erreichung der nationalen Europa 2020 Ziele dargestellt. Das vorliegende Reformprogramm 2012 knüpft am Vorjahresprogramm an und stellt „best practice“ Beispiele vor.

\section{Aktivitäten der österreichischen Sozialpartner zu Europa 2020 http://www.sozialpartner.at}

\section{Geplante Maßnahmen 2012}

- Bildungspolitischer Dialog 25.01.2012 mit den Ministerien für Wirtschaft, Soziales, Bildung und Wissenschaft: Duale Ausbildung fit für die Zukunft machen

- Enquete Integration Migration mit Präsentation der Studie der Sozialpartner - erstes Halbjahr

- Enquete Finanzmärkte und Rohstoffe - zweites Halbjahr

- Gemeinsames Positionspapier zur „Investitionen und Innovationsfähigkeit für künftiges Wachstum"

- Positionspapier „Europäische Wirtschaftspolitik zum Abbau der Ungleichgewichte“

- Bad Ischler Dialog 15.-16.102012 Thema „Zukunft Europa“

- Erarbeitung einer Toolbox mit praxisgerechten Leitfäden für Personalverantwortliche und Betriebsräte, speziell für einzelne Branchen, mit Tipps zu Themen rund um das richtige Altersmanagement in Betrieben; weiters ist eine Sozialpartner-Veranstaltung im Mai 2012 zu „Alter und Arbeit“ geplant

\section{Durchgeführte Maßnahmen 2011}

- Bildungsgipfel 2.2.2011 mit den Ministerinnen für Bildung und Wissenschaft: Gemeinsame Unterzeichnung einer Punktation zur künftigen Bildungsstrategie

- Bildungspolitischer Dialog 28.6.2011: Schwerpunktthema Hochschulbildung

- Enquete Finanzmarktregulierung 10.5.2011

- Sozialpartnereinigung zum Thema Mehrweggetränkeverpackungen plus Brief an zuständige Regierungsmitglieder

- Sozialpartnereinigung zum Thema Schließung der Kyoto-Lücke plus Brief an zuständige Regierungsmitglieder

- Bad Ischler Dialog der Sozialpartner im Oktober 2011 „Auswirkungen der Demographie auf Arbeitsmarkt und Sozialsysteme“, inkl. gemeinsames Positionspapier der Sozialpartner 
- Gemeinsames Positionspapier zur „Investitionen und Innovationsfähigkeit für künftiges Wachstum" - wird 2012 fertiggestellt

- Positionspapier „Europäische Wirtschaftspolitik zum Abbau der Ungleichgewichte“ wird 2012 fertiggestellt

- Europäisches Jahr des aktiven Alterns 2012: gemeinsame Vorbereitung der Maßnahmen und Initiativen mit dem BMASK; Veranstaltung der Sozialpartner zu Alter und Arbeit im ersten Halbjahr 2011

Gemeinsame Internetplattform der Sozialpartner für Bewusstseinsbildung und Austausch guter Praktiken

- www.arbeitundgesundheit.at: Plattform für betriebliche Gesundheitsförderung und Arbeitnehmerlnnenschutz

- www.arbeitundalter.at: Plattform für altersgerechte Arbeitsorganisation

- www.arbeitundbehinderung.at: Plattform für Integration von Menschen mit Behinderungen in den Arbeitsmarkt 


\section{Land Salzburg}

Im Land Salzburg wurde federführend durch die Landesamtsdirektion in Hinblick auf die Bedeutung der inhaltlichen Implementierung der Europa 2020 Strategie auf regionaler und kommunaler Ebene eine Europa 2020 Steuerungsgruppe Salzburger Bürgermeister initiiert.

Die Struktur, in der diskutiert wird, ist einzigartig. Jeder Bezirk des Landes Salzburg entsandte auf Basis eines Beschlusses in seiner jeweiligen Bürgermeister- bzw. Regionalverbandskonferenz maximal drei Bürgermeister in die Steuerungsgruppe. Mit der Nominierung von Stellvertretern für die Mitglieder wurde die Wichtigkeit dieser Einrichtung für die Bürgermeister noch zusätzlich betont.

Ziel der aktiven Steuerungsgruppe der Salzburger Bürgermeister ist es, EU-Fördermittel in der kommenden Strukturfondsperiode 2014 bis 2020 aufgrund der prozesshaften thematischen Befassung mit den Inhalten der Europa 2020 Strategie optimal zu nutzen und in den Regionen des Landes Salzburg einzusetzen. Der Schwerpunkt soll dabei insbesondere auf den Bereich des Klimaschutz-Kernziels in den Förderprogrammen gelegt werden.

Die Doppelnutzung von Wasseranlagen zur Stromerzeugung ist eines der Beispiele gemeinsamer bezirksübergreifender Projekte, die von der Europa 2020 Steuerungsgruppe der Salzburger Bürgermeister einwickelt wurde und zielgerichtet verfolgt wird.

Der Diskussionsprozess der 15 Salzburger Bürgermeister zur Implementierung der Europa 2020 Strategie gestaltet sich von Beginn an sehr dynamisch und unterstreicht die ergebnisorientierte, überkommunale Zusammenarbeit in der Steuerungsgruppe.

\section{Klimaschutz}

Aus den fachlichen und rechtlichen Rahmenbedingungen wird für das Land Salzburg folgender Zielpfad unter den Klimaschutzzielen bis zum Jahr 2050 abgeleitet und verfolgt:

- klimaneutral

- energieautonom

- nachhaltig

Als Konkretisierung der Zwischenziele (Detailziel) wurden folgende Punkte definiert: 2020

- Minus 30\% Treibhausgase (THG)

- $50 \%$ erneuerbare Energien

- Bilanziell hundertprozentige Versorgung der Landesgebäude durch Fernwärme und / oder erneuerbare Energieträger

2030

- Minus 50\% THG

- $65 \%$ erneuerbare Energien

- Bilanziell $100 \%$ Anteil erneuerbarer Energien für die Stromerzeugung in Salzburg sowie $100 \%$ solare Warmwasserbereitung

2040

- Minus 75\% THG

- $80 \%$ erneuerbare

- Bilanziell 100\% erneuerbare Energien in Einzelfeuerungen für Raumwärme

Die Zielsetzungen sind bilanziell pro Jahr zu verstehen, das Bezugsjahr ist jeweils 2005 (entspricht dem Bezugsjahr der EU 2020 Vorgaben). Die Treibhausgase werden als CO2Äquivalente unter Zugrundlegung des sogenannten Kyoto-6-basket ausgedrückt. 


\section{Frauenförderung / Antidiskriminierung}

Ausgehend von der Forderung der EU-Kommissarin Viviane Reding nach einer Frauenquote für die Wirtschaft, hat die Salzburger Landesregierung am 16. Mai 2011 beschlossen, den Frauenanteil in Aufsichtsräten von Unternehmungen, an denen das Land Salzburg beteiligt ist, bzw. bei der Besetzung von Organen von Unternehmen, für die das Land ein Entsendungsrecht hat, bis Ende 2014 auf 25 Prozent und bis Ende 2018 auf 35 Prozent zu erhöhen. Der Regierungsbeschluss, aus dem nun längerfristige Maßnahmen hervorgehen, befindet sich in der Umsetzungsphase.

\section{Land Kärnten}

\section{Forschung und Entwicklung}

Die KWF-Technologietransfer-Initiative verfolgt das Ziel, die Zusammenarbeit von Unternehmen mit Universitäten und Forschungsinstitutionen unter Einbindung der geeigneten Förderstellen zu stärken und auszubauen. Mit der gesamtheitlichen Erfassung, Gliederung und Aufbereitung der Forschungsaktivitäten wird für das Bundesland erstmalig eine solche Systematik erstellt.

Der Nutzen für Kärntens Unternehmen: Beschleunigung des Innovationsprozesses durch intensive Wissenskoppelung zwischen Wissenschaft und Wirtschaft, Aufbau von Grundlagenwissen, Zugang zu bestehenden und neuen Problemlösungskapazitäten sowie der Aufbau strategischer Allianzen mit Universitäten und Forschungseinrichtungen.

Es soll durch diese Maßnahme eine Erhöhung der innovations- und technologieaffinen Unternehmen von 270 bis 400 (bis 2013) erreicht werden.

\section{$\underline{\text { Innovation }}$}

Das Pilotprojekt „Kundenzentrierte Innovationen“ mit 15 teilnehmenden Unternehmen wurde mit der Zielsetzung gestartet, jene Unternehmen, die heuten och nicht oder nur sporadisch innovieren, auf ihrem Weg hin zur systematischen Innovationstätigkeit zu unterstützen. Anstöße für Innovationen kommen häufig von Kundenseite, um dieses Potenzial für die Entwicklung von neuen Produkten bzw. neuen Verfahren auszuschöpfen, stehen kleine und mittlere Unternehmen vor vielfältigen Herausforderungen. In Zusammenarbeit mit den teilnehmenden Unternehmen wurden in den einzelnen Prozessstufen Mindestanforderungen, Standardanforderungen sowie Best-in-Class-Lösungen erhoben.

\section{Land Steiermark}

\section{Wirtschaftsstrategie 2020 (WIST 2020)}

Die WIST 2020 wurde bereits in Ausrichtung auf die Europa 2020 Strategie erstellt und baut im Wesentlichen auf drei Leitthemen (Mobility, Eco-Tech, Health-Tech) und fünf Kernstrategien auf. Mit den Kernstrategien 2 - Innovations- und F\&E-Förderung, 3 Unternehmertum und Wachstum junger Unternehmen sowie 5 - Internationalisierung und Unternehmen am Standort, geht sie sowohl mit den einzelnen strategischen Zielsetzungen im NRP als auch mit den Leitlinien 4 und 6 der Europa 2020 Strategie konform und trägt damit auch zu deren Umsetzung bei. Damit verlinkt sie sich auch automatisch mit den Ideen und Ausrichtungen der künftigen Kohäsionspolitik ab 2014+. Die WIST 2020 wird auch zu Evaluierungszwecken mit dem EPIS (Evidenzbasiertes Policy Informationssystem) verknüpft. 


\section{Lehre mit Matura}

In der Steiermark wird das Modell Lehre mit Matura angeboten, welches vom Bundesministerium für Unterricht und Kunst unterstützt und gefördert wird. Ziel der Lehre mit Matura ist es, jungen Menschen welche sich für einen Lehrberuf entscheiden, die Möglichkeit zu geben zusätzlich zum Lehrabschluss auch einen Abschluss mit Matura zu ermöglichen.

Seit 2009 wird daher die Attraktivität eine Lehre zu absolvieren noch interessanter, da für Lehrlinge welche sich für dieses Modell entscheiden, eine höhere Ausbildung möglich ist. Dies ist ein wesentlicher Schritt, insofern sich für das weitere Berufsleben erheblich mehr Karrierechancen ergeben können.

\section{Land Tirol}

\section{Wirtschaftsleitbild Tirol 2011}

Mit dem vom Tiroler Landtag im Oktober 2011 beschlossenen Wirtschaftsleitbild Tirol 2011 werden wichtige strategische Handlungsfelder für die Wirtschaftspolitik Tirols definiert.

Die Ziele, Strategien, Maßnahmen umfassen bzw. zielen ab auf:

- Steigerung der Erwerbsquote

- Erhöhung der Arbeitsproduktivität

- Sicherung der internationalen Wettbewerbsfähigkeit

- Stärkung von Forschung und Entwicklung im privaten und öffentlichen Bereich

- Begrenzung der Ungleichheit der Einkommensverteilung

- Schaffung von gleichwertigen Lebensbedingungen im gesamten Land

- Absicherung außermarktlicher Dienste und Güter

Die Inanspruchnahme von Strukturfondsmittel erfolgt nach Maßgabe der gegebenen Möglichkeiten.

Eine Evaluierung der Umsetzungsmaßnahmen des Wirtschaftsleitbildes Tirol 2011 ist vorgesehen.

\section{Beschäftigungspakt Tirol}

Der Beschäftigungspakt Tirol versteht sich als regionale Vernetzung der Akteure der Tiroler Beschäftigungspolitik.

Als Strategie verfolgt der Beschäftigungspakt Tirol die koordinierte, strukturierte und konstruktive Zusammenarbeit aller Paktpartnerorganisationen in Angelegenheiten der Tiroler Arbeitsmarkt- und Beschäftigungspolitik.

Der Beschäftigungspakt Tirol ist strategisch auf die Maßnahmenbündelung und Umsetzung gemeinsamer Maßnahmen ausgerichtet, um auf Veränderungen am Arbeitsmarkt flexibel zu reagieren und Beiträge zu leisten, die

- ArbeitnehmerInnen zu unterstützen den Wandel in der Wirtschafts- und Arbeitsmarktstruktur zu bewältigen

- Arbeitslosigkeit bei besonders davon betroffenen Zielgruppen zu bekämpfen und zu verhindern

- die Ausgrenzung benachteiligter Personen am Arbeitsmarkt zu verhindern bzw. ihnen den Zugang zum Arbeitsmarkt erleichtern

- Gleichstellung von Frauen und Männern am Arbeitsmarkt zu fördern

- den Wissensaustausch von arbeitsmarkt- und beschäftigungspolitischen EntscheidungsträgerInnen verstärken 
Die Arbeitsschwerpunkte 2012-2013 des Beschäftigungspakts Tirol umfassen:

- Bildungs- und Berufsberatung

- Übergang Schule - Beruf

- Umsetzung der bedarfsorientierten Mindestsicherung

- Active Ageing

Die Inanspruchnahme von EFS-Mittel erfolgt nach Maßgabe der gegebenen Möglichkeiten.

Eine Evaluierung der Umsetzungsmaßnahmen des Beschäftigungspakts Tirol erfolgt laufend.

\section{Land Vorarlberg}

\section{Förderung niederschwelliger Beschäftigungsprojekte}

Das Land Vorarlberg und das AMS unterstützen gemeinsam Beschäftigungsprojekte, die arbeitsmarktpolitischen Problemgruppen eine möglichst rasche Reintegration in den Arbeitsmarkt bieten sollen. Besonders hervorzuheben sind vom Land finanzierte niederschwellige Beschäftigungsinitiativen, die vor allem auf Jugendliche bzw. jugendliche MigrantInnen abzielen.

Junge Menschen können beim Einstieg in das Erwerbsleben mit Hürden konfrontiert sein, wie eine abgebrochene schulische Ausbildung, problematische Familienstrukturen, Armut oder Migrationshintergrund. Die daraus resultierenden Benachteiligungen können mit Hilfe von arbeitsmarktrelevanten Maßnahmen bearbeitet und in begrenzter Zeit bewältigt werden. Ein Teil der Jugendlichen findet dadurch den Zugang zum Arbeitsmarkt. Eine - größenmäßig relevante - Untergruppe kann allerdings den Zugang zu diesen spezifischen Hilfestrukturen nicht bewältigen,

- weil sie die erforderliche Leistungsfähigkeit und Belastbarkeit nicht mitbringt

- weil sie aufgrund des spezifischen Verhaltens nur schwer in Ausbildung oder Erwerbsarbeit integriert werden kann.

Für diese Jugendliche werden die niederschwelligen Beschäftigungsprojekte angeboten. Die Jugendlichen kommen in diese Projekte freiwillig bzw. werden auf öffentlichen Plätzen auf das Beschäftigungsprojekt angesprochen. Es erfolgt keine Zuweisung durch das AMS. Die Jugendlichen werden aber angehalten, sich beim AMS als Arbeit suchend zu melden.

In den Projekten Werkstadt Bregenz, Job Ahoi!-Boote und Job-Ahoi!-Design sowie Startbahn Feldkirch und Bludenz bekommen die Jugendlichen die Möglichkeit einer stundenweisen Beschäftigung auf Basis des Tagelöhnerprinzips. Neben dem Angebot einer sozialpädagogischen Betreuung kann auch der Hauptschulabschluss nachgeholt werden. Ergänzend werden in den einzelnen Projekten Sprachkompetenztrainings angeboten werden.

Die Offene Jugendarbeit Dornbirn hat für ihre Projekte Job Ahoi!-Boote und Job-Ahoi!Design im Jahr 2011 den österreichischen „ESF Award“ erhalten.

Zur Messung des Erfolgs der Maßnahme wurde ein umfassendes Kennzahlensystem entwickelt, mittels dessen die Träger dem Land Vorarlberg die relevanten Informationen liefern. Dadurch kann auch frühzeitig steuernd in den Prozess eingegriffen werden. Die Statistiken des Arbeitsmarktes belegen die Erfolge der Maßnahmen. 


\section{$\underline{\text { Strategie Energiezukunft Vorarlberg }}$}

Im Rahmen des Programms Energiezukunft Vorarlberg wurde ein Landtagsbeschluss gefasst, wonach Vorarlberg bis 2050 energieautonom werden soll. Im November 2011 wurde von der Landesregierung eine Strategie bis 2020 verabschiedet. Diese enthält 101 konkrete Maßnahmen und detaillierte Zielsetzungen in allen Segmenten, vom Ausbau erneuerbarer Energieträger, über Gebäude, Mobilität und Raumplanung, Industrie bis zu Querschnittsmaßnahmen wie eine Bildungsoffensive. In einem Beteiligungsprozess wird nun darauf aufbauend die Umsetzung vorangetrieben.

Das Programm ist mit erheblichen Investitionen verbunden. Allein im Bereich Ausbau der erneuerbaren Energieträger ist mit Investitionen von etwas über 1 Mrd. e zu rechnen .Deutlich höher werden Investitionen für die thermische Sanierung des Gebäudebestandes liegen. Angestrebt wird hier eine Sanierungsrate von 3\%. Gerade in diesem Segment ist die Wertschöpfungs- und Beschäftigungswirkung besonders hoch.

Für die Umsetzung der Maßnahmen wird ein umfangreiches Monitoringsystem aufgebaut. In Bezug auf den Ausbau erneuerbarer Energieträger wird der Erfolg an der Zahl der jeweils, in den einzelnen Segmenten, realisierten Anlagen gemessen. Bei der thermischen Gebäudesanierung an der Zahl und Qualität der sanierten Gebäude.

\section{Land Wien}

Wiener Bedarfsorientierte Mindestsicherung

Mit 1. September 2010 ist das neue Wiener Mindestsicherungsgesetz (WMG) in Kraft getreten. Die Ziele wurden größtenteils erreicht:

a) Von der Erhöhung profitieren alle BezieherInnengruppen. Insbesondere die hohen Mindeststandards für Kinder haben für Familien mit Kindern eine deutliche Verbesserung zur Folge. Dies spiegelt wich auch in einem höheren Anteil der Kinder in der Bedarfsorientierten Mindestsicherung wider.

b) Die höheren Mindeststandards und vereinfachte Zugang zur Leistung (One-StopShop) haben zu einem Anstieg der monatlichen Fallzahlen geführt (ca. 17\% seit Einführung der Mindestsicherung). Die Folge ist eine Ausweitung des Anspruchskreises bzw. eine verringerte Non-Take-Up-Rate.

c) Die enge Verschränkung mit dem ersten sozialen Sicherungsnetz zeigt ebenfalls Wirkung. In Wien sind nunmehr alle MindestbezieherInnen krankenversichert und haben Zugang zu allen medizinischen Leistungen. Auch die Maßnahmen der Arbeitsintegration (Step2Job) greifen. Im Pilotprojekt konnten - trotzt oft sehr schwerer Rahmenbedingungen (Arbeitsmarkt und Zielgruppe) - rund 30\% der TeilnehmerInnen in den Arbeitsmarkt vermittelt werden.

d) Verbesserungsbedarf besteht hinsichtlich der Ausgestaltung des One-Stop-Shops. Dieser hat zu einer Aufblähung des Verwaltungsapparates geführt, da viele der gestellten Anträge mangels Erfüllung der Anspruchsvoraussetzungen abgelehnt werden mussten.

2012 soll die Bedarfsorientierte Mindestsicherung sowie die arbeitsmarktpolitischen Begleitmaßnahmen evaluiert werden. In Wien erfolgt hinsichtlich der Maßnahme Step2Job eine laufende Begleitevaluierung, die auch 2012 fortgesetzt wird.

Wiener Strategie für Forschung, Technologie und Innovation (Wiener FTI-Strategie)

Strategisches Ziel der FTI-Strategie ist, die Position Wiens als bedeutenden zentraleuropäischen Forschungs- und Innovationsstandort zu festigen und auszubauen. In 
fünf Handlungsfeldern werden Antworten auf die zentralen Herausforderungen für den FTIStandort Wien mit Perspektive bis zum Jahr 2015 gegeben.

2011 wurden unter anderen folgende Umsetzungsschritte gesetzt:

Die Initiative „WienWin“ sammelt Innovationen aus Wien und präsentiert sie VertreterInnen des Magistrats sowie der Unternehmungen der Stadt Wien, um innovative Vergaben zu fördern.

Der WWTF fördert „Vienna Research Groups for Young Investigators“ (Schwerpunkt Informations- und Kommunikationstechnologien) ebenso wie Call-Projekte im Rahmen des "Wiener Impulsprogramms für Geistes-, Sozial- und Kulturwissenschaften".

Der wichtigste Bezug zur nationalen Ebene ergibt sich aus dem vom Bund verfolgten Ziel einer Steigerung der Forschungsquote (Anteil am BIP) auf 3,76\% bis 2020. Nähere Informationen unter http://www.wiendenktzukunft.at/de.home.html.

\section{Stadt Bregenz}

\section{Environmental electronic democracy (e2d)}

Partizipation, Klimaschutz und eine faire Zukunft interessieren nach wie vor nur wenige Teile unserer Gesellschaft. Am Beteiligungs-Projekt nimmt nur noch ein geringer Anteil der ursprünglich gemeldeten Bürger teil. Auf Grund der kleinen Stichprobe sind Aussagen über den Effekt unterschiedlicher Beteiligungsmethoden mit Vorsicht zu genießen. In der OfflineGruppe, die persönlich und telefonisch betreut und informiert wird, sind weniger Teilnehmer ausgestiegen als in der Online-gruppe.

Sehr gut kommt die Besichtigung von regionalen Betrieben, welche sich aktiv für Klimaschutz und faire Bedingungen einsetzen, an. Die Teilnehmerzahl steigt bei jeder Veranstaltung kontinuierlich an. Das Feedback der Teilnehmer ist durchwegs positiv.

Die Aquise von Bregenzer Schulen zur Teilnahme an „KlimaDialogBregenz - e2d“ gestaltet sich schwierig - entweder überhaupt keine Rückmeldung oder Absage mangels freier Kapazitäten. Positiv ist zu vermerken, dass eine im Rahmen des Projekts unterstützte Multivisionsveranstaltung zum Thema „Fair Future“ von zahlreichen Bregenzer Schulen besucht wurde und gefühlsmäßig einem Großteil der Besucher die Themen Zukunftsfähigkeit, ökologischer Fußabdruck und Klimaschutz nachhaltig näher gebracht werden konnten.

Das zwar noch geringe, aber langsam steigende Interesse der Bevölkerung für Umweltengagement konnte durch die Besichtigung engagierter Unternehmen und Betriebe angereichert und unterstützt werden.

Sowohl die e2d-Zwischenveranstaltung als auch die Filmvorführung zum Thema haben gezeigt, dass junge Leute für das Thema sensibilisiert werden können.

Das e2d-Projekt läuft mit Sommer 2012 aus. Die im Bregenzer KlimaDialog durchgeführten Betriebsbesichtigungen für Bürger und Weiterbildungsangebote für den Schulbereich werden auch zukünftig - sofern das Interesse aufrecht bleibt - durchgeführt werden.

„Kind sein - Familie leben“

Das Projekt „Kind sein - Familie leben“ in Bregenz, mit dem Ziel der Erhöhung von Bildungschancen benachteiligter Kinder und Minderung sozialer Exklusion in Kindergärten und Schulen, hat bereits einiges in der Bildungslandschaft von Bregenz verändert. Vor allem das vernetzte Arbeiten aller Projektbeteiligten Partner ist ein wesentlicher Aspekt in der Umsetzung. Die Abstimmung von angestrebten Maßnahmen in den unterschiedlichen 
Einrichtungen und die gemeinsame Entwicklung von Richtzielen ist ein wichtiger Bestandteil dieser Netzwerkarbeit. Derzeit findet eine Finalisierung von Konzeptarbeit in allen Schwerpunktbereichen statt. Start der Umsetzungsmaßnahmen im Februar 2012. Neben den übergreifenden Konzepten zur Elternarbeit, Sprachförderung und Bildungszielen (Werte, Sozialkompetenz) wurden in den einzelnen Einrichtungen strukturelle Maßnahmen gesetzt: VS Schendlingen als Ganztagesschule, Umwandlung von Kindergärten in Familienzentren.

Besondere Umsetzungserfolge

- Ganztagesschule VS Schendlingen

- Kindergarten als Familienzentrum im KG an der Ach

- Sprachförderung als Querschnittthema im Kindergarten und durchgängige Qualifizierung der PädagogInnen

- Willenserklärung von Schule und Kindergärten gemeinsame Werte für die Bildungsarbeit in Bregenz zu entwickeln

Weiteres Vorgehen

- Antrag an den Fonds gesundes Österreich um die finanziellen Ressourcen zu erhöhen

- Weitere Durchführung der Teilprojekte

\section{$\underline{\text { WerkStadt Bregenz }}$}

Die Integra.gem. GmbH hat im Auftrag der Stadt Bregenz, des Landes Vorarlberg und den Gemeinden der Regionalplanungsgemeinschaft Bodensee das Jugendbeschäftigungsprojekt Bregenz umgesetzt. Gefördert wird das Projekt auch durch das Interreg IV Programm. Bis zu 16 Jugendliche finden in diesem Projekt eine Beschäftigungsmöglichkeit. Sie werden nach dem Tagelöhnerprinzip am Ende der Woche mit $5 €$ pro Stunde entlohnt, dürfen aber den Grenzwert der Geringfügigkeit nicht überschreiten. Mit der Gartenwerkstatt ist ein weiterer Tätigkeitsbereich dazu gekommen. Mit Aufträgen können Privatpersonen, Vereine und Firmen helfen: handwerkliche Arbeiten und Dienstleistungen wie Transporte, Maler-, Gartenund andere handwerkliche Allroundarbeiten werden gerne übernommen. Für die Stadt Bregenz reinigen und reparieren die Jugendlichen, die täglich mit E-Bikes und Anhänger unterwegs sind, 8 öffentliche Spielplätze. Arbeitsuchende Jugendliche, die mitarbeiten wollen, können sich jederzeit in der WerkStadt melden.

Jugendliche arbeiten im Schnitt drei Monate in der WerkStadt mit. In dieser Zeit werden sie in unterschiedlichste Arbeiten eingeführt und sie gewöhnen sich an regelmäßige Arbeitszeiten und werden an das Berufsleben heran geführt. Daneben werden sie von einer Sozialarbeiterln betreut, die sie bei der Jobsuche unterstützt. Viele Jugendliche finden nach drei, vier Monaten eine Lehrstelle, einen Job und können in den ersten Arbeitsmarkt integriert werden.

Die WerkStadt Bregenz ist stets voll belegt. Es sind immer Jugendliche auf der Warteliste.

Die WerkStadt Bregenz wird in dieser Form weitergeführt. Es werden stets neue Aufgabenbereiche erschlossen, damit es den Jugendlichen nicht langweilig wird und sie unterschiedliche Fähigkeiten erproben können. Sie werden von einer Sozialarbeiterin betreut und gecoacht.

Die WerkStadt Bregenz ist ein wichtiges und erfolgreiches Jugendbeschäftigungsprojekt. Durch die gute Abstimmung von Sozialarbeit und Arbeitsabwicklung sowie durch klare Vernetzungsstrategien konnte im vergangenen Jahr die Vielfalt der Aufgaben aus der Sicht aller Beteiligten positiv bewältigt werden. 


\section{Stadt Graz}

\section{Modellregion e-mobility Graz}

Elektromobilität als Säule eines nachhaltigen integrierten Mobilitätssystems im Großraum Graz.

Der Hype um Elektromobilität ist andauern - die Erwartungshaltung ist schwer zu erfüllen Lieferengpässe bei Fahrzeugen, Technologieentwicklung der Batterien, der Ladeinfrastruktur, rechtliche Rahmenbedingungen von Baugesetzen bremsen die Euphorie in der Umsetzung; die Kunden sind preissensibel und akzeptieren die hohen Preise nicht der Fahrspaß ist für jeden garantiert.

$\mathrm{Zu}$ den besonderen Umsetzungserfolgen gehört der Verleih von Elektrofahrzeugen als Flughafenshuttle mit Hertz - one way für 2 Stunden um 9,90 €, für Jahreskartenbesitzer um $6,90 €$. Einsatz von e-FZ im Firmenfuhrpark seit mehr als 6 Monaten ohne Probleme.

Als Umsetzungshemmnis zeigt sich, dass die öffentliche Ladeinfrastruktur nur langsam realisierbar ist; die gesetzlichen Rahmenbedingungen ergeben Unsicherheiten beim Betrieb von Ladesäulen; das Mobilitätsverhalten ist nur langsam zu ändern und muss gelernt werden.

Es ist geplant das Projekt Modellregion Großraum Graz in Verbindung mit dem ÖV weiter umzusetzen.

\section{Smart City / Zero Emission}

Im Rahmen des Vorprojektes Smart City Graz „I live Graz" werden nachhaltige Wirkungszusammenhänge etwa für energie- und verkehrsoptimierten Städtebau, optimierte Flächennutzung und BürgerInnen-Partizipation mit Stakeholdern und dem Projektkonsortium in Arbeitsgruppen untersucht. Dieser Erkenntnisse werden anschließend nach den Handlungsfeldern (Gebäude, Versorgung / Entsorgung, Mobilität, Information und Kommunikation (sustainable governance) System Stadt) in integrierte Leitlinien übergeführt. In Roadmaps 2020 und 2050 und (adaptierten) Aktionsplänen bis 2020 werden konkrete Maßnahmen den Betrachtungsebenen (Region / Stadt, Stadtteil, Quartier und Projekt) zugeordnet, und Vorschläge zur Anpassung bestehender Strategien erarbeitet.

Ziel ist die richtungsweisende Umsetzung eines integrativen Planungsprozesses, um eine zukunftsfähige und nachhaltige Stadtentwicklung zu ermöglichen, welche den Erhalt bzw. die Verbesserung der Lebensqualität als übergeordnetes Ziel hat.

Als Umsetzungshemmnisse stellen sich rechtliche Hindernisse (Gesetze, Verordnungen, Richtlinien) dar, aber auch finanzielle Komponenten und vorhandene (konfligierende) politische Strategien (Energiepolitik, Raumordnung, Verkehrspolitik).

Die im Rahmen des Vorprojekts „I live Graz“ definierten nachhaltigen Entwicklungsstrategien (Visionsmatrix, Indikatoren, Roadmap) bilden die Basis für ein zu entwickelndes Smart City Leitprojekt und dienen für den Entwicklungsprozess als Qualitätssteuerungstool.

Über diese Ausrichtung wird die europäische Strategie (SET) verfolgt. Zielt ist es, ein Demonstrations-Projekt von internationalem Charakter mit internationalen Partnerschaften zu entwickeln. 


\section{Stadt Linz}

\section{Lehrlingsoffensive}

Das seit dem Jahr 2004 laufende Projekt „Lehrlingsoffensive“ bedarf grundsätzlich einer laufenden Evaluierung und Anpassung der Ausbildungs- und Betreuungsmaßnahmen, um den ständig steigenden Anforderungen gerecht zu werden.

Hinsichtlich der bereits erfolgreich laufenden Unterstützungsangebote wie z.B. intensiver Förderunterreicht zur Bewältigung der Anforderungen in der Berufsschule, Betreuung durch BerufsausbildungsassistentInnen, spezielle Fachausbildungen und diverse sozialpädagogische Maßnahmen, ist eine Fortsetzung dieser Projekte geplant. Zusätzlich ist jedoch in Zukunft vor allem die Ausweitung spezieller psychosozialer Maßnahmen notwendig und geplant, welche Möglichkeiten eröffnen, rechtzeitig auf die persönlichen Bedürfnisse und Probleme der Jugendlichen zu reagieren.

In diesem Zusammenhang hat der Magistrat der Stadt Linz im Jahr 2011 die integrative Berufsausbildung hinsichtlich der Zufriedenheit der Lehrlinge, in Kooperation mit der Fachhochschule Linz im Detail analysiert. In der ein-semestrigen Lehrveranstaltung "Case Study Innovation“ von 9 Studierenden des Master-Studienganges "Service of General Interest", an der Fakultät für Gesundheit und Soziales in Linz, wurde die Lehrlingsausbildung der Stadt Linz evaluiert.

Folgende Untersuchungsgruppen wurden in die Evaluierung miteinbezogen:

- Lehrlinge der Stadt Linz, welche im Rahmen einer integrativen Berufsausbildung beschäftigt sind

- AusbilderInnen aus verschiedenen Berufsgruppen, welche entsprechend hohe Erfahrungswerte in der Ausbildung mit beeinträchtigten Jugendlichen vorweisen können

- BerufsausbildungsassistentInnen, welche für die Begleitung und Betreuung der Magistratslehrlinge zuständig sind

Die aus der Untersuchung gewonnen Ergebnisse konnten in verschiedenen Teilbereichen wie z.B. in der Ausweitung der psychosozialen Betreuung von Jugendlichen und Ausbilderlnnen, in der Installation einer Austauschplattform und in Teilbereichen zur Unterstützung und Steigerung der Arbeitszufriedenheit bereits umgesetzt werden.

Vor allem im Bereich der integrativen Berufsausbildung konnten in den letzten Jahren sehr positive Erfolge erzielt werden. Jugendliche mit speziellen Bedürfnissen und entsprechend hohem Betreuungsbedarf, konnten durch die intensive Unterstützung und gezielte Förderung im gewählten Lehrberuf mit sehr guten Erfolgen abschließen. Zusätzlich konnte durch verschiedenste Maßnahmen die Quote der vorzeiten Lehrvertragsauflösungen sehr gering gehalten werden und ein Großteil der Jugendlichen kann im Rahmen seiner Ausbildung die Berufsausbildung bei der Stadt Linz positiv beenden.

Besondere Startschwierigkeiten ergaben sich durch:

- Schwierigkeiten in der Erlernung der Ausbildungsinhalte durch negativen oder fehlenden Pflichtschulabschluss, sonderpädagogischen Förderbedarf bzw. Unterreicht nach Lehrplan einer Sonderschule

- Eingliederungsschwierigkeiten durch physische Beeinträchtigungen, wie z.B. Behinderung, Misshandlung, Drogenkonsum, problematische Familienverhältnisse usw.

- Sprachschwierigkeiten durch fehlende Deutschkenntnisse

- Demotivation und Integrationsprobleme durch jahrelange erfolglose Lehrstellensuche 
Es ist beabsichtigt, dieses Projekt beizubehalten und das Seminarangebot bzw. das Angebot des Förderunterrichts auszubauen. Interne Lehrmaterialien sollen erstellt bzw. weiterentwickelt werden. Die psychosoziale Betreuung soll ausgeweitet werden und eine laufende Evaluierung und Anpassung der Unterstützungsmaßnahmen ist vorgesehen. 


\title{
Euro-Plus-Pakt
}

\author{
Österreichische Maßnahmen 2012 \\ Übersicht
}

\section{Förderung der Wettbewerbsfähigkeit}

Als zentrales Element ist hier die Strategie für Forschung, Technologie und Innovation (FTI) anzuführen. Wichtige Schritte waren im Berichtszeitraum insbesondere die Einrichtung der Task Force FTI, die Erweiterung der Förderung im Rahmen des FWF auf indirekte Kosten, die Erhöhung der Forschungsprämie und die Erhöhung des Deckels für Auftragsforschung.

Im aktuellen Reformpaket (1. Stabilitätsgesetz 2012) sind hinsichtlich der Forschungsförderung durch die Forschungsprämie folgende Maßnahmen vorgesehen:

Anhebung des Forschungsdeckels bei der Auftragsforschung: Der bisherige Deckel in Höhe von $100.000 €$ bei den in Auftrag gegebenen Forschungsaufwendungen wird auf 1 Mio. $€$ angehoben, d.h. die maximale Forschungsprämie beträgt statt bisher $10.000 €$ nunmehr $100.000 €$. Die Neuregelung gilt für Prämien für Wirtschaftsjahre, die ab 1.1.2012 beginnen. Einbeziehung der Forschungsförderungsgesellschaft mbH (FFG) bei der jahresbezogenen Geltendmachung einer Prämie: Zur Verbesserung der Inhaltsprüfung soll anlässlich der Geltendmachung einer Prämie für eigenbetriebliche Forschung ein Gutachten der FFG beizubringen sein, welches das Vorliegen der Voraussetzungen für eine Prämie dem Grunde nach beurteilt. Dieses Gutachten dient dem Finanzamt als Entscheidungsgrundlage im Prämienverfahren.

Erhöhte Rechtssicherheit durch Forschungsbestätigung und Feststellungsbescheid: Forschungsbestätigung: Um dem Steuerpflichtigen insbesondere bei mehrjährigen Forschungsprojekten erhöhte Rechtssicherheit in Bezug auf die jährlich geltend zu machende Forschungsprämie zu geben, wird die Möglichkeit geschaffen, vom Finanzamt eine bescheidmäßige Bestätigung darüber zu erhalten, dass ein bestimmtes Forschungsprojekt die Voraussetzungen für eine Prämie dem Grunde nach erfüllt Forschungsbestätigung gemäß § 118a BAO). Dazu bedarf es ebenfalls eines Gutachtens der FFG, welches das Vorliegen der Voraussetzungen einer Forschung und experimentellen Entwicklung bestätigt.

Feststellungsbescheid über die jährliche Bemessungsgrundlage: ein Steuerpflichtiger kann sich vom Finanzamt die Richtigkeit der Bemessungsgrundlage für die Prämie bestätigen lassen. Dazu bedarf es einer diesbezüglichen Bestätigung des Wirtschaftsprüfers. Folgt das Finanzamt der Bestätigung, erlässt es einen - auf das jeweilige Wirtschaftsjahr bezogene Feststellungsbescheid über die Höhe der Bemessungsgrundlag für die Forschungsprämie (gemäß § 108c ABs- 8 EStG). Forschungsbestätigungen und Feststellungsbescheide können ab dem 1. Jänner 2013 beantragt werden.

Bereits mit Budgetbegleitgesetz 2011 wurde die Forschungsprämie nochmals von 8\% auf $10 \%$ erhöht.

Um die Rahmenbedingungen für exzellente Forschungsgruppen zu verbessern und die wettbewerbsorientierte Forschung an Universitäten zu stärken wurde der Fonds zur Förderung der wissenschaftlichen Forschung (FWF), Österreichs zentrale Einrichtung zur Förderung der Grundlagenforschung, ab 2011 in die Lage versetzt, 20\% der Overheadkosten auf alle vom FWF geförderten Einzelprojekte und auf Projekte des Programms zur Entwicklung und Erschließung der Künste (PEEK) zu finanzieren. 


\section{Österreichischer Hochschulplan}

Die übergeordnete Zielsetzung des Hochschulplans liegt darin, den österreichischen Hochschulraum besser abzustimmen und dadurch erfolgreich weiterzuentwickeln, höchste Qualität in Lehre und Forschung sicherzustellen und die internationale Sichtbarkeit zu erhöhen.

Teilprojekte des Hochschulplans:

Bauleitplan: Ziel ist eine klare und mit den Universitäten vor Ort abgestimmte Reihung künftiger Bauvorhaben und -notwendigkeiten, die dem vereinbarten Zeitplan gemäß abgearbeitet wird.

Forschungsinfrastruktur/Internationales: Mittels des Forschungsinfrastrukturplans wird bestimmt, wo künftig Großanlagen für die Grundlagenforschung errichtet werden. Ziel ist es, die internationale Wettbewerbsfähigkeit durch nationale Koordination und eine stärkere Profilierung durch Prioritätensetzung zu erreichen.

Koordinationsmaßnahmen: Ziele sind:

Entwicklung und Organisation eines institutionalisierten Koordinierungsgremiums („Hochschulkonferenz") und entsprechender Mechanismen unter Involvierung relevanter Stakeholder

Entwicklung von Koordinierungsmaßnahmen insbesondere auch für Lehre und Forschung Schaffung neuer Instrumente bzw. neuer Kommunikationskulturen zur Steuerung des Hochschulraums

Kapazitätsorientierte Studienplatzfinanzierung: Die Universitätsfinanzierung soll künftig auf die drei Säulen Lehre, Forschung bzw. Entwicklung und Erschließung der Künste sowie Infrastruktur aufgeteilt werden. Die "Studienplatzfinanzierung" ist als neue Universitätsfinanzierung zu sehen, die künftig insbesondere die Grundfinanzierung der Lehre der Universitäten sicherstellen soll. Dies bedingt $u$. a. eine Kapazitätenregelung unter Berücksichtigung einer Studierendenplatzzahlorientierung und ggf. einer Steigerung. Mit der schrittweisen Einführung der neuen Instrumente soll nach und nach ab der nächsten Leistungsvereinbarungsperiode (2013-2015) begonnen werden, so dass ein reibungsloser Übergang und eine Umstellung mit den Leistungsvereinbarungen ab 2015 möglich ist.

\section{Erhöhung der Anzahl der Fachhochschulplätze}

Die qualitative Weiterentwicklung des Fachhochschulsektors ist unter anderem im sogenannten Fachhochschulplan angeführt, der die inhaltlichen Schwerpunkte des FHSektors für die nächsten jeweils drei Jahre festlegt (2010/11 bis 2012/2013). Demnach sind unter anderem Schwerpunkte im Bereich Technik/Naturwissenschaften, Ausbau der berufsbegleitenden Studien, Ausbau der angewandten Forschung sowie Internationalisierung vorgesehen. Weiters wurden durch die Implementierung des Hochschul-Qualitätssicherungsgesetzes (HS-QSG) und der damit verbundenen Novellierung des Fachhochschul-Studiengesetzes (FHSTG) erweiterte Qualitätskriterien für den Fachhochschulsektor geschaffen. Durch die Fortführung der Offensivmittel werden am $\mathrm{FH}$ Sektor im Vollausbau im Studienjahr 2016-2017 zusätzlich ca. 5.000 Studienplätze geschaffen.

\section{Verbesserung der forschungsgeleiteten Lehr- und Studiensituation}

Zur Stärkung der Lehre zur Erhöhung der Qualität in der Hochschulbildung bei gleichzeitiger Effizienzsteigerung wurden zusätzliche Offensivmittel den Hochschulen zur Verfügung gestellt. Besondere Schwerpunkte liegen abgesehen vom Ausbau des FH-Sektors bei der Verbesserung der Studiensituation in den sog. „Massenfächern“ und in der Stärkung der wichtigen MINT-Fächer (Mathematik, Informatik, Naturwissenschaften und Technik). bei der Neustrukturierung der Kooperation von Universitäten mit außeruniversitären Forschungseinrichtungen und dem Neustart der universitären „Overhead-Finanzierung“ im Rahmen der Forschungsfinanzierung durch den FWF. 


\section{Ausbau ganztägiger Schulformen}

2006 war die Ausgangslage ein Angebot von 62.700 Betreuungsplätzen. Im Schuljahr 2011/12 sind es bereits 105.000 Plätze. In zumindest einer Schule pro Bezirk wird schulische Tagesbetreuung angeboten. Insgesamt gibt es bereits an 25\% der Schulen der Primar- und Sekundarstufe I Betreuungsangebot. Im Jahr 2012/13 soll es schon eine Verdoppelung gegenüber 2006 auf rund 120.000 Plätze geben.

Bis 2015 werden jährlich 80 Mio. € Bildungs-Offensivmittel investiert. Ziel ist es, dort ein Angebot an Tagesbetreuung zu schaffen, wo eine entsprechende Nachfrage besteht. Dafür werden die derzeit bestehenden 105.000 Plätze auf 160.000 Plätze für die schulische Tagesbetreuung ausgeweitet. Inklusive der Hortplätze sollen ab 2015 insgesamt 210.000 Plätze angeboten werden, was eine Verdoppelung des Angebots darstellt. Im Parlament wurde das Paket zum Ausbau der Ganztägigen Schulformen beschlossen: Neben klassen-, schulstufen-, und schulübergreifenden Angeboten wird schulartenübergreifende Tagesbetreuung ermöglicht.

Schon ab 12 angemeldeten Schülerlnnen - falls keine schulübergreifende Form der Ganztagesbetreuung möglich ist - muss ein verpflichtendes Angebot zu schulischen Tagesbetreuung zustande kommen.

Für den Bereich der Freizeitpädagogik gibt es eine Ausbildung als „akademischer Freizeitpädagoge“ (2 Semester, berufsbegleitend) an Pädagogischen Hochschulen.

Mit den Bundesländern wurden 15a-Vereinbarungen abgeschlossen, mit denen die Zuteilung der Offensivmittel, die als Anschubfinanzierung und zum Teil für den Ausbau der Infrastruktur eingesetzt werden, geregelt wird. Dabei werden auch einheitliche Qualitätskriterien verankert. Ziel ist insbesondere auch eine verstärkte Kooperation mit Sport- und Kulturvereinen sowie Musikschulen.

\section{Förderung der Beschäftigung / Nationaler Job Plan}

Österreich unterstützt die verstärkten Anstrengungen zur Bekämpfung der Jugendarbeitslosigkeit auf EU-Ebene im Sinne einer europäischen Jugendinitiative, wie sie in der Mitteilung der Europäischen Kommission „Chancen für junge Menschen“, in der Erklärung der Staats- und Regierungschefs vom 30. Jänner 2012, im Gemeinsamen Beschäftigungsbericht und im Synthesebericht zur Umsetzung des Europäischen Semesters dargestellt werden ${ }^{41}$, und hat bereits mit den im Anhang II des Nationalen Reformprogramms 2011 genannten Euro-Plus-Pakt-Maßnahmen zur Beschäftigungsförderung einen deutlichen Schwerpunkt auf junge Menschen gesetzt.

Im nationalen Kontext wird der Arbeitsmarkt- und Beschäftigungspolitik für Jugendliche im Jahr 2012 erneut höchste Priorität eingeräumt. Im Jahr 2011 standen für die Förderung von Lehrverhältnissen, Beschäftigung und Qualifizierung Jugendlicher mit mehr als 600 Mio. $€$ aus Mitteln des Arbeitsmarktservice, des Insolvenz-Entgelt-Fonds und des

Bundessozialamtes zur Verfügung - damit sind Jugendliche die am intensivsten unterstützte Zielgruppe der österreichischen Arbeitsmarktpolitik. Der Fördermitteleinsatz bleibt auch 2012 auf stabilem Niveau.

Mit der Umsetzung der Maßnahme Jugendcoaching (siehe auch Bildungs- und Armutsziel) wird ab 2012 vorerst in den Bundesländern Wien und Steiermark ein Beratungs- und Betreuungsangebot zur Begleitung von ausgrenzungsgefährdeten SchülerInnen im 9. Schulbesuchsjahr etabliert. Der Vollausbau in allen Bundesländern ist stufenweise bis zum Jahr 2014 geplant. Ziel des Jugendcoaching ist es vor allem,

\footnotetext{
${ }^{41}$ Insbesondere die Verweise auf eine „Jugendgarantie“, mit der "gewährleistet werden soll, dass alle jungen Menschen innerhalb von vier Monaten nach Verlassen der Schule eine Arbeitsstelle oder einen Ausbildungs- oder Weiterbildungsplatz erhalten“ (KOM(2011) 933 endg, S. 11)
} 
- ausgrenzungs-und schulabbruchgefährdete Schülerlnnen zu beraten und zu begleiten, sodass der individuell richtige Bildungsweg gefunden wird,

- verstärkt Orientierung in Bezug auf die Bildungs- und Berufslaufbahn zu geben, auch für Jugendliche mit sonderpädagogischem Förderbedarf (die bis 2011 durch die Maßnahme Clearing betreut wurden),

- psychosoziale Probleme aufzufangen, die den Bildungsweg gefährden könnten,

- Weitervermittlung zu anderen Beratungsstellen bzw. auch zu Praktikumsplätzen.

Beim Erfolgsfaktor duale Berufsausbildung wurden in der betrieblichen Lehrausbildung neue Akzente zur Absicherung des individuellen Ausbildungserfolgs gesetzt. Das mit der Novelle zum Berufsausbildungsgesetz 2011 verankerte Lehrlingscoaching ermöglicht ab 2012 den Aufbau eines Beratungs-, Begleitungs- und Unterstützungsangebots für Lehrlinge und Unternehmen u.a. zur Vermeidung von Lehrabbrüchen. Dabei sollen basierend auf den positiven Erfahrungen mit der Berufsausbildungsassistenz Informationen bereitgestellt, regelmäßig Kontakt zu Lehrling, Betrieb und Berufsschule gehalten und die Qualitätssicherung gefördert werden.

Die bereits im Nationalen Reformprogramm 2011 beschriebenen Maßnahmen „Aktion Zukunft Jugend" und Ausbildungsgarantie ${ }^{42}$ werden weitergeführt. Die Umsetzung der Überbetrieblichen Lehrausbildung (ÜBA) erfolgt auch im Ausbildungsjahr 2011/2012 auf hohem Niveau. Damit wird jenen Jugendlichen, die keine betriebliche Lehrausbildung absolvieren können, die Chance auf eine hochwertige Fachausbildung eröffnet. Eine Evaluierung der arbeitsmarktpolitischen Effekte der überbetrieblichen Lehrausbildung bestätigt, dass die ÜBA einen nachhaltigen Beitrag zur Verbesserung der Arbeitsmarktsituation jener Jugendlichen leistet, die durch sie einen Lehrabschluss erreichen.

Die Unterstützungsinstrumente für die Lehrlingsausbildung in den Betrieben sollen mit folgenden Maßnahmen weiterentwickelt werden:

- Gestaltung der betrieblichen Lehrstellenförderung entsprechend den Ausbildungserfordernissen der Unternehmen (laufender Prozess)

- Einheitliche Standards bei der Lehrabschlussprüfung durch Überprüfung von Prüfungsbeispielen durch „Clearingstelle“ (ab 2012)

- Praxisrelevante Ausbildungsmaterialien für Ausbilder, ergänzend zu den Berufsbildern (ab 2012)

Folgende Maßnahmen werden in der außerschulischen Jugendförderung fortgeführt: Bundes-Jugendförderung: Schwerpunktförderung Berufsorientierung Jobtalks 2.0

Die Jugend-Implacementstiftung „Just NEU“ sowie die zwanzig Produktionsschulen ${ }^{43}$ werden auch im Jahr 2012 fortgeführt.

Im Sinne einer zukunftsorientierten Integrationspolitik sieht der Nationale Aktionsplan für Integration die Forcierung der Arbeitsmarktintegration von Jugendlichen mit

Migrationshintergrund vor. Junge MigrantInnen, insbesondere bildungsferne Jugendliche, sollen verstärkt in Qualifizierungsmaßnahmen für Berufe einbezogen werden, für die am Arbeitsmarkt eine starke Nachfrage besteht. Eine gerade im Hinblick auf die Zielgruppe der jugendlichen MigrantInnen wichtige Rolle spielen die zur Unterstützung des Berufseinstiegs auf- und ausgebauten Programme wie die Förderung der überbetrieblichen Lehrausbildung oder niederschwelligere Angebote in diesem Bereich wie die „Produktionsschulen“.

Profitieren sollen Jugendliche mit Migrationshintergrund darüber hinaus aber auch von den

\footnotetext{
${ }^{42}$ Siehe auch Maßnahmenübersicht Nationales Reformprogramm 2011

${ }^{43}$ Siehe auch Maßnahmenübersicht Nationales Reformprogramm 2011
} 
zur Dropout-Vermeidung eingesetzten Maßnahmenprogrammen des Jugend- und Lehrlingscoachings.

\section{Verbesserung der langfristigen Tragfähigkeit der öffentlichen Finanzen}

Im Zusammenhang mit der Sicherstellung der langfristigen Finanzierbarkeit des öffentlichen Pensionssystems wird eine Reihe von Maßnahmen zur Erhöhung des effektiven Pensionsantrittsalters durchgeführt.

- Weiterführung der Reform des Invaliditätspensionsrechtes: Ausbau der gesundheitlichen und beruflichen Rehabilitation;

- Stufenweise Anhebung des für den Tätigkeitsschutz (Schutzbestimmung für die Inanspruchnahme einer Invaliditätspension) maßgeblichen Alters vom 57. auf das 60. Lebensjahr.

Das Stabilitätspaket 2012-2016 der Bundesregierung zielt auch darauf ab, bis zu 195.000 gesundheitlich beeinträchtigte und ältere Menschen länger im Erwerbsleben zu halten. Es setzt auf die Erhöhung des faktischen Pensionsantrittsalters durch

- Fortführung der Reform der Invaliditätspension - Ausweitung von beruflicher Rehabilitation $^{44}$ (siehe auch Armutsziel),

- Verschärfung der Anspruchsvoraussetzungen für die Korridorpension (Pensionsantritt ab dem 62. Lebensjahr mit jährlichen Abschlägen) - diese Pensionsart kann künftig erst nach 40 anstatt von 37,5 Versicherungsjahren in Anspruch genommen werden,

- Erhöhung des Abschlags pro Jahr des vorzeitigen Pensionsantritts (d.h. vor dem Regelpensionsantrittsalter) von $4,2 \%$ auf $5,1 \%$.

Weitere Anreize zu einem längeren Verbleib im Erwerbsleben soll ab 2014 das so genannte Kontogutschriftmodell setzen. Damit wird ein einheitliches Pensionskonto für alle Versicherten geschaffen. Somit ist die Pensionsberechnung klar nachvollziehbar und der Pensionsanspruch aus der Kontomitteilung ablesbar.

Im Bereich Arbeitsmarkt ist mit dem Stabilitätsgesetz 2012 beabsichtigt, die Inanspruchnahme des Altersteilzeitgeldes nicht nur (wie derzeit) bis zur frühestmöglichen Erfüllung der Anspruchsvoraussetzungen für eine Alterspension, sondern darüber hinaus bis zum gesetzlichen Pensionsalter (max. fünf Jahre) zu ermöglichen. Gleichzeitig mit der für Jänner 2013 geplanten Änderung soll die geblockte Variante der Altersteilzeit nur mehr bei Einstellung einer Ersatzkraft (einer vormals arbeitslosen Person oder eines Lehrlings) möglich sein.

Geplant ist auch, zur Finanzierung von Förderangeboten die ArbeitslosenversicherungsBeitragspflicht bis 63 Jahre bzw. bis zum Erreichen des Pensionsanspruches auszuweiten. Durch Beschäftigungsförderung für ältere Arbeitslose sollen bis 2016 zusätzlich zu den bestehenden Eingliederungsbeihilfen 40.000 über 50-Jährige einen neuen Arbeitsplatz bekommen.

Rund 70.000 Personen sollen jährlich zusätzlich im Rahmen von „fit2work“ beraten werden, 45.000 ältere und gesundheitlich beeinträchtigte Personen, sollen zusätzlich in AMSFörderungen einbezogen werden und 32.000 stärker beeinträchtigte, aber noch arbeitsfähige

\footnotetext{
${ }^{44}$ Für Menschen unter 50 Jahren, die unter Berücksichtigung ihrer gesundheitlichen Entwicklung eine Chance auf Wiedereingliederung in den Arbeitsmarkt haben, sollen Invaliditätspensionen durch Leistungen des Arbeitsmarktservice ersetzt und eine Wiedereingliederung in den Arbeitsmarkt angestrebt werden. Sind die Voraussetzungen für eine Pension aus dem Versicherungsfall der geminderten Arbeitsfähigkeit zumindest „wahrscheinlich“ erfüllt, so besteht ein Rechtsanspruch auf berufliche Rehabilitation. Diese Neuregelungen werden im Laufe des Jahres 2012 verhandelt und abgeschlossen.
} 
Personen sollen von beruflichen Rehabilitationsmaßnahmen profitieren. In Summe ist geplant, für die Arbeitsmarktoffensive über $€ 750$ Mio. zur Verfügung zu stellen.

\section{Stärkung der Finanzstabilität}

Die straffe Umsetzung der Haushaltsrechtsreform in ihren zwei Schritten 2009 und 2013 hat bereits mit ihrer Hauptelementen wie dem bindenden mehrjährigen Ausgabenrahmen, Flexiklauseln und der Performance-Orientierung den Grundstein für die langfristige Nachhaltigkeit der öffentlichen Haushalte in Österreich gelegt. Der Beschluss der „Österreichischen Schuldenbremse“ im Parlament vom 7. Dezember 2011 führt diese stabilitätsorientierte Ausrichtung konsequent fort. Mit diesem Konzept wird der strukturelle Budgetsaldo zur neuen Zielgröße der Wirtschaftspolitik. Bei Abweichungen sind zudem rasch greifende Korrekturmechanismen vorgesehen. Dabei werden Abweichungen auf einem Kontrollkonto erfasst und müssen bei Überschreiten eines Schwellenwertes konjunkturgerecht zurückgeführt werden. Durch diesen Ansatz wird gewährleistet, dass Schulden in konjunkturellen Aufschwungsphasen abgebaut werden und die stabilisierende Funktion öffentlicher Finanzen im Konjunkturabschwung gewahrt wird. Zudem stellt eine präzisere Zuweisung von budgetärer Verantwortung an alle Regierungsebenen eine gesamtstaatliche Orientierung zur budgetären Zielsetzung sicher. Als weitere Stütze ist im Gesetzestext explizit festgeschrieben, dass Rechtsakte der Europäischen Union zur Einhaltung der Haushaltsdisziplin erfüllt werden müssen. Die Schuldenbremse entspricht mit einem vorgeschriebenen strukturellen Defizit des Bundes von maximal $-0,35 \%$ des BIP im Jahr 2017 einer ambitionierten Umsetzung der Vorgaben des europäischen Fiskalpakts.

Der Österreichische Stabilitätspakt (ÖStP) 2011 ist rückwirkend mit 1.1.2011 in Kraft getreten und hat u.a. ${ }^{45}$ auch eine „Rendez-vous-Klausel“ bei Änderung von EU-rechtlichen Vorgaben (Verhandlungen zur Anpassung) vorgesehen. Aufgrund der verstärkten wirtschaftlichen Governance der EU-Mitgliedstaaten ergibt sich bereits jetzt die Notwendigkeit, Verhandlungen zur Anpassung des ÖStP 2011, der bis 2014 abgeschlossen wurde, zu führen und durch strengere Ziele als bisher die Umsetzung des neuen Konsolidierungspfades und damit die Erreichung eines strukturell ausgeglichenen Haushaltes für ganz Österreich ab 2017 sicherzustellen. Der_Österreichische Stabilitätspakt ist insbesondere auch Anker für die Umsetzung einer „Schuldenbremse“ für Bund, Länder und Gemeinden. Mit den Ländern und Gemeinden wurde dazu am 29. November 2011 in Salzburg eine Einigung über wesentliche Inhalte erzielt, die nun im Detail ausverhandelt werden.

\footnotetext{
${ }^{45}$ Der ÖStP 2011 hat eine Reihe an Neuerungen gebracht: ambitionierte, realistische Stabilitätsbeiträge von Bund, Ländern und Gemeinden, Verschärfungen der Sanktionen bei Zielverfehlung, Verbesserungen der Haushaltskoordinierung und mittelfristigen Ausrichtung der Haushaltsführung (die erste Datenlieferung auf Basis der neuen Formulare erfolgt erstmals im Juni 2012), die Festlegung von autonomen Haftungsobergrenzen für Bund, Länder und Gemeinden, erhöhte Transparenz und eine „Rendez-vous-Klausel“ bei Änderung von EU-rechtlichen Vorgaben (Verhandlungen zur Anpassung).
} 